\title{
Quantifying the Diffusion of Membrane Proteins and Peptides
}

\author{
Dissertation \\ for the award of the degree \\ "Doctor rerum naturalium" \\ of the Georg-August-Universität Göttingen \\ within the doctoral program GGNB \\ of the Georg-August University School of Science (GAUSS) \\ submitted by \\ Kerstin Weißs \\ from Frechen
}

Göttingen 10.06.2013 

Members of the Thesis Committee:

\section{Prof. Dr. J. Enderlein (Reviewer)}

3rd Institute of Physics - Biophysics, Faculty of Physics, Georg-August-University Göttingen

\section{Prof. Dr. S. Köster (Reviewer)}

Institute for X-Ray Physics, Faculty of Physics, Georg-August-University Göttingen

\section{Prof. Dr. A. Janshoff}

Department Physical Chemistry, Faculty of Chemistry Georg-August-University Göttingen

Date of oral examination: 05.08 .2013 


\section{Affidavit}

Hereby, I declare that the presented thesis has been written independently and with no other sources and aids than quoted.

Parts of this thesis have been published in

K. Weiß, A. Neef, Q. Van, S. Kramer, I. Gregor, J. Enderlein, "Quantifying the Diffusion of Membrane Proteins and Peptrides in Lipid Membranes with 2-Focus Fluorescence Correlation Spectroscopy", Biophysical Journal, 2013, 105, 455-462.

and

K. Weiß, J. Enderlein, "Lipid Diffusion within Black Lipid Membranes with 2-Focus Fluorescence Correlation Spectroscopy", ChemPhysChem, 2012, 13, 990-1000.

Göttingen, 09.08.2013 
"You'll never find a rainbow if you're looking down."

(Charlie Chaplin) 


\section{Contents}

\begin{tabular}{ll}
\hline Abstract & 1
\end{tabular}

\begin{tabular}{lll}
\hline & Introduction & 3
\end{tabular}

1.1 Lipid Membranes . . . . . . . . . . . . . . . . . . . 3

1.1.1 Membrane Structure and Composition . . . . . . . . . . . 3

1.1 .2 Bilayer Model Systems . . . . . . . . . . . . . . . 6

1.2 Describing Membrane Diffusion - The Saffman-Delbrück Model . . . . 12

1.3 Diffusion Measurements in Membranes . . . . . . . . . . . . . . . . . 14

$\begin{array}{lll}2 & \text { Materials and Methods } & 17\end{array}$

2.1 Chemicals . . . . . . . . . . . . . . . . . 17

2.2 Black Lipid Membrane Preparation . . . . . . . . . . . . . 17

2.3 GUV preparation . . . . . . . . . . . . . . . . . . . . 18

$2.4 \quad$ Protein Expression, Purification and Labeling . . . . . . . . . . . . . 19

2.4 .1 Cytochrome B5 . . . . . . . . . . . . . 19

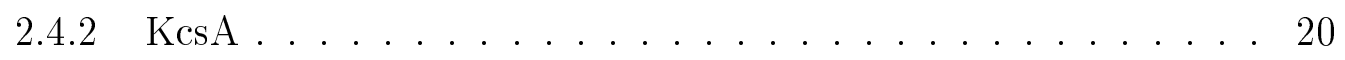

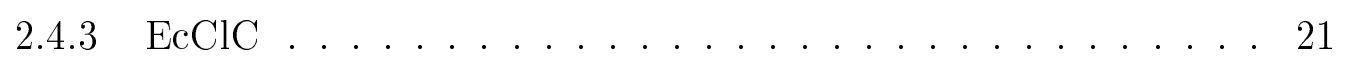

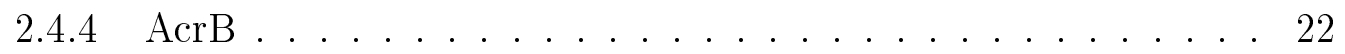

2.5 LUV Preparation and SNARE-mediated Vesicle Fusion . . . . . . . . 23

2.6 Electrophysiology . . . . . . . . . . . . . . . . . . . . 24

$2.7 \quad$ Dual-Focus Fluorescence Correlation Spectroscopy . . . . . . . . . . . 25

$2.7 .1 \quad$ Fluorescence . . . . . . . . . . . . . . . . . 25

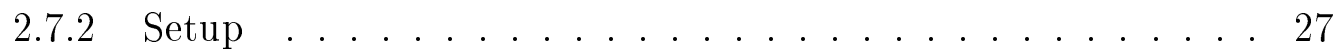

2.7.3 Time-Correlated Single Photon Counting (TCSPC) . . . . . . 29

2.7 .4 Theoretical Background . . . . . . . . . . . . . 31

2.7 .4 .1 2fFCS in Solution. . . . . . . . . . . . 31

2.7 .4 .2 2fFCS in Lipid Bilayers . . . . . . . . . . . 34

2.7.4.3 Performance of 2fFCS in Lipid Membrane Measurements ................... 36

$2.7 .5 \quad$ 2fFCS Measurements . . . . . . . . . . . . . . . 42

3 Lipid Diffusion in Black Lipid Membranes 45

$3.1 \quad$ Application of the Maximum Molecular Brightness Method for Focus Positioning on the BLM . . . . . . . . . . . . . 45

3.2 Influence of Solvent within Black Lipid Membranes . . . . . . . . . 47 
3.3 Influence of Mono- and Divalent Ions . . . . . . . . . . . . . 48

4 Membrane Protein Diffusion in Black Lipid Membranes 53

4.1 Protein Reconstitution via Direct Addition . . . . . . . . . . . . . . . 54

4.2 Protein Reconstitution via SNARE-mediated Vesicle Fusion . . . . 58

4.3 Influence of the Reconstitution Method on the Mechanism of Membrane Insertion . . . . . . . . . . . . . . . . . . 62

4.4 Models for describing Protein Diffusion in Membranes . . . . . . . . 63

5 Conclusions \& Outlook 69

\begin{tabular}{ll}
\hline Appendix & 73
\end{tabular}

Membrane Thickness Determination . . . . . . . . . . . . 73

Bibliography ............................ 76

List of Figures . . . . . . . . . . . . . . . . . . . . . 83

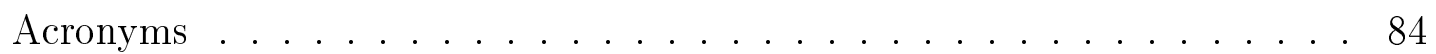

Acknowledgments . . . . . . . . . . . . . . . . 88 


\begin{abstract}
Lipid and protein diffusion in membranes is a fundamental requirement for many signaling processes in biological cells. Therefore, measuring protein and lipid mobility in lipid bilayers with high accuracy is essential for understanding biological mechanisms.
\end{abstract}

In 1975, Philip Saffman and Max Delbrück developed a model to describe protein diffusion in membranes. They predicted a logarithmic dependence of the protein's diffusion coefficient on its hydrodynamic radius $\left(D \propto \ln \frac{1}{R}\right)^{1}$. Recently, however, Gambin et al. observed a more Stokes-Einstein-like behavior, where the protein's diffusion coefficient and hydrodynamic radius are inversely proportional $\left(D \propto \frac{1}{R}\right)^{2}$. Previous theoretical and experimental studies reflect this discrepancy, illustrating the urgent need for accurate diffusion measurements in lipid bilayers.

To measure diffusion in membranes, Dual-focus Fluorescence Correlation Spectroscopy (2fFCS) was used. For correctly positioning the foci on the bilayer, a new method based on the maximum molecular brightness was developed, which is just as precise but much faster than the previously reported $z$-scan FCS ${ }^{3}$.

The maximum molecular brightness method was first applied to investigate lipid diffusion in Black Lipid Membranes (BLMs), in particular the influence of monoand divalent ions on neutral and charged lipid bilayers. It was found that monovalent ions do not influence lipid diffusion in neutral and charged BLMs. In contrast, divalent ions affect lipid diffusion differently depending on the lipid composition. While lipid diffusion in neutral membranes remained unchanged, lipid mobility in charged BLMs was decreased significantly upon addition of calcium ions. The reason for this is, that calcium can link two negatively charged head groups together thus increasing the membrane viscosity.

Secondly, protein diffusion in lipid bilayers was investigated in order to study the applicability of the Saffman-Delbrück model. Therefore, proteins of different sizes spanning one order of magnitude in radii were reconstituted into BLMs. The results revealed that, despite its simplicity, the Saffman-Delbrück model is suitable for describing protein diffusion in membranes. The Stokes-Einstein-like model, however, does not fit the obtained data all. A newer theoretical model developed by Petrov 
and Schwille ${ }^{4}$ was able to reproduce the Saffman-Delbrück results in the size range investigated.

In summary, BLMs in combination with 2fFCS provide a robust tool for investigating diffusion processes in lipid bilayers with high accuracy. For future studies, this system provides a great opportunity for investigating other membrane characteristics, such as membrane tension, which have been challenging to determine previously. 


\section{Introduction}

The lipid membrane is the interface between a cell and its environment. It consists of a lipid bilayer and a variety of embedded proteins which diffuse within the membrane and maintain essential functions of the cell such as ion transport and signaling. Diffusion processes within lipid membranes play a key role for the functional interaction of membrane-associated proteins. For example, many signal transduction cascades are initialized by ligand binding and subsequent oligomerization of activated receptors, both of which are diffusion-limited processes. Therefore, investigating lipid and protein diffusion in membranes is an essential aspect for understanding biological mechanisms. In this thesis, highly accurate measurements of lipid and protein diffusion in artificial model membranes are presented. The results allow for quantifying the influence of mono- and divalent ions on diffusion in different bilayer systems and for testing different models which describe protein diffusion in membranes.

\section{$1.1 \quad$ Lipid Membranes}

\subsubsection{Membrane Structure and Composition}

Studying structure and dynamics of lipid membranes has been an important part of biological, chemical and biophysical research during the last century. Membranes consist of a bilayer of lipids, an idea which was proposed for the first time in 1925 by Gorter and Grendel ${ }^{5}$ and was later, in 1935, replaced by the Davson-Danielli model which suggested that the lipid bilayer lies between two layers of globular proteins ${ }^{6}$. In 1975, Singer and Nicolson introduced the fluid mosaic model which supports the basic concept of a lipid bilayer. In this picture, the membrane is viewed as a twodimensional liquid into which proteins are incorporated ${ }^{77}$.

Lipids consist of a hydrophobic hydrocarbon tail and a hydrophilic head group. The hydrocarbon tails have different lengths and can be saturated or unsaturated. The headgroups determine the lipid class (e.g. phospholipid or sphingolipid) and specific functionality (e.g. phosphatidylcholine or phosphoethanolamine) and can be neutral or charged. Lipids form bilayers by self-assembly due to hydrophobic interactions of the tails. Bilayer formation allows burrying the hydrophobic parts of the molecules in the core while exposing the hydrophilic parts to the aqueous environment which 
is thermodynamically favorable. The hydrophobic core region of a bilayer usually has a thickness of around $3 \mathrm{~nm}$.

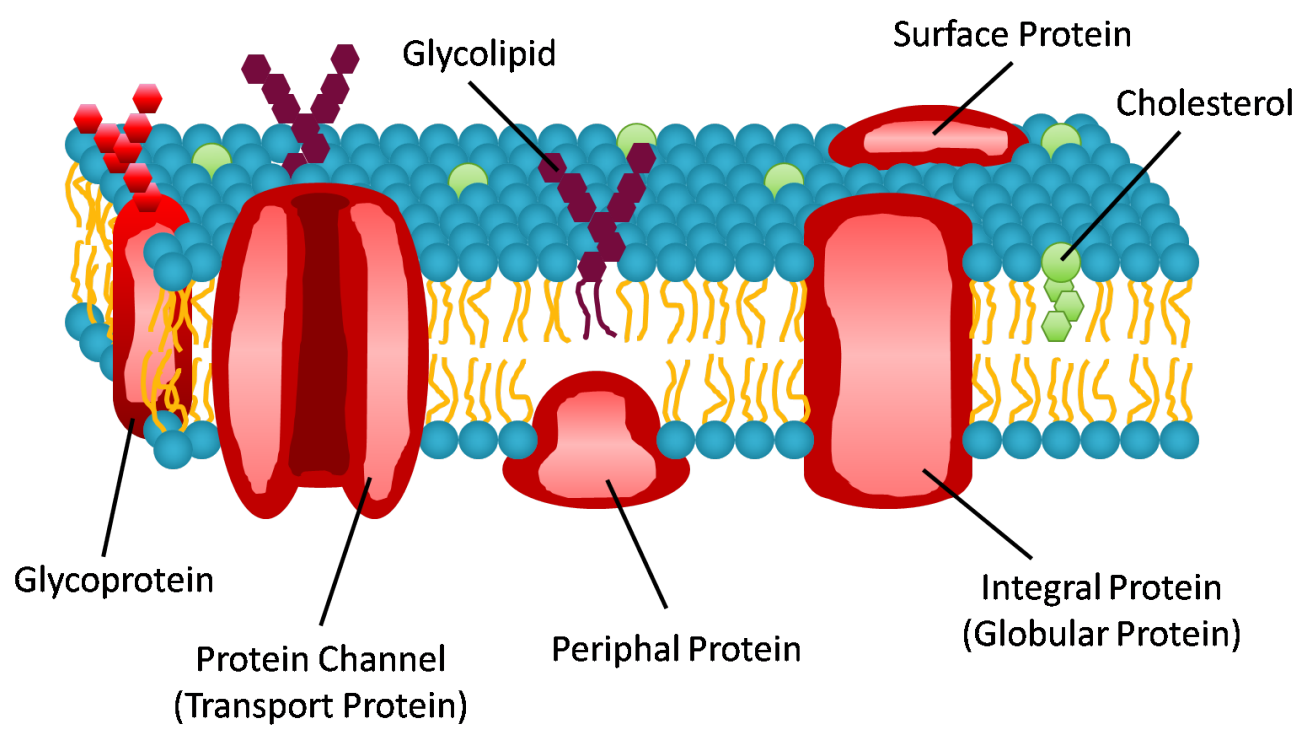

Figure 1: Biological Membrane. Proteins are incorporated into a lipid bilayer.

Naturally occuring membranes consist of phospholipids, sphingolipids and cholesterol and are mostly asymmetric which means that the lipid and protein compositions of the inner and outer leaflet of the bilayer can differ substantially. Due to this asymmetry, the membrane in a way defines the inside and outside of the cell. Proteins can be associated on either side of the membrane (peripheral membrane proteins) or pass through the bilayer with membrane-spanning segments (integral membrane proteins). The most common structure for these membrane-spanning segments is an $\alpha$-helix. It takes about 20 amino acid residues in an $\alpha$-helix to cover the $3 \mathrm{~nm}$ hydrophobic core of the bilayer.

Membranes are highly dynamic environments in which lipids and proteins diffuse rapidly and continuously in all lateral directions. The lateral mobility of the diffusing species within the bilayer is closely linked to the membrane fluidity, which depends on the temperature and the lipid composition. At low temperatures, the hydrocarbon tails become more ordered which leads to a closer packing (solid or gel phase). Upon temperature increase, the chains become increasingly disordered which in turn leads to an increase in fluidity. This phase transition from solid to liquid is characterized by the phase transition tempertaure $T_{m}$.

The fluidity is also influenced by the lipid composition. Bilayers containing high amounts of short-chained lipids with 16 carbon atoms or less also lead to an increase 
in fluidity as they will have less van-der-Waals interactions compared to longer chains. Moreover, chains containing cis double bonds increase the membrane fluidity since they cause packing defects between the saturated hydrocarbon chains.

Cholesterol has a special effect on fluidity, as it increases membrane viscosity if the membrane is very fluid to begin with or decreases the viscosity in case of membranes with low fluidity. This astonishing behavior can be explained with the size and structure of cholesterol (figure 2).

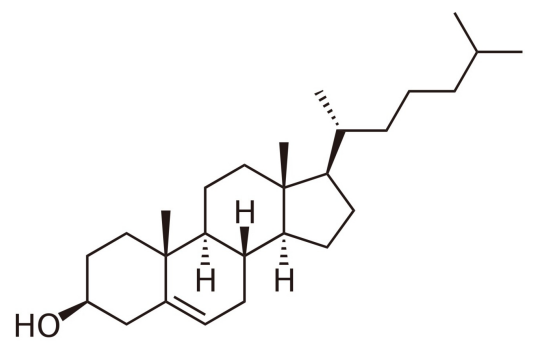

Figure 2: Chemical structure of cholesterol.

Membranes of low fluidity will consist of closely packed long-chain fatty acids. Cholesterol disturbs this tight packing leading to a fluidity increase. Membranes of high fluidity, however, will consist of lipids with unsaturated fatty acid chains containing cis double bonds. Cholesterol has the right size to pack into these defects caused by the cis double bonds which results in an increase of membrane viscosity. Thus, with respect to mobility, three different lipid phases can be distinguished: the solid (or gel) phase and upon melting the liquid-ordered $\left(\mathrm{L}_{\mathrm{O}}\right)$ and the liquid-disordered $\left(\mathrm{L}_{\mathrm{D}}\right)$ phase, which is shown in figure 3.

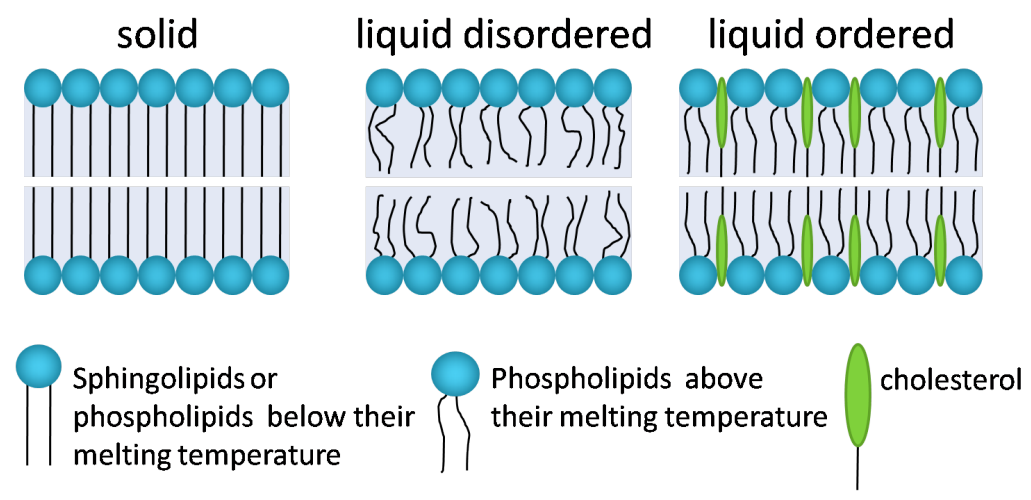

Figure 3: Lipid Phases. Depending on temperature and composition, the bilayer can be in the solid (gel) phase or upon melding in the liquid-ordered $\left(L_{O}\right)$ or liquid-disordered $\left(L_{D}\right)$ phase. Adapted from ref. $[8]$ 
In the solid phase, the lipids are tightly packed. The bilayer appears gel-like. Upon melting the fatty acid chains become increasingly disordered (liquid-disordered phase). Addition of cholesterol decreases the amount of defects compared to tightly packed chains and therefore leads to increased order and viscosity (liquid-ordered phase).

\subsubsection{Bilayer Model Systems}

Biological membranes are complex systems, which are highly crowded with membrane proteins. Membrane proteins occupy about $30 \%$ of the total surface area of the membrane ${ }^{9}$. Due to this complexity, simplified model systems with wellcharacterized lipid mixtures are used for in vitro studies. Three different model systems are commonly used for bilayer formation: Supported Lipid Bilayers (SLBS), Giant Unilamellar Vesicles (GUVs) and Black Lipid Membranes (BLMs).

Supported Lipid Bilayers 10 are artificial membranes which are either bound directly to a solid support via covalent or ionic bonds, or attachted to a polymer cushion on the surface 11 (figure 4).

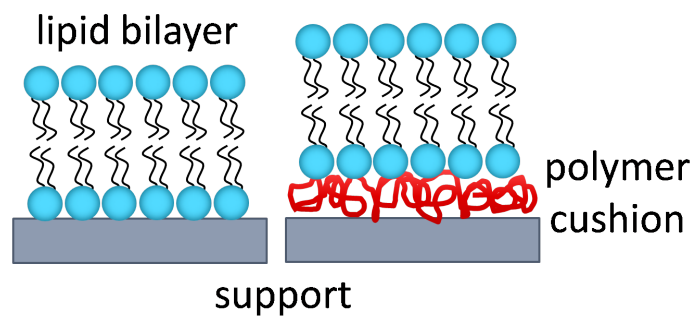

Figure 4: Schematic of Supported Lipid Bilayers (SLBs).

In most cases, mica or glass substrates are used for bilayer deposition. The membranes are formed using the Langmuir-Blodgett technique or vesicle speading. The resulting SLBs are highly stable over time and against mechanical stress. However, it was shown that the support influences the diffusion processes within the bilayer 12 ] making SLBs unsuitable for determining diffusion coefficients of free, undisturbed lipid membranes.

Giant Unilamellar Vesicles are lipid vesicles with diameters between $10-80 \mu \mathrm{m}$, although larger diameters of up to $200 \mu \mathrm{m}$ have also been reported when GUVs were prepared by microfluidic jetting ${ }^{13}$ (figure 5). Because of their size, GUVs can be considered to be locally planar. 


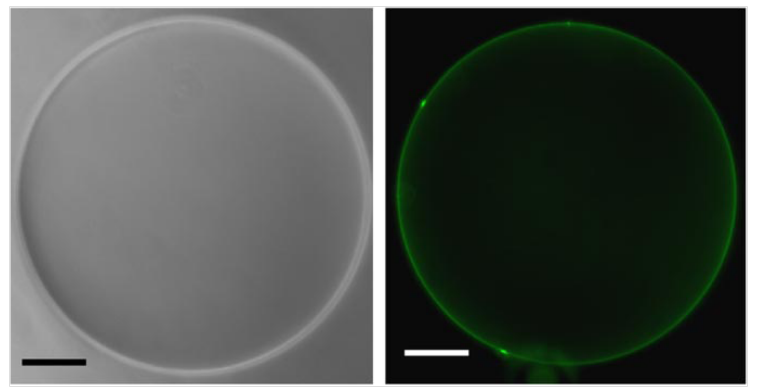

Figure 5: Giant Unilamellar Vesicles (GUVs) [13]. Left: Phase contrast image of the GUV. Right: Wide-field fluorescence image of the GUV labeled with BODIPY. Scale bar: $50 \mu \mathrm{m}$.

GUVs can be prepared by a variety of different methods. The most commonly used method is lipid film hydration, where a dry lipid film is deposited on a solid surface and vesicles form upon controlled hydration. This is schematically illustrated in figure 6 .

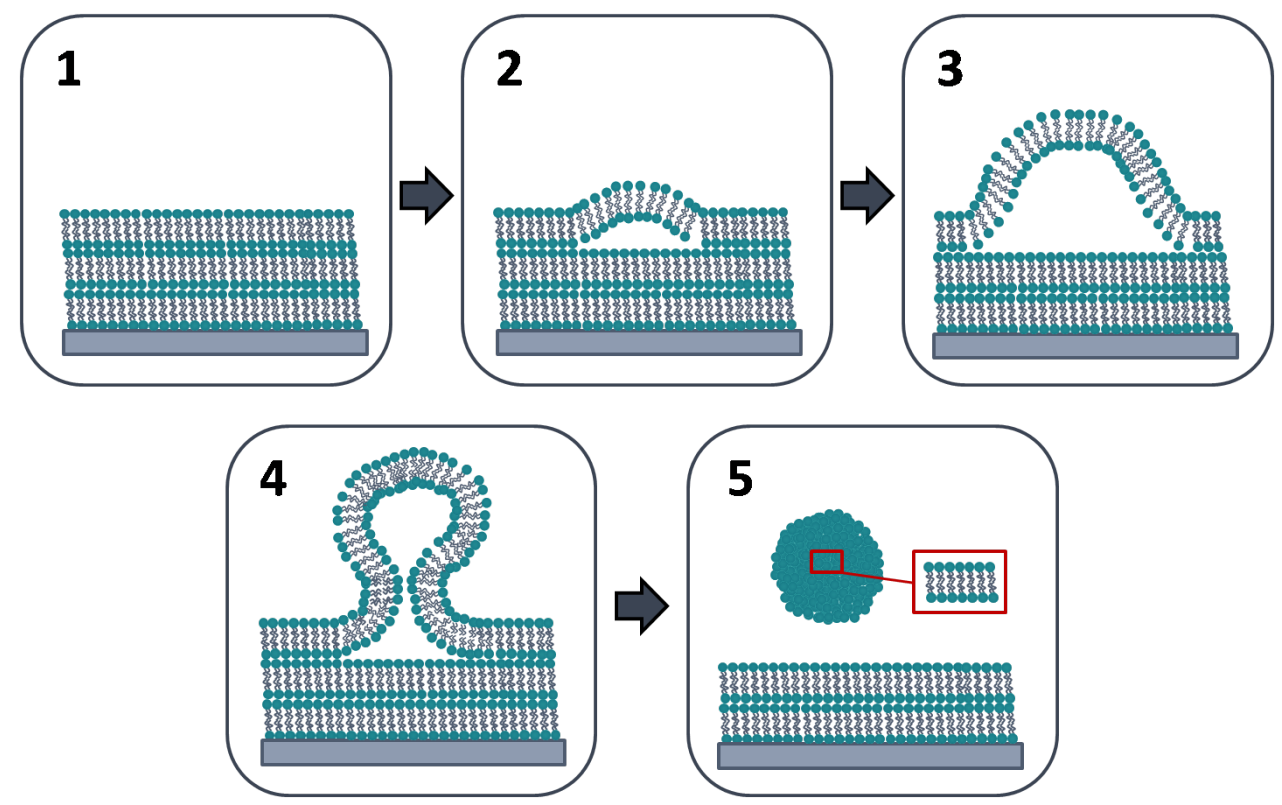

Figure 6: Formation of GUVs by lipid film hydration.

Depending on the lipid mixture, GUVs form either spontaneously (gentle hydration ${ }^{14}$ ) or upon application of an electric field (electroformation [15). Spontaneous vesicle formation is especially effective in the presence of charged lipids 16 . Moreover, it is important that the lipids are in the liquid-disordered phase upon hydration, i.e. the temperature has to be above the phase transition temperature $T_{m}$ which marks the main phase transition from solid-ordered to liquid-disordered phase.

Using Electroformation, GUVs consisting of neutral lipids can be prepared. In fact, 
the standard electroformation method ${ }^{15}$ does not yield vesicles if too many charged lipids are present 17 . Electroformation is usually performed at low ionic strength, although GUV formation at high ionic strength, i.e. physiological conditions, has also been reported 18 .

Alternatively, instead of generating GUVs from a dried lipid film, vesicles can be formed from a planar lipid bilayer. Therefore, a small volume of oil is added to an acrylic chamber with two compartments containing an acrylic divider (figure 7 A). One water droplet is deposited into the oil on each side of the chamber. Small Unilamellar Vesicles (SUVS) consisting of oil-insoluble lipids are then added into the water droplets. The SUVs consist of the lipids which will later form the GUV. The mechanism of GUV formation is illustrated in figure 7 .
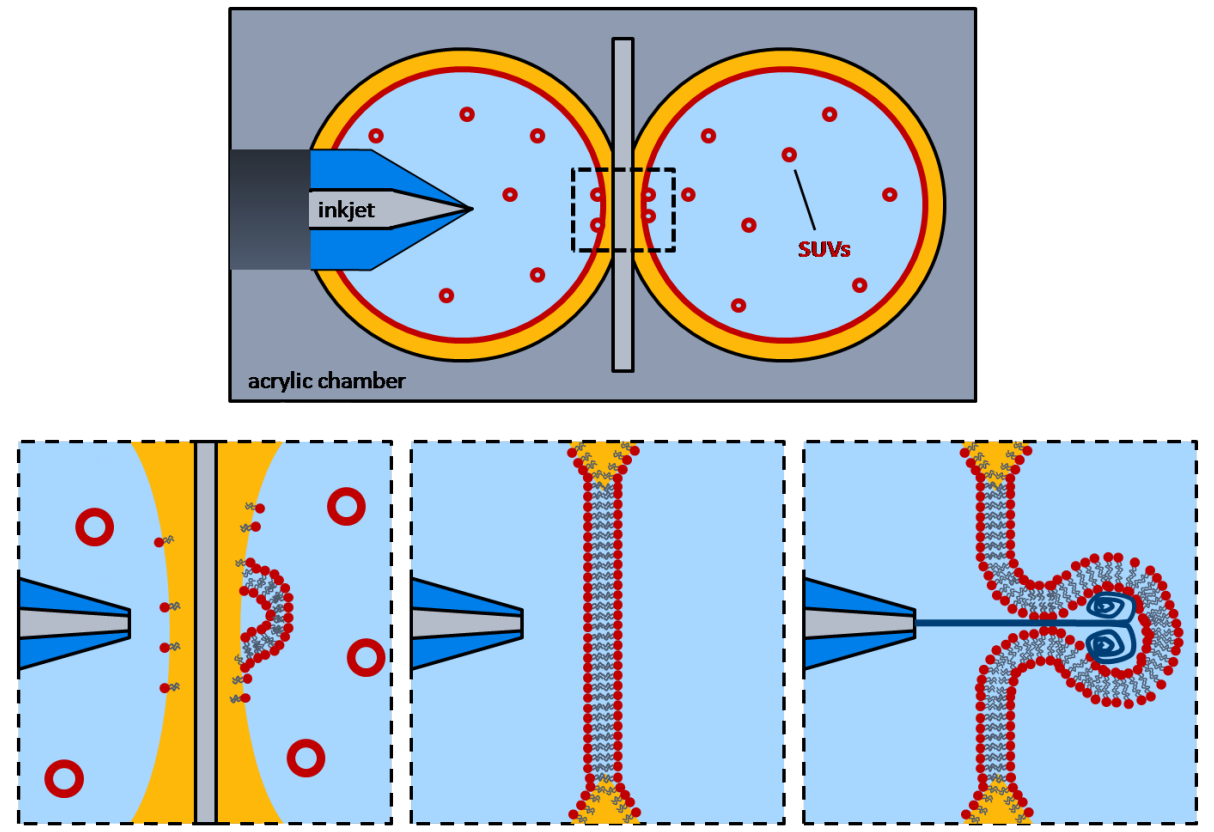

Figure 7: Microfluidic jetting for GUV preparation. Top: Acrylic chamber with piezoelectric inkjet and two aqueous droplets containing small unilamellar vesicles (SUVs) with oil-insoluble lipids (red). A divider separates the two aqueous droplets. Bottom: GUV formation. SUVs fuse at oil water interface and form continuous lipid monolayer around each droplet. Upon removal of the divider, a planar lipid bilayer is formed. GUVS are then formed by microfluidic jetting. 19$]$.

First, the SUVs gradually fuse to the oil-water interface of each droplet generating a continuous lipid monolayer along the interface. Upon removal of the divider, the monolayers come into contact forming a planar lipid bilayer. GUVs are then formed by microfluidic jetting. This process can be divided into three stages: initial 
membrane protrusion, membrane collapse and encapsulation, and finally separation of the vesicle from the bilayer. The diameter of the resulting GUV depends on the size of the initially generated membrane protrusion, which in turn is strongly affected by the convective and diffusive growth of the vortex ring structure generated by the jet 13 . The jetting method also allows for synthesizing asymmetric GUVs with different inner and outer leaflets by adding SUVs of different lipid composition into the two water droplets.

Overall, GUVs constitute solvent-free, free-standing bilayers. The drawback in using them, however, is that the solution inside the vesicles cannot be exchanged after synthesis. Moreoever, GUVs are very sensitive to osmotic pressure differences. Changes in the ionic strength of the surrounding medium with respect to the inside of the GUV can lead to membrane fluctuations or even rupture.

Black Lipid Membranes are lipid bilayers, which are spanned over a pore. The designation "black" lipid membrane refers to their behavior upon radiation with light. Due to their thickness of only 3-4 nm, light reflected off the front face of the membrane interferes destructively with light reflected from its back face. Therefore, it appears black in light microscopy.

BLMs are generated by the so-called painting technique. Therefore, lipids are first dissolved in an organic solvent. This solvent needs to be relatively viscous, which stabilizes the bilayer and prevents immediate rupture. Moreover, the solvent should have a low partition coefficient in water. Thus, decane or dodecane are commonly used for BLM preparation. The lipid solution is then applied with a brush, a syringe or via an automated pumping cycle across a small aperture. This aperture consists of a hydrophobic material, typically Teflon, and is surrounded by an aqueous buffer. The mechanism of BLM formation is shown in figure 8 .

Upon application of the lipid solution to the aperture, a lipid monolayer forms spontaneously at the interface between aqueous and organic phase on each side of the droplet. The hydrophobic walls of the aperture are wetted which leads to a thinning of the droplet in the center. The lipid monolayers thereby approach each other until they come close enough to fuse and from a lipid bilayer, leading to an exlusion of the remaining organic solvent in the center. On each side close to the aperture an annulus of solvent remains, which is required to stabilize the membrane. The annulus acts as a bridge between the membrane and the significantly larger Teflon sheet 20 . 

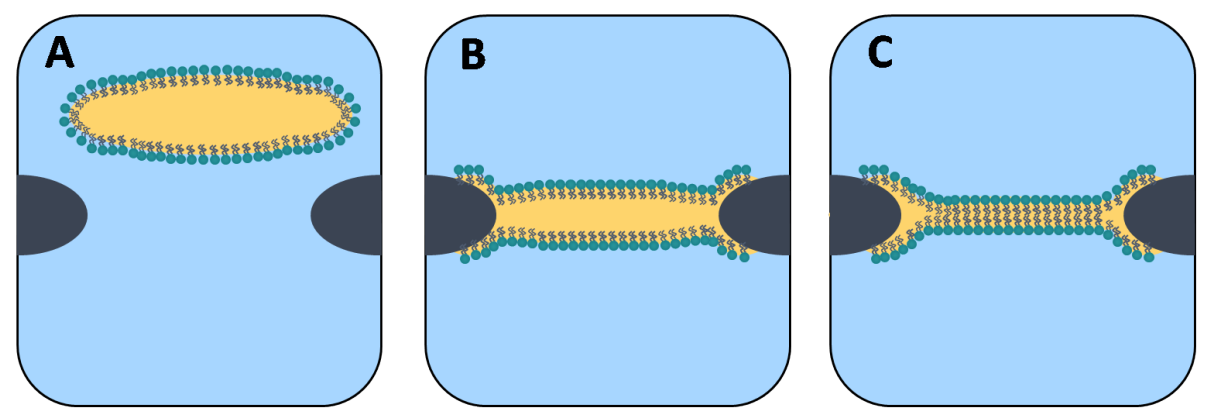

Figure 8: Black Lipid Membrane formation via painting. (a) Lipids are dissolved in an organic solvent and applied to the pore upon which a lipid monolayer is formed at the interface between aqueous and organic phase. (b) The droplet thins until the monolayers come close enough to form a bilayer. (c) The remaining solvent is excluded in the center and a solvent annulus is formed close to the pore rim which stabilizes the membrane.

Applying the painting method has the disadvantage, that small amounts of residual organic solvent could remain within the bilayer and thus influence diffusion processes within the membrane. To overcome this problem, solvent-free preparation strategies have been proposed. One option is to fuse two separate lipid monolayers on an airwater interface. Therefore, two water-containing compartments are separated by a wall while the pore, over which the membrane is spanned, is initially above the water surface (figure 9).

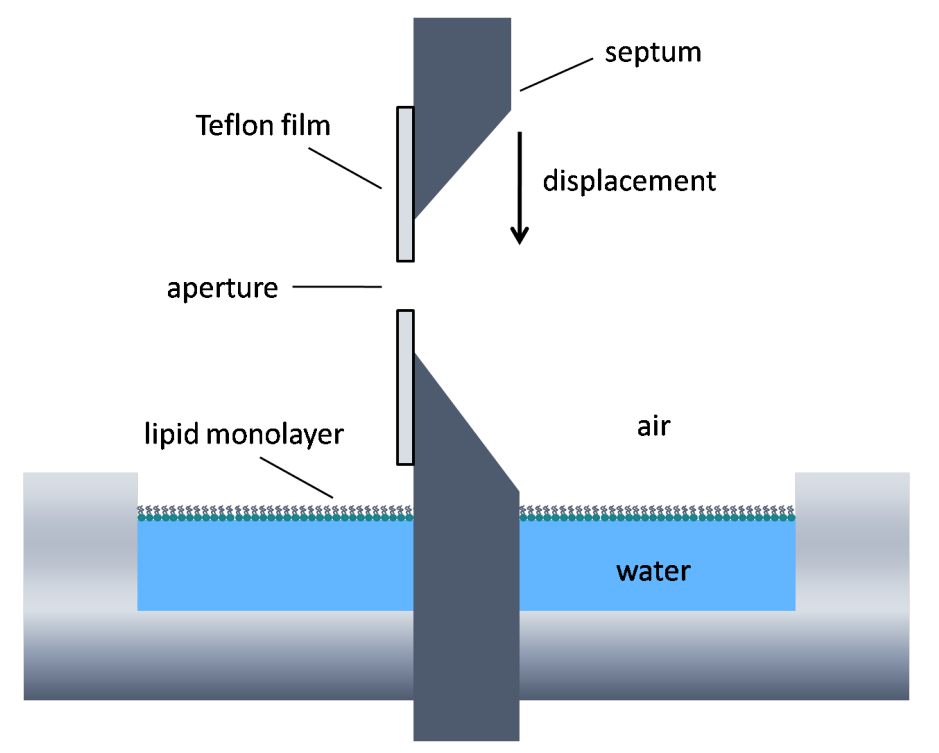

Figure 9: Preparation of a solvent-free BLM from a lipid monolayer at the air-water interface. [21]

The lipids are dissolved in a highly volatile organic solvent (e.g. chloroform). Upon addition to the water surface, the solvent evaporates which results in monolayer 
formation. The aperture is then lowered through the air-water interface thereby folding down the monolayers which leads to BLM formation across the pore 21 .

Alternatively, solvent-free pore-spanning membranes can be obtained by spreading GUVs over porous substrates (figure 10). These substrates consist of silicon nitride covered with a thin gold layer deposited on the surface, particularly on the pore rims. A mercaptoethanol solution is added to the substrate yielding a continuous self-assembled mercaptoethanol monolayer. This monolayer is the requirement for GUV deformation and rupture. When GUVs are added to the substrate, the vesicles adsorb onto the surface and deform. The increasing instability finally leads to GUV rupture resulting in the formation of a pore-spanning membrane-patch 22 . While the painting technique can also be applied to large apertures of up to $120 \mu \mathrm{m}$, the GUV speading technique is only applicable for small pore diameters $(\approx 1 \mu \mathrm{m})$.

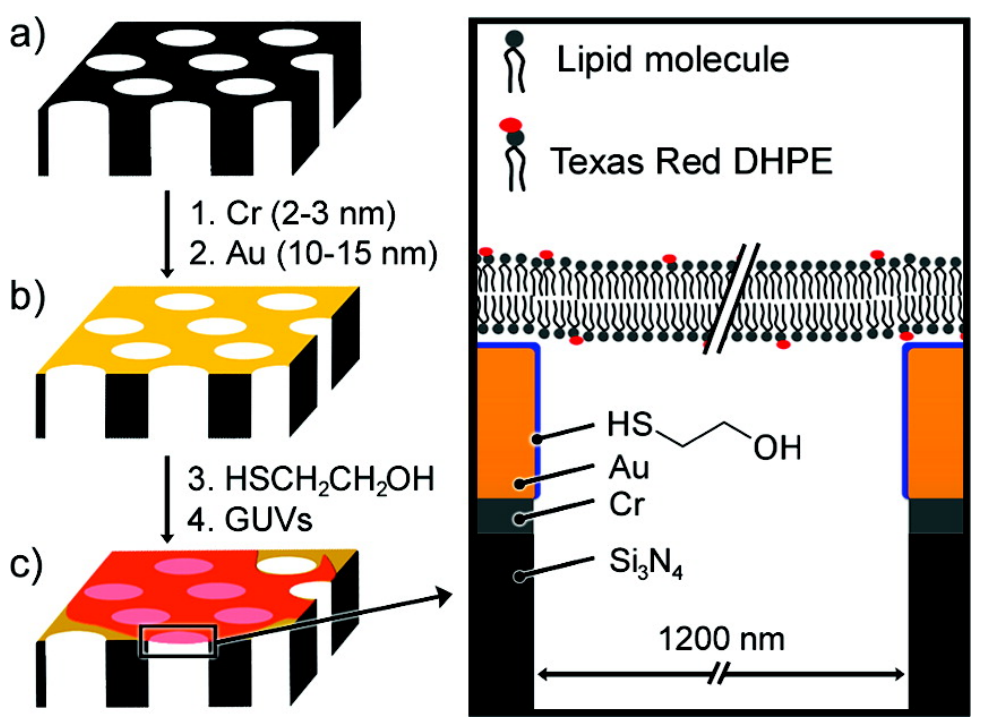

Figure 10: BLM formation upon spreading GUVs on a porous substrate. The substrate surface is first covered with a thin gold layer. Then a mercaptoethanol monolayer is deposited. Added GUVs adsorb to the surface and deform until they rupture which results in a pore-spanning bilayer patch. Figure taken from ref. 22].

The use of BLMs has several advantages. First, BLM formation and rupture can easily be monitored via capacitance measurements. Since BLMs separate two compartments which (in most cases) are easily accessible, electrodes can be placed on both sides of the bilayer. The bilayer has a large resistance and capacitance which allows for detecting membrane rupture as well as distinguishing the intact membrane from air bubbles or other impurities blocking the pore. Secondly, BLMs are rather stable towards high ionic strengths and differences in ion concentrations on both 
1. INTRODUCTION

sides of the bilayer. Since they are less prone to fluctuations compared to GUVs and constitute free-standing lipid bilayers, BLMs allow for highly accurate membrane diffusion measurements.

\subsection{Describing Membrane Diffusion - The Saffman-Delbrück Model}

Overall, biological membranes represent crowded environments in which lipids and proteins diffuse constantly. In 1975, Philip Saffman and Max Delbrück developed a model to describe protein diffusion in lipid bilayers ${ }^{11}$. In this model, proteins are viewed as cylinders of radius $R$ diffusing in a flat, two-dimensional membrane of height $h$ and viscosity $\mu_{m}$ surrounded by a solvent of viscosity $\mu_{s}$ (figure 11). They predicted a logarithmic dependence of the protein's diffusion coefficient $D$ on its hydrodynamic radius according to

$$
D_{S D}=\frac{k_{B} T}{4 \pi \mu_{m} h} \cdot\left(\ln \frac{1}{\epsilon}-\gamma\right)
$$

where $k_{B}$ denotes the Boltzmann constant, $T$ the temperature and $\gamma=0.5772$ is Euler's constant. The variable $\epsilon$ is defined as $\epsilon=\frac{\mu_{s} R}{\mu_{m} h}$. Recently, Gambin et al. challenged the Saffman-Delbrück model by observing a Stokes-Einstein-like behavior of the diffusion ${ }^{2}$ :

$$
D_{S E}=\frac{k_{B} T \lambda}{4 \pi \mu_{m} h \cdot R}
$$

All parameters have the same meanings as above, and $\lambda$ is a characteristic length introduced for dimensional reasons. This inverse linar dependence between the protein's diffusion coeffcient and radius differs substantially from the prediction of Saffman and Delbrück. In contrast, Ramadurai et al. ${ }^{23}$ found protein diffusion in membranes to comply with the Saffman-Delbrück model.

These contradictory experimental results have evoked an abundance of theoretical studies, which reflect the experimental situation by either justifying the StokesEinstein-like behavior or supporting the Saffman-Delbrück theory. Naji et al. present two explanations for the observed $D \propto 1 / R$ scaling. On the one hand, changes in bulk hydrodynamics due to a height mismatch between the membrane and the embedded protein could explain the findings. On the other hand, additional dissipative stresses due to lipid chain stretching, lipid tilt or local demixing in case of lipid mixtures could also yield the $D \propto 1 / R$ scaling. Overall, they argue that 


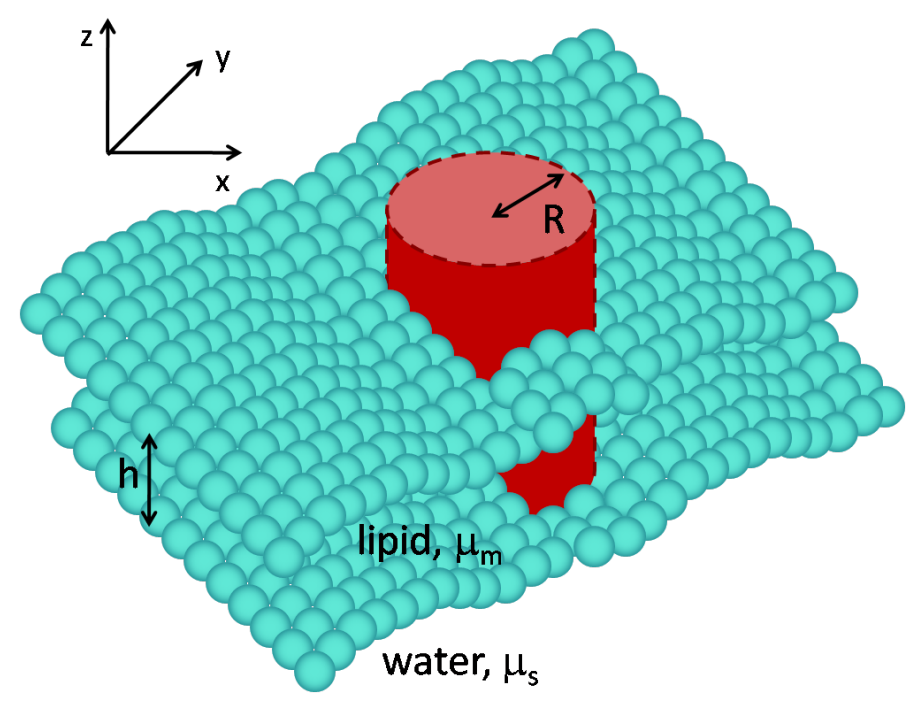

Figure 11: Saffman-Delbrück model. A cylindrical membrane inclusion is embedded into a lipid bilayer of viscosity $\mu_{m}$ and surrounded by an aqueous buffer of viscosity $\mu_{s}$. The inclusion moves laterally in the $x / y$ plane. Lipid tails have been omitted for clarity.

the Saffman-Delbrück model fails because the protein carries a deformed membrane patch changing its diffusive behavior ${ }^{24}$. Guigas and Weiss investigated the influence of hydrophobic mismatch on protein diffusion in more detail, arguing that the previous study by Naji et al. relied on ad hoc assumptions and might not be applicable to describe the diffusion of membrane inclusions with hydrophobic mismatch 25 . Guigas and Weiss' calculations showed that while hydrophobic mismatch changes the effective membrane viscosity, this does not alter the crude scaling behavior of protein diffusion. Therefore, the Saffman-Delbrück model should still be applicable even when accounting for mobility changes due to hydrophobic mismatch. The $D \propto 1 / R$ scaling emerges, according to their calculations, only for large membrane inclusions $\left(R_{c}>10 \mathrm{~nm}\right)$.

Addressing the problem that the Saffman-Delbrück model cannot be applied for larger membrane inclusions, Petrov and Schwille derived an analytical expression for the model suggested by Hughes, Pailthorpe, and White (HPW ${ }^{4}$ :

$$
D_{H P W}=\frac{k_{B} T}{4 \pi \mu_{m} h}\left[\frac{(2 \epsilon-1) \ln (\epsilon)-\gamma+\frac{8 \epsilon}{\pi}}{1+\frac{8 \epsilon^{3} \ln (\epsilon)}{\pi}+\frac{a_{1} \epsilon^{b_{1}}}{1+a_{2} \epsilon^{b_{2}}}}\right]
$$

The variables have the same meanings as above, and $a_{1}=0.433274, a_{2}=0.670045$, $b_{1}=2.74819$ and $b_{2}=0.614465$ are constants 25 . In the size range relevant for proteins, the HPW approximation mostly reproduces the Saffman-Delbrück result. The ambiguity in previous experimental and theoretical work reflects the urgent 
need for accurate quantitative data on protein diffusion in membranes.

\subsection{Diffusion Measurements in Membranes}

Several techniques have been used in order to measure lipid and protein diffusion processes in membranes, most of which are fluorescence-based. In Single Particle Tracking (SPT) ${ }^{26}$, molecules are either tagged with fluorescent dyes or with nonfluorescent colloidal gold or latex particles. Their movement is then tracked using video microscopy. The drawback, however, is that SPT yields relatively large errors, mostly caused by uncertainties and stochastic variabilities during data analysis, and that tagging with large beads can influence the diffusion.

In Fluorescence Recovery After Photobleaching (FRAP) ${ }^{27}$, fluorescently labeled molecules in a small area are bleached irreversibly by a short laser pulse of high intensity. Afterwards, fluorescently labeled molecules from the surrounding diffuse into the bleached region thereby restoring the fluorescence. The diffusion coeffcient is determined from the rate of fluorescence recovery. This method, however, requires high concentrations of fluorescently labeled molecules. Moreover, data evaluation is challenging. It requires precise knowledge about the size of the focus diameter which depends on manifold optical parameters and is therefore difficult to determine.

Alternatively, Fluorescence Correlation Spectroscopy ( $($ FCS $)$ can be employed to measure diffusion in membranes. FCS was first developed by Magde, Elson and Webb ${ }^{28}$ in 1972. It monitors small fluctuations in the fluorescence intensity caused by the movement of fluorophores into and ouf of the laser focus. These fluctuations are analyzed by calculating an autocorrelation function. If the concentration is sufficiently small so that on average only one molecule is within the focus, the decay of the correlation curve is characterized by the molecule's diffusion coefficient. Since FCS requires only low concentrations of fluorescently labeled molecules, it allows for working close to the limit of infinite dilution which is an advantage especially for biological samples which are difficult to prepare at high concentrations.

FCS, however, also has several drawbacks. Calculating the diffusion coeffcients requires precise knowledge about the size and shape of the focus which is rather difficult to obtain. Moreover, this requirement makes FCS sensitive to refractive index mismatch and cover slide thickness deviations. Calculating diffusion coefficients from FCS autocorrelation curves is only possible after calibration with a dye which has to be performed before each measurement. Furthermore, the calculated values for the diffusion coeffcients depend on the laser's excitation intensity due to optical 
saturation of fluorescence. For membrane diffusion measurements in particular, it needs to be taken into account that the diffusion processes are much slower than in solution. Therefore, longer measurement times are required for obtaining a sufficient number of trajectories for accurate evaluation, and special care must be taken to avoid photobleaching of the sample. Moreover, exact positioning of the focus on the membrane is essential since misalignment influences the results severely.

Originally, the correct focus position was determined by localizing the point of maximum fluorescence intensity. It was shown by Benda et al. ${ }^{3}$, however, that the maximum intensity does not necessarily coincide with the accurate focus position on the bilayer. Instead, they performed multiple FCS measurements along the optical axis perpendicular to the bilayer plane (z-scan FCS). The Particle Number ( $(\underline{\mathrm{PN}})$ and the diffusion time $\tau_{D}$ both depend on the focus diameter and therefore on the intersection between diverging laser beam and membrane. With increasing vertical distance between laser beam waist and bilayer, the observed diffusion time increases. The obtained dependence of the diffusion time on the vertical distance is fitted by a parabolic profile to determine the beam waist diameter and the relative position between laser focus and membrane, which is then used for calculating the lateral diffusion coefficient $D$. While $z$-scan FCS can be used to accurately determine the correct focus position on bilayers, recording multiple autocorrelation functions (ACFs) at different vertical focus positions is very time-consuming and makes this method highly sensitive to mechanical drift.

To circumvent these disadvantages, line-scan FCS ${ }^{29}$ or Dual-focus Fluorescence Correlation Spectroscopy (2fFCS) ${ }^{30]}$ can be used for measuring diffusion coefficients. Both methods apply the same basic idea of introducing an internal length-scale into the measurement by placing two detection regions at a well-known distance from each other. The ACFs of both detection volumes as well as their crosscorrelation are then calculated and used to determine diffusion coefficients.

In line-scan FCS, two parallel lines with known distance are scanned alternately. The resulting intensity traces are crosscorrelated yielding a crosscorrelation curve which reflects the diffusion of molecules from one detection volume to the other within the bilayer. Thus, the diffusion coefficient and beam waist $w_{0}$ can be obtained directly by fitting the correlation curves without additional calibration measurements 31 . The detection volumes are not stationary but moving which increases the statistical accuracy while decreasing the measurement time. Moreover, photobleaching is minimized because the residence time of molecules inside the detection volume is decreased. 
In 2fFCS, two overlapping foci are generated by a Nomarski prism. Precise knowledge of the focus distance, which depends only on the properties of the prism, provides an internal ruler to the system allowing for calculating absolute values for the diffusion coefficients and rendering 2fFCS a calibration-free method. Furthermore, 2fFCS is insensitive to cover slide thickness deviations and refractive index mismatch and is therefore much more robust and accurate than single focus FCS. In order to apply 2fFCS to diffusion measurements in membranes, a new method for positioning the foci was developed, which is described in detail in this thesis. It relies on localizing the maximum molecular brightness rather than performing multiple FCS measurements and therefore provides a fast and highly accurate tool for positioning.

To investigate diffusion processes in lipid bilayers, proteins covering one order of magnitude in hydrodynamic radii $(0.4 \mathrm{~nm}$ to about $4 \mathrm{~nm})$ were used. Much care has been taken to choose proteins which do not aggregate inside the membrane and are well-characterized in terms of crystal structure and functionality. The crystal structures for the respective proteins provide a measure for their radius, which is essential to investigate the applicability of the Saffman-Delbrück model. It needs to be noted, that there is a difference between the radius obtained from the respective crystal structures, the hydrodynamic radius and the cylindrical radius calculated by the Saffman-Delbrück model. However, these differences are sufficiently small so that they can be neglected with respect to the gross scaling behavior for sizedependent proteins diffusion in membranes.

The covered size range of proteins combined with the superior accuracy of $2 \mathrm{fFCS}$ and a highly stable BLM system therefore allows for obtaining precise diffusion data in lipid bilayers which will set a new benchmark for protein diffusion and lipid bilayer viscosity. 


\section{Materials and Methods}

\subsection{Chemicals}

1-palmitoyl-2-oleoyl-sn-glycero-3-phosphoethanolamine (POPE), 1-palmitoyl-2-oleoylsn-glycero-3-phosphocholine (POPC) , 1,2-di-(9Z-octadecenoyl)-sn-glycero-3-phospho(1'-rac-glycerol) sodium salt (DOPG) and 1,2-dioleoyl-sn-glycero-3-phosphoethanolamine-N-(biotinyl) sodium salt (Biotinyl-PE) were purchased from Avanti Polar Lipids (Alabaster, AL, USA). 1,2-dihexadecanoyl-sn-glycero-3-phosphoethanolamine $\left(\mathrm{DPPE}^{\text {Atto655 }}\right.$ was provided by Christian Eggeling (University of Oxford, UK). Dodecane, streptavidin and chloroform were obtained from Sigma Aldrich (Traufkirchen, Germany). Octyl glycoside ( $(\mathrm{OG})$ was purchased from Santa Cruz Biotechnology (Heidelberg, Germany). Decyl maltoside (DM) was obtained from Genaxxon Bioscience (Ulm, Germany). Alexa647 succinimidyl ester and Alexa647 maleimide were obtained from Life Technologies (Darmstadt, Germany).

\subsection{Black Lipid Membrane Preparation}

In order to prepare neutral lipid bilayers, POPC and POPE were dissolved in chloroform and mixed in a ratio of 40 weight\% to 60 weight\%, respectively. Negatively charged lipid bilayers were prepared by mixing the previously described POPC / POPE mixture with 1 weight\% DOPG in chloroform. The solvent was evaporated for 30 minutes and dodecane was added to the dry lipid mixture to yield a final lipid concentration of $10 \mathrm{mg} / \mathrm{mL}$. BLMs were then generated using a commercially available setup (Ionovation Bilayer Explorer, Ionovation GmbH, Osnabrück, Germany). Its main part is the Bilayer Slide (figure 12), which consists of a cover slide, a pore of $120 \mu \mathrm{m}$ diameter over which the membrane is spanned, and two perfusion channels, one above and one below the pore. These channels can be perfused individually, which allows for changing the buffer either in the entire chip or on only one side of the membrane after bilayer formation. The Bilayer Slide is designed in such a way, that the distance between pore and cover slide is sufficiently small to access the BLM with a high numerical aperture (N.A.) objective which allows for conducting 2fFCS diffusion measurements.

On the top, the Bilayer Slide contains openings for perfusion and lipid injection as well as two electrode ports. The electrodes reach into the upper and lower perfusion channel, respectively, and connect the chip to a patch clamp amplifier (EPC 10, 


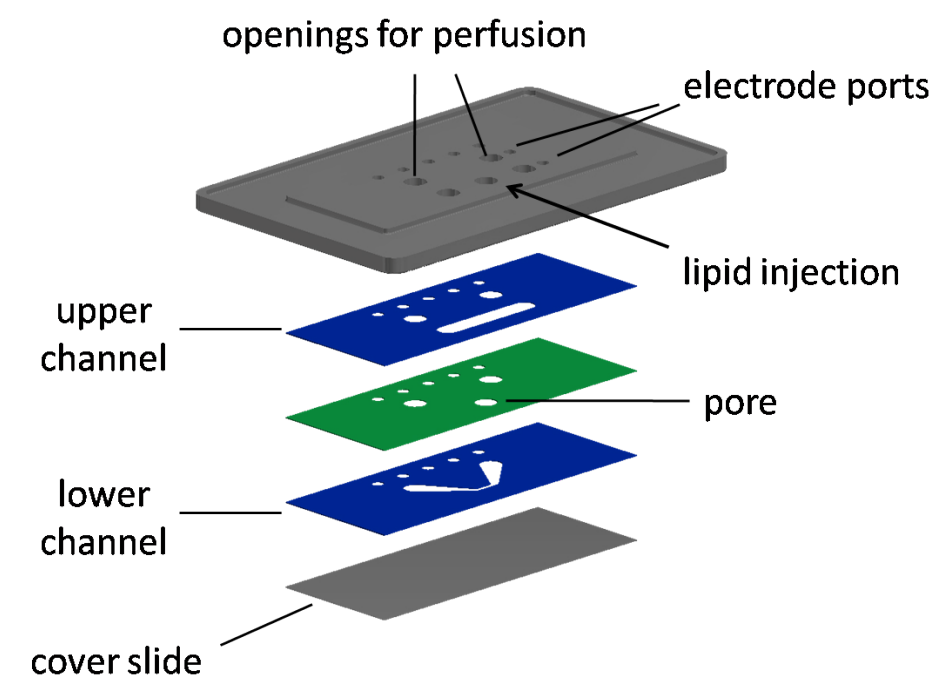

Figure 12: Bilayer Slide used for BLM preparation. The channels around the pore can be perfused individually allowing for a controlled buffer exchange in the entire chip or on either side of the membrane. The chip is conneceted to a patch clamp amplifier via electrodes to monitor bilayer formation via capacitance measurements.

HEKA, Lambrecht, Germany). This allows for monitoring BLM formation via capacitance measurements and electrophysiology measurements of membrane proteins inside the bilayer.

In order to generate BLMs, $0.2 \mu \mathrm{L}$ of the lipid mixture in dodecane were added to the Bilayer Slide which itself contained an aqueous buffer. Different buffers were used depending on the experiment. The respective buffer conditions are described in detail for every experiment. The lipids were painted over the pore using an automated pumping cycle. Bilayer formation was indicated by a capacitance larger than $50 \mathrm{pF}$.

\subsection{GUV preparation}

POPC and POPE were dissolved in chloroform and mixed in a ratio of 40 weight\% and 60 weight\%, respectively, to a final lipid concentration of $1 \mathrm{mg} / \mathrm{mL}$. $1 \mu \mathrm{L}$ $1 \mathrm{mg} / \mathrm{mL}$ Biotinyl-PE and $0.07 \mu \mathrm{L} 5 \cdot 10^{-5} \mathrm{mg} / \mathrm{mL} \mathrm{DPPE}^{\text {Atto655 }}$ in chloroform were added to give the stock solution. $60 \mu \mathrm{L}$ of this stock solution were added onto the lower electrode of a home-built electroformation chamber (figure 13) and evaporated for 30 minutes under vacuum.

$500 \mu \mathrm{L} 100 \mathrm{mM}$ sucrose solution were added to the dry lipid film and the chamber was closed with the upper electrode. Electroformation was performed for 3 hours at 


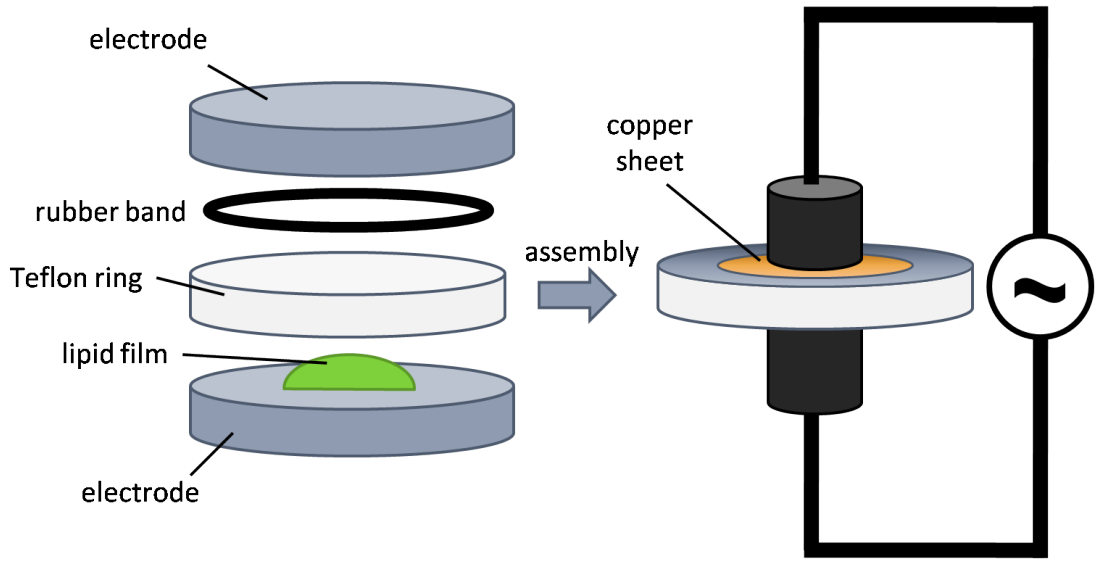

Figure 13: Home-built electroformation chamber for GUV synthesis.

$15 \mathrm{~Hz}$. Afterwards, the GUVs were collected in a $1.5 \mathrm{~mL}$ reaction tube and diluted 1:2 with $100 \mathrm{mM}$ glucose solution. Due to the density difference between sucrose and glucose solution, the GUVs sank to the bottom of the tube.

A two-sided adhesive spacer was attached to a $24 \times 50 \mathrm{~mm}$ glass cover slide. $300 \mu \mathrm{L}$ $0.02 \mathrm{mg} / \mathrm{mL}$ Streptavidin solution in phosphate buffered saline ( $(\overline{\mathrm{PBS}})(\mathrm{pH} 7.4)$ were incubated inside the spacer for one hour. The cover slide was then washed carefully with $100 \mathrm{mM}$ glucose solution. $200 \mu \mathrm{L}$ of the GUV solution from the bottom of the reaction tube were added onto the cover slide. The chamber was closed by attaching an $18 \mathrm{x} 18 \mathrm{~mm}$ cover slide on the top part of the spacer. The solution was incubated for two hours at room temperature to allow for sufficient Biotin-Streptavidin binding.

\subsection{Protein Expression, Purification and Labeling}

\subsubsection{Cytochrome B5}

TRC40/Cyctochorme-B5 complex in 4-(2-hydroxyethyl)-1-piperazineethanesulfonic acid (HEPES) buffer (50 mM HEPES KOH pH 7.4, $150 \mathrm{mM}$ potassium acetate, $10 \mathrm{mM}$ magnesium acetate, $10 \%$ glycerol, $1 \mathrm{mM}$ phenylmethylsulfonyl fluoride (PMSF), $20 \mathrm{mM}$ maltose) was provided by Fabio Villardi and Blanche Schwappach (Georg-August-University Göttingen, Germany). Cytochrome-B5 CytB5 was purified in a complex with TRC40 which gives high yield and purity and avoids degradation of the protein without further stabilization by a surfactant.

Fluorescent labeling with Alexa647 succinimidyl ester was performed at pH 8.3 in $\mathrm{NaHCO}_{3}$ buffer for 3 hours in the dark at room temperature and a 2-fold excess of fluorescent dye with regard to the amount of lysines in the protein. The la- 


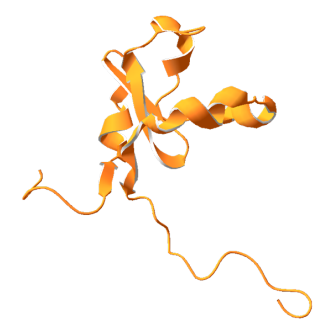

Figure 14: Cytochrome B5 monomer (depicted without transmembrane domain).

beled protein was then purified by size-exclusion chromatography using a self-packed Sephadex G-25 column (GE Healthcare, Munich, Germany).

The CytB5 crystal structure was taken from the RCSB Protein Data Bank, file 2i96.

\subsubsection{KcsA}

KcsA from Streptomyces lividans with a Cterminal hexa-histidine tag in pQE60 vector was provided by Hildgund Schrempf (University of Osnarbrück, Germany) and transformed into E.coli BL21 gold bacteria (Agilent Technologies, Waldbronn, Germany). The bacteria were grown in lysogeny broth with $0.1 \mathrm{~g} / \mathrm{L}$ ampicillin at $37^{\circ} \mathrm{C}$. At an $\mathrm{OD}_{600}=0.4$, expression was

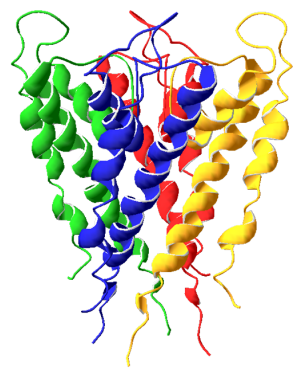

Figure 15: KcsA tetramer. induced by addition of isopropyl $\beta$-D-1-thiogalactopyranoside (IPTG) to a final concentration of $1 \mathrm{mM}$ and incubated for an additional 2 hours at $37^{\circ} \mathrm{C}$ before cells were collected by centrifugation $\left(5,000 \mathrm{~g}, 20\right.$ minutes, $\left.4{ }^{\circ} \mathrm{C}\right)$. After resuspension of the cells in lysis buffer (50 mM Tricine (pH 7.5), $150 \mathrm{mM} \mathrm{NaCl}, 5 \mathrm{mM} \mathrm{KCl}, 0.02 \mathrm{mg} / \mathrm{mL}$ DNAse, $0.2 \mathrm{mg} / \mathrm{mL}$ Lysozym, 1:100 protease inhibitor (프 $)$ mix (50 mg/mL Tame, $1.33 \mathrm{mg} / \mathrm{mL}$ Trypsin, $1 \mathrm{mg} / \mathrm{mL}$ Pepstatin A, $5 \mathrm{mg} / \mathrm{mL}$ Leupeptin, $1 \mathrm{mg} / \mathrm{mL}$ Aprotinin)), the cells were disrupted by sonification and pelleted by ultracentrifugation $\left(100,000 \mathrm{~g}, 20\right.$ minutes, $\left.4{ }^{\circ} \mathrm{C}\right)$. The pellet was resuspended in $4 \mathrm{~mL}$ lysis buffer 2 (50 mM Tricine (pH 7.5), $150 \mathrm{mM} \mathrm{NaCl}, 5 \mathrm{mM} \mathrm{KCl}$ and PI mix (1:100)). $2 \mathrm{~mL}$ solubilization buffer (50 mM Tricine (pH 7.5), $150 \mathrm{mM} \mathrm{NaCl}, 5 \mathrm{mM} \mathrm{KCl,} 20 \mathrm{mM}$ $\mathrm{DM})$ were added and the mixture was incubated on a tilting table for 2 hours at $10{ }^{\circ} \mathrm{C}$. After centrifugation at $5,000 \mathrm{~g}$ for 30 minutes at $4{ }^{\circ} \mathrm{C}$, the supernatant was loaded on a self-packed Nickel nitrioltriacetic acid (Ni-NTA column of $3 \mathrm{~mL}$ bed volume equilibrated with $30 \mathrm{~mL}$ washing buffer 1 (50 mM Tricine (pH 7.5), $150 \mathrm{mM}$ 
$\mathrm{NaCl}, 5 \mathrm{mM} \mathrm{KCl}, 5 \mathrm{mM}$ DM, $50 \mathrm{mM}$ imidazole). The protein was allowed to bind to Ni-NTA for 40 minutes. Afterwards, the column was washed with $30 \mathrm{~mL}$ of washing buffer 2 (50 mM tricine (pH 7.5), $150 \mathrm{mM} \mathrm{NaCl,} 5 \mathrm{mM} \mathrm{KCl,} 5 \mathrm{mM}$ DM, $100 \mathrm{mM}$ imidazole) and eluted with $6 \mathrm{~mL}$ elution buffer (50 mM Tricine (pH 7.5), $150 \mathrm{mM}$ $\mathrm{NaCl}, 5 \mathrm{mM} \mathrm{KCl}, 5 \mathrm{mM}$ DM, $500 \mathrm{mM}$ imidazole).

For labeling, the buffer was exchanged to PBS $(136.9 \mathrm{mM} \mathrm{NaCl}, 2.7 \mathrm{mM} \mathrm{KCl}$, $1.5 \mathrm{mM} \mathrm{KH} \mathrm{PO}_{4}, 8 \mathrm{mM} \mathrm{Na} \mathrm{HPO}_{4} \cdot 12 \mathrm{H}_{2} \mathrm{O}$, pH 7.4) with $5 \mathrm{mM}$ DM using Vivaspin columns (Satorius, Göttingen, Germany) with a membrane of $10 \mathrm{kDa}$ molecular weight cut off (MWCO). For efficient succinimidyl ester formation, the $\mathrm{pH}$ was adjusted to $\mathrm{pH} 8.3$ by adding $\mathrm{NaHCO}_{3}$ to the PBS buffer before labeling. Alexa647 succinimidyl ester was added in a 10-fold molar excess with respect to the amount of lysines present, and incubated for 3 hours in the dark at room temperature. Remaining free dye was removed via size-exclusion chromatography (Sephadex G25, GE Healthcare, Munich, Germany).

The crystal structure of KcsA was taken from the RCSB Protein Data Bank, file 1BL8.

\subsection{3 $\mathrm{EcClC}$}

EcClC from Escherichia coli (E.coli) with C-terminal hexa-histidine tag in pET28 vector was provided by Raimund Dutzler (University of Zurich, Switzerland) and transformed into E.coli BL21 gold. The protein was expressed as reported ${ }^{32}$. For purification, the pellet was resuspended in

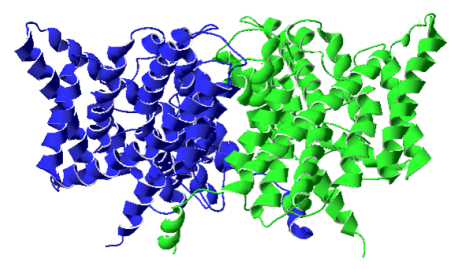

Figure 16: EcClC dimer. lysis buffer (50 mM Tris $\cdot \mathrm{HCl}(\mathrm{pH} 7.5), 150$ $\mathrm{mM} \mathrm{NaCl}, 0.02 \mathrm{mg} / \mathrm{mL}$ DNAse, $0.2 \mathrm{mg} / \mathrm{mL}$ Lysozym, 1:100 PI mix, $1 \mathrm{mM}$ PMSF, $0.1 \%$ Triton X-100) and the cells were disrupted by sonifcation. Decyl maltoside (DM) was added to the solution to a final concentration of $50 \mathrm{mM}$. The mixture was incubated for 2 hours at room temperature on a tilting table and centrifuged at $40,000 \mathrm{~g}$ for 30 minutes at $4{ }^{\circ} \mathrm{C}$. The pellet was discarded and the supernatant loaded on a self-packed Ni-NTA column of $3 \mathrm{~mL}$ bed volume that was previously equilibrated with $30 \mathrm{~mL}$ Tris buffer ( $50 \mathrm{mM}$ Tris $\mathrm{HCl}$ (pH 7.5), $150 \mathrm{mM} \mathrm{NaCl}$ ). The protein was allowed to bind to the column for 45 minutes. The column was washed with $20 \mathrm{~mL}$ washing buffer (PBS (pH 7.4), $10 \mathrm{mM}$ DM, $100 \mathrm{mM}$ imidazole) and 
2. Materials And Methods

eluted with $6 \mathrm{~mL}$ elution buffer (PBS (pH 7.4), $10 \mathrm{mM}$ DM, $400 \mathrm{mM}$ imidazole). The protein was then labeled in elution buffer with Alexa647 succinimidyl ester with a 5 -fold molar excess of dye with respect to the amount of lysines present. The $\mathrm{pH}$ of the elution buffer was adjusted to 8.3 with $1 \mathrm{M}$ aqueous $\mathrm{NaHCO}_{3}$. The remaining free dye was removed as described previously via size-exclusion chromatography.

The crystal structure of EcClC was taken from the RCSB Protein Data Bank, file 1OTS.

\subsubsection{AcrB}

AcrB from E.coli with a C-terminal histidine tag in pET24 vector was provided by Klaas Martinus Pos (Johann-WolfgangGoethe-University, Frankfurt, Germany) and transformed into E.coli BL21 gold. The bacteria were grown in lysogeny broth with $0.1 \mathrm{~g} / \mathrm{L}$ kanamycine at $37{ }^{\circ} \mathrm{C}$ to an $\mathrm{OD}_{600}$ of 0.9 . The culture was cooled to $4{ }^{\circ} \mathrm{C}$ for 20 minutes before expression, was induced by addition of IPTG to a final concentration of $0.5 \mathrm{mM}$, and incubated for an

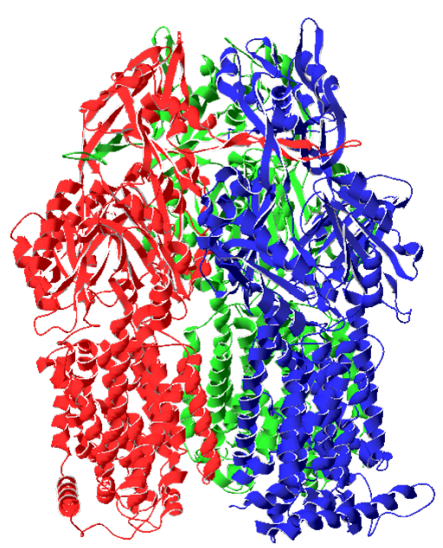

Figure 17: AcrB trimer. additional 2 hours at $37^{\circ} \mathrm{C}$. The cells were collected by centrifugation $\left(5000 \mathrm{~g}, 30\right.$ minutes, $\left.4{ }^{\circ} \mathrm{C}\right)$. All purification steps were carried out on ice. The pellet was resuspended in lysis buffer (20 mM Tris (pH 8), $500 \mathrm{mM} \mathrm{NaCl}, 2 \mathrm{mM} \mathrm{MgCl}_{2}, 0.02 \mathrm{mg} / \mathrm{mL}$ DNAse, $0.2 \mathrm{mg} / \mathrm{mL}$ Lysozym). The cells were disrupted by sonification and collected by centrifugation at $9,000 \mathrm{~g}$ for 10 minutes at $4{ }^{\circ} \mathrm{C}$. The pellet was discarded and the supernatant subjected to ultracentrifugation at $45,000 \mathrm{~g}$ for 1 hour at $4{ }^{\circ} \mathrm{C}$. The resulting pellet was resuspended in $1 \mathrm{~mL} 20 \mathrm{mM}$ Tris (pH 8) and $500 \mathrm{mM} \mathrm{NaCl} .1 \mathrm{~mL} 2 \mathrm{~mol} / \mathrm{L} \mathrm{DM}$ solution and $2.2 \mathrm{~mL}$ buffer A (10 mM sodium piperazine-N,N'-bis(2-ethanesulfonic acid) (PIPES) (pH 8), $190 \mathrm{mM} \mathrm{NaCl}, 10 \mathrm{mM} \mathrm{KCl,} 10 \mathrm{mM}$ imidazole, 10 \% Glycerol, $1 \mathrm{mM} \mathrm{DM}$ ) were added and the mixture was rotated slowly for $2 \mathrm{~h}$ on ice in the cold room $\left(10{ }^{\circ} \mathrm{C}\right)$. Afterwards, the sample was spun at $45,000 \mathrm{~g}$ for 1 hour at $4{ }^{\circ} \mathrm{C}$. The pellet was discarded and the supernatant was loaded on a self-packed Ni-NTA column of $3 \mathrm{~mL}$ bed volume equilibrated with $10 \mathrm{~mL}$ buffer $\mathrm{A}$.

AcrB labeling was performed during Ni-NTA affinity chromatography. Therefore, 
$75 \mu \mathrm{g}$ Alexa647 maleimide (50-fold molar excess with respect to the number of cysteines present) were added to the solution on the Ni-NTA resin. The $\mathrm{pH}$ was adjusted to 7.2 by addition of $4 \mathrm{mM} \mathrm{HCl}$, to allow for efficient labeling of the cysteine groups. The mixture was incubated in the dark on a tilting table overnight on ice in the cold room $\left(10{ }^{\circ} \mathrm{C}\right)$. Excess free dye was removed by washing the column with $60 \mathrm{~mL}$ washing buffer (10 mM sodium PIPES, pH 8, $190 \mathrm{mM} \mathrm{NaCl}, 10 \mathrm{mM}$ $\mathrm{KCl}, 10 \%$ glycerol, $1 \mathrm{mM} \mathrm{DM}, \mathrm{pH} 8)$. Afterwards, non-specifically bound proteins were removed with $40 \mathrm{~mL}$ washing buffer A (same composition as washing buffer + $10 \mathrm{mM}$ imidazole, pH 8) and $30 \mathrm{~mL}$ washing buffer B (200 mM NaCl, $10 \%$ glycerol, $1 \mathrm{mM} \mathrm{DM}, 50+\mathrm{mM}$ imidazole, $\mathrm{pH}+7)$. The protein was eluted with $6 \mathrm{~mL}$ elution buffer (200 mM NaCl, $10 \%$ glycerol, 1 mM DM, 200 mM imidazole, pH 5).

The crystal structure of AcrB was taken from the RCSB Protein Data Bank, file 2GIF.

\subsection{LUV Preparation and SNARE-mediated Vesicle Fusion}

Large Unilamellar Vesicles (LUVS) were prepared by extrusion. Therefore $25 \mu \mathrm{L}$ of $25 \mathrm{mg} / \mathrm{mL}$ POPE and $16 \mu \mathrm{L}$ of $25 \mathrm{mg} / \mathrm{mL}$ POPC solution in chloroform were mixed and the solvent was evaporated. The lipids were then resuspended in $800 \mu \mathrm{L}$ PBS (pH 7.4) under vigorous shaking for 60 minutes, which generates Multilamellar Vesicles (MLVs). Afterwards, the mixture was extruded for 350 cycles using a lipid extruder from Avanti Polar lipids (Alabaster, AL, USA) with a $100 \mathrm{~nm}$ polycarbonate membrane (GE Healthcare / Whatman, Piscataway, NJ, USA). By forcing the MLVs through the polycarbonate membrane, the outer layers of the onion-like MLVs are "peeled off" resulting in the formation of large unilamellar vesilces of $100 \mathrm{~nm}$ diameter.

In order to incorporate fluorescently labeled KcsA into LUVs, the protein was subjected to a buffer exchange from Tricine (50 mM Tricine, $150 \mathrm{mM} \mathrm{NaCl}, 5 \mathrm{mM}$ $\mathrm{KCl}, 500 \mathrm{mM}$ Imidazole, $5 \mathrm{mM}$ DM, pH 7.5) to PBS (pH 7.4 with an additional $150 \mathrm{mM} \mathrm{NaCl}$ and $50 \mathrm{mM}$ octyl glycoside) before labeling using Vivaspin columns with $10 \mathrm{kDa}$ molecular weight cut-off (MWCO) membrane. Labeling of the protein was done as described as before. $1 \mathrm{~mL}$ of a $10 \mu \mathrm{M}$ fluorescently labeled protein solution were then mixed with the LUVs. $20 \mu \mathrm{L}$ of a $156 \mu \mathrm{M}$ unlabeled Synaptobrevin-2 Syb solution provided by Geert van den Bogaart and Reinhard Jahn (Max-Planck-Institute for Biophysical Chemistry, Göttingen, Germany) were added. The mixture was dialyzed in the dark overnight in a dialysis tube with 
$3 \mathrm{kDa}$ pore size at $10{ }^{\circ} \mathrm{C}$ against PBS containing Biobeads SM-2 (Biorad, Munich, Germany) to remove the detergent. Afterwards, the LUV solution was purified via size-exclusion chromatography to remove remaining detergent and free dye.

Reconstitution of fluorescently labeled EcClC into POPC/POPE LUVs was done as described for KcsA. AcrB was reconstituted following the protocol from ref. ${ }^{33}$. Additionally, Syb was added to the membrane protein-LUV mixture and the vesicles were purified via size-exclusion chromatography after dialysis as before for KcsA reconstitution.

Fluorescently labeled CytB5 was reconstituted into LUVs by mixing $200 \mu \mathrm{L}$ of $0.3 \mu \mathrm{M}$ CytB5 solution with $10 \mu \mathrm{L}$ of $156 \mu \mathrm{M}$ Syb and $400 \mu \mathrm{L}$ LUV solution. The mixture was incubated in the dark overnight at $4{ }^{\circ} \mathrm{C}$.

$\Delta \mathrm{N}$ complex ${ }^{34}$ provided by Geert van den Bogaart and Reinhard Jahn was added to the chip to a final concentration of $0.44 \mu \mathrm{M}$. The used $\Delta \mathrm{N}$ complex consists of an equimolar ratio of SNAP25, Syntaxin-1A residues 183-288 and Synaptobrevin-2 residues 49-96. The Synaptobrevin-2 fragment stabilizes the acceptor complex of SNAP-25 and Syntaxin-1A. The $\Delta \mathrm{N}$ complex was allowed to incorporate into the bilayer for 15 minutes before adding $20 \mu \mathrm{L}$ of the vesicle solution. The mixture was then equilibrated for another 10 minutes to allow for SNARE fusion. This time was found to be sufficient to yield single-molecule concentration of labeled proteins in the BLM.

\subsection{Electrophysiology}

Electrophysiology allows for measuring the electrical properties of proteins in artificial membranes or of whole biological cells. It measures the voltage change or electric current induced by opening and closing of protein channels, which allows for ion conduction through their internal pore.

For electrophysiology measurements, KcsA was purified, labeled and reconstituted into vesicles with Syb as described above. A BLM with $4 \mathrm{mg} / \mathrm{mL}$ POPC, $6 \mathrm{mg} / \mathrm{mL}$ POPE and $3 \mathrm{mg} / \mathrm{mL}$ DOPG was formed as described using the Ionovation Bilayer Explorer in PBS buffer with $400 \mathrm{mM} \mathrm{KCl} \mathrm{(pH} \mathrm{4).} \mathrm{The} \mathrm{lipid} \mathrm{mixture} \mathrm{and} \mathrm{buffer} \mathrm{were}$ chosen on the basis of refs. $\frac{[35}{36}$ and ${ }^{37]}$. $\Delta \mathrm{N}$ complex was added and incubated with the bilayer for 15 minutes. Vesicles were added and fused with the bilayer as described previously. For electrophysiology measurements, a patch clamp amplifier (EPC 10, HEKA, Lambrecht, Germany) was used. Membrane voltages were clamped to $+200 \mathrm{mV}$ or $-200 \mathrm{mV}$. Currents were filtered at $2 \mathrm{kHz}$ and sampled at $10 \mathrm{kHz}$. 
For display, traces were low-pass filtered with a digital Bessel filter $(250$ to $200 \mathrm{~Hz}$ cut-off) with a notch at $50 \mathrm{~Hz}$ to remove line-noise. Afterwards, $1 \mathrm{mM}$ tetra butyl ammonium (TBA) was added to block the KcsA channels. In control experiments, a POPC/POPE BLM in the same buffer without protein was measured. Additionally, KcsA was added directly to the chip and electrophysiology was performed. BLM, directly added KcsA, and blocked KcsA reconstituted via SNARE-mediated vesicle fusion were sampled as described before using $+100 \mathrm{mV}$ and $-100 \mathrm{mV}$ pulses.

\subsection{Dual-Focus Fluorescence Correlation Spectroscopy}

\subsubsection{Fluorescence}

Many spectroscopic techniques are based on the principle of luminescence. Luminescence describes the time-delayed emission of light after absorption of energy. In principle, two processes can be distinguished. These processes, fluorescence and phosphorescence, differ in their respective excited states (singlet vs. triplet) which is also reflected in the time scales on which they occur. The respective excitation and emission processes can be visualized with a Jablonski diagram (figure 18).

Upon irradiation with photons of energy $h \nu_{A}$, a molecule absorbs this energy and is thereby excited from the ground (singlet) state $S_{0}$ into an electronic level of higher energy, i.e. the singlet states $S_{1}$ or higher. At each of these electronic levels, the molecule can be excited into different vibrational states $(0,1,2$, etc. in figure 18).

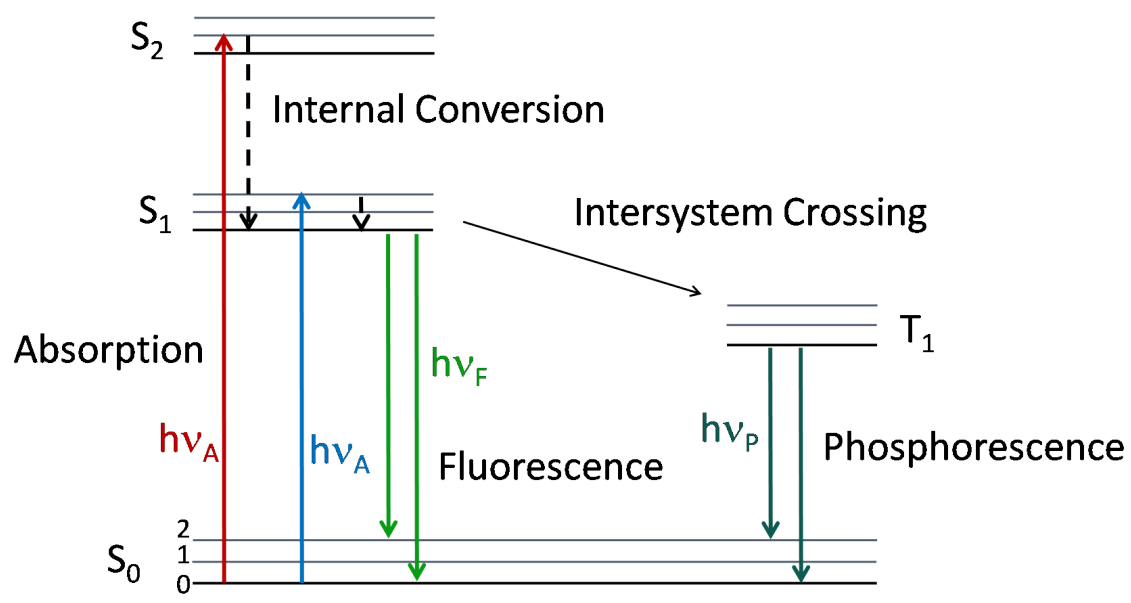

Figure 18: Jablonski diagram (adapted from ref. ${ }^{[38]}$ ).

After excitation, there are different options for emitting the absorbed energy. First of all, radiationless transitions can occur from an excited electronic and vibrational 
state into the lowest vibrational state of $S_{1}$. These radiationless transitions are called internal conversion. Secondly, the molecule can relax from the lowest vibrational state of $S_{1}$ into the ground state $S_{0}$ under emission of a photon of energy $h \nu_{F}$. During this process, the electron's spin remains unchanged resulting in a rapid transition. Fluorescence lifetimes are usually on the order of $10^{-9}-10^{-8} \mathrm{~s}$ while internal conversion from a higher vibrational state into the vibrational ground state of $S_{1}$ typically occurs within $10^{-12}$ seconds or less. Thus, internal conversion is usually complete before emission.

The third option after reaching the vibrational ground state of $S_{1}$ is, that the fluorophore can transfer into the first triplet state $T_{1}$. This process is inherently connected with a spin inversion of the electron and is called intersystem crossing. Since spin change is mostly prohibited, the probability for this process to occur is lower than the relaxation options that do not require reversal of the spin. The emission from the triplet state $T_{1}$ to the ground state $S_{0}$ is called phosphorescence and occurs on longer time scales $\left(10^{-6} \mathrm{~s}\right)$ compared to fluorescence since the electron spin has to be reversed again in order to return to $S_{0}$.

The energy of the emitted light is generally lower than the energy of the excitation. Thus, fluorescence and phosphorescence occur at longer wavelengths with respect to the excitation light. This shift to longer wavelengths, i.e. lower energies, is called Stokes shift.

The fluorophore can be characterized in terms of its quantum yield and fluorescence lifetime. The quantum yield $Q$ is defined as the amount of emitted photons $N_{e m}$ relative to the amount of absorbed photons $N_{a b s}$ :

$$
Q=\frac{N_{e m}}{N_{a b s}}
$$

The fluorescence lifetime $\tau$ is the average time, for which a molecule remains in an excited state before relaxation into the ground state. Typically, the time course of this relaxation follows an exponential law,

$$
I(t)=I_{0} \exp \left(-\frac{t}{\tau}\right)
$$

where $I_{0}$ and $I(t)$ denote the intensities immediately after excitation and at time $t$, respectively. Typical fluorescence lifetimes are on the order of nanoseconds. 


\subsubsection{Setup}

The principal setup used for diffusion measurements is described in figure 19. It is a commercially available confocal microscopy system (MicroTime 200 with dual-focus option, PicoQuant GmbH, Berlin, Germany). For excitation, the light of two identical, linearly polarized pulsed diode lasers (wavelength $640 \mathrm{~nm}$, pulse duration $50 \mathrm{ps}$ Full Width Half Maximum ( $(\overline{F W H M})$ ) is combined by a polarizing beam splitter. Both lasers are pulsed alternately with a repetition rate of $40 \mathrm{MHz}$ (pulsed interleaved excitation $(\underline{\mathrm{PIE}})^{39}$ ). The continuous wave laser power was adjusted to $3 \mu \mathrm{W}$ each.

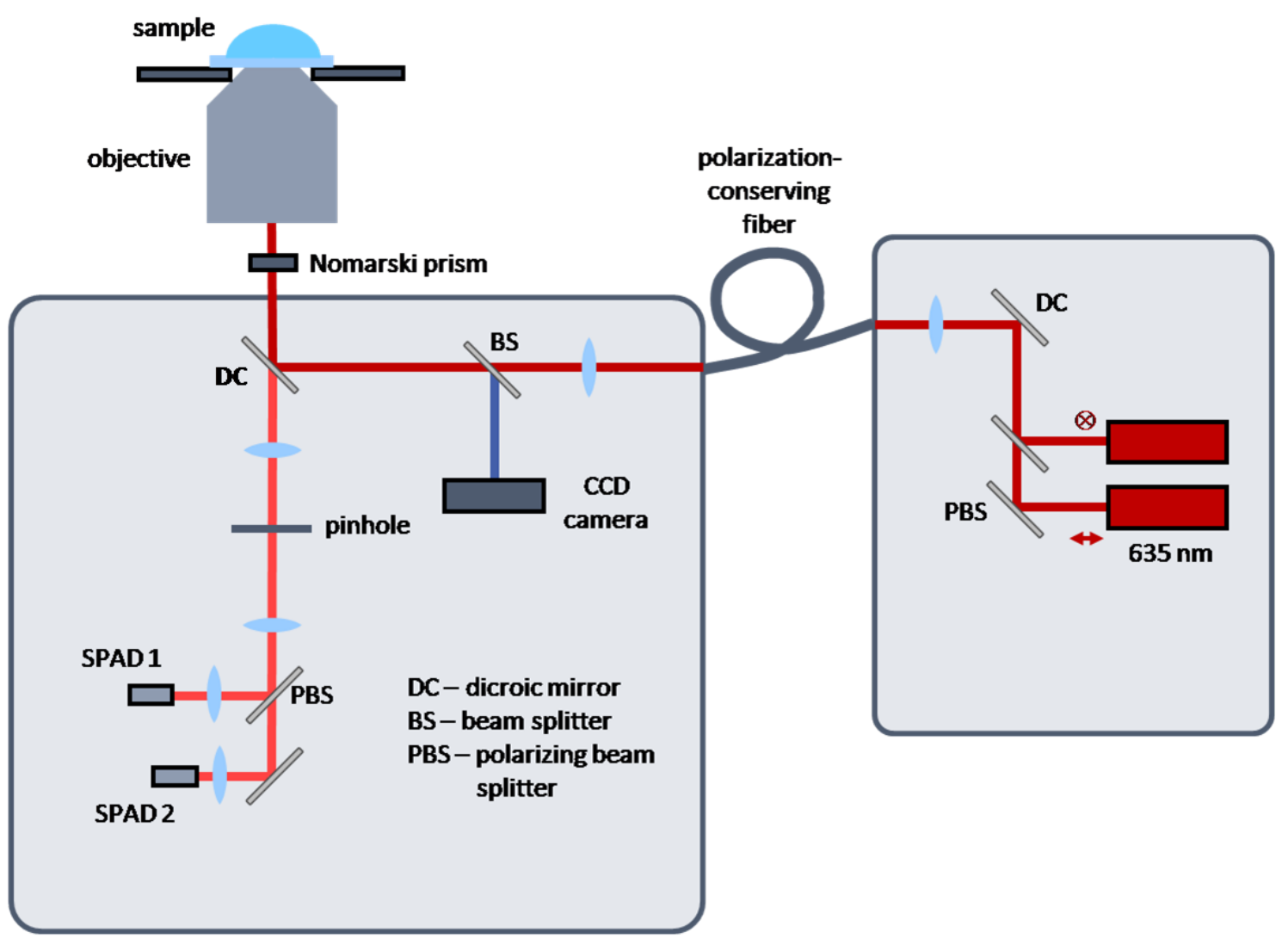

Figure 19: Dual-Focus FCS Setup.

Both beams are coupled into a polarization-maintaining single mode fiber. At the fiber output, the light is collimated and reflected by a dichroic mirror (FITC/TRITC Chroma Technology, Rockingham, VT, USA) towards the microscope's objective (UPLSAPO 60× W, 1.2 N.A., Olympus Deutschland GmbH, Hamburg, Germany). Before entering the objective, the light passes through a Nomarski prism which deflects the beams into slighly different directions, according to their polarization. After focusing the light through the objective, two overlapping but laterally shiftet 
foci of $450 \mathrm{~nm}$ distance are generated. The exact interfocal distance was determined by performing $2 \mathrm{fFCS}$ on a solution of fluorescent polymer beads with known radius (for details, see ref. ${ }^{40}$ ). Although three-dimensional diffusion processes are used for calibration, the distance obtained from the respective fits is still valid for membrane measurements in two dimensions since it corresponds to the actual focus distance, which is independent of the dimensionality 41.

Fluorescence is collected by the same objective, passed through a dichroic mirror, and focused onto a pinhole of $150 \mu \mathrm{m}$ diameter. Afterwards, the light is collimated, split by a 50/50 beam splitter, and focused onto two Single Photon Avalanche Diodes (SPADS) (two SPCM-AQR-13, PerkinElmer Optoelectronics, Wiesbaden, Germany). The detected photons of both APDs are recorded independently by single-photon counting electronics (HydraHarp 400, PicoQuant GmbH, Berlin, Germany) with an absolute temporal resolution of two picoseconds on a common time frame (time-correlated single photon counting (TCSPC) $)$. The principal of TCSPC is explained in more detail in the following section.

Using PIE and TCSPC, each fluorescene photon can be associated with the laser pulse that excited it, i.e. in which focus it was excited. Thus, autocorrelation functions for each focus and a crosscorrelation function between the foci can be calculated with a dedicated software algorithm ${ }^{42}$. Only photon pairs which have been detected in both avalanche photodiodes (APDs) are correlated to avoid the influence of afterpulsing effects. Overall, four correlation curves (two auto- and two crosscorrelation curves) are calculated taking into account the temporal order of the correlated photon pairs (i.e. whether the first photon was excited by the first laser and the second by the second laser pulse or vice versa). Generating the auto- and crosscorrelation curves is illustrated in figure 20.

The photons generated by laser pulse 1 and detected in detector 1 (P1D1) are correlated with the photons generated by pulse 1 and detected in detector 2 (P1D2) and vice versa. The two resulting $\mathrm{ACF}$ are summed up to yield ACF 1 . ACF 2 is generated in a similar manner by only considering photons generated by the second laser pulse, i.e. addition of the correlation functions for P2D1 + P2D2 and P2D2 + P2D1. Accordingly, four Crosscorrelation Functions (CCFs) can be calculated by correlating photons generated by different laser pulses (P1 and P2) as shown in figure 20. The resulting CCFs can then be summarized as well. The addition is done to generate two CCFs during evaluation, and again to yield one CCF for display. Finally, fitting of the data is done with the model curves presented in the next section. 


$\left.\begin{array}{|c|c|}\text { Correlation function } & \text { Pulse (P) and Detector (D) } \\ \hline \text { ACF 1 } & \begin{array}{l}\text { P1D1 + P1D2 } \\ \text { P1D2 + P1D1 }\end{array}\end{array}\right\}$ addition

Figure 20: Generation of auto- and crosscorrelation functions. Listed are the respective laser pulses $(P)$ and detectors $(D)$. For the two- and three-dimensional diffusion processes studied in this thesis, averaging the different correlations is possible as indicated.

\subsubsection{Time-Correlated Single Photon Counting (TCSPC)}

Time-correlated single photon counting (TCSPC) ${ }^{43[44}$ is a statistical method commonly used for determining fluorescence lifetimes. It allows for correlating the photon arrival times with the respective laser pulses with high accuracy. In 2fFCS, it is used to link the detected photons to the exciting laser pulse and thus determine in which focus they were generated.

For TCSPC, the sample is excited in such a way, that the probability of detecting a photon per laser pulse is much less than one. The time between excitation pulse and photon detection is measured and stored in a histogram (figure 21).

The photon detection rate needs to be $1 \%$ or smaller, since a more frequent arrival of photons influences the statistics which leads to a distortion of the resulting signal ("pile-up" effect). Shorter photon arrival times will be much more prominent than longer ones. Consequently, low countrates are mandatory to also adequately register photons with longer arrival times.

The time delay between excitation and emission can be measured using special electronics (figure 22). The emitted photon detected on the SPAD triggers a Constant Fraction Discriminator (CFD). Additionally, a second CFD is used to measure a timing reference pulse of the light source. The output pulses of the CFDs are then used as start and stop pulses of a time-to-amplitude converter (TAC). The TAC consists of a current source which charges a capacitor. The start and stop pulses switch the current on and off, respectively. Assuming a constant current in the in- 


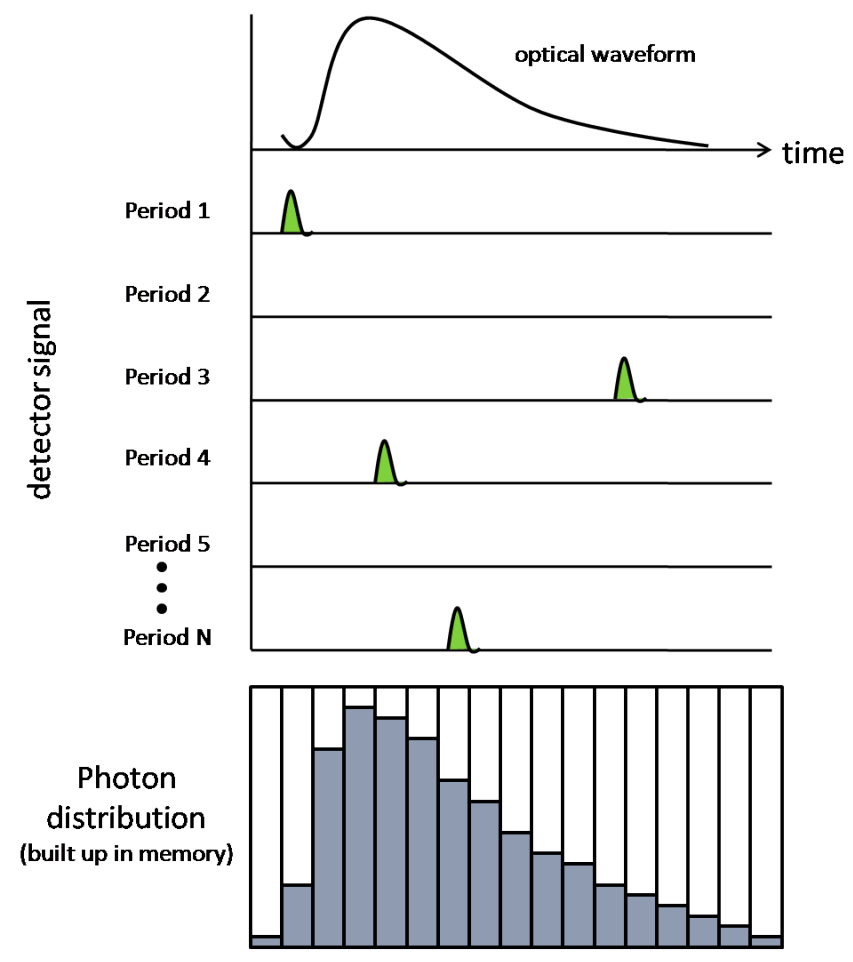

Figure 21: Principle of time-correlated single photon counting (TCSPC). Image adapted from ref. 44 .

terval between start and stop, the final voltage on the capacitor is proportional to the time between excitation (start) and emission (stop). The TAC output voltage is passed through an amplifier (AMP), which has a variable gain and offset and selects a small time window within the conversion range of the TAC. The amplified signal is then sent through an analog-to-digital converter (ADC), which converts the voltage to a numerical value and thereby resolves the TAC signal into a large number of time channels which all have the same width. This data is then stored in a histogram.

Most TCSPC systems work in reversed mode, i.e. a detected photon is used as a start signal for the TAC while the excitation pulse stops the TAC. This is advantageous for two reasons. First, the TAC needs to be reset to zero before each new pulse, which takes a certain time. Thus, the TAC is constantly resetting if the signals arrive too rapidly and accurate photon counting is impossible. Since emission events happen much less frequently compared to excitation events, TAC resetting is unproblematic with the reserved start-stop configuration. Second, dead-times of the detectors after photon arrival do not distort the resulting signal in the reversed mode, since detection events are sufficiently rare.

For newer systems, operation in reversed mode is not necessary anymore, since the 


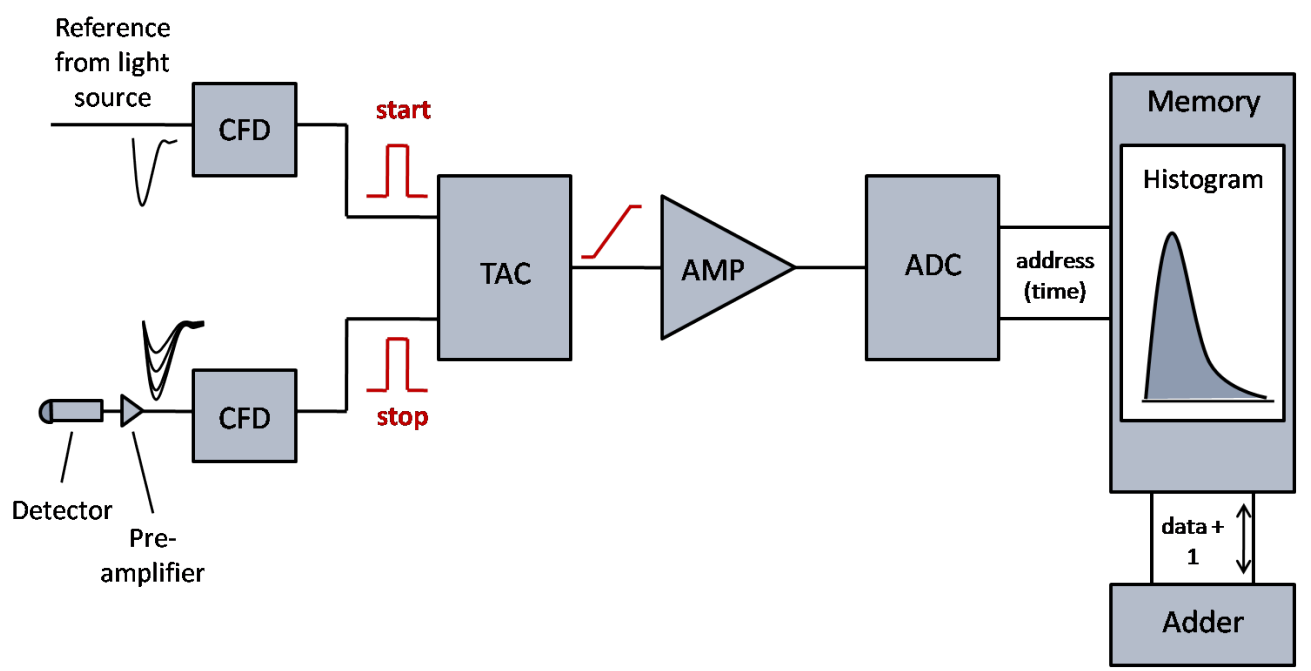

Figure 22: Classical TCSPC setup. In reversed mode, start and stop pulses are inverted, i.e. the TAC is started by the emission pulse instead of the excitation. Image adapted from ref. [44].

electronics are sufficiently fast and the detector dead-times are sufficiently small to allow for highly accurate photon counting even using the classical TCSPC setup (figure 22).

\subsubsection{Theoretical Background}

\subsubsection{2fFCS in Solution}

In 2fFCS, photons originating from two identical but laterally shifted foci are detected. Since it is possible to determine which photon was generated in which focus by using PIE and TCSPC, ACFs for each detection volume can be calculated. The autocorrelation function is an expression for the probability to detect a photon from the same molecule at a time $t$ and at a later time $t+\tau$. Exemplary autocorrelation curves are depicted in figure 23. The shape of the correlation curves visualizes the probability distribution: at short lag times, a high correlation is observed because the probability to detect a photon from the same molecule at two times $t$ and $t+\tau \approx t$ is high. As $\tau$ increases $(t+\tau>>t)$, the probability to detect a photon from the same molecule decreases, resulting in a characteristic decay of the correlation curve. Since the decrease in probability depends on the molecule's diffusion coefficient, $D$ can be extracted from the decay of the correlation curves. 


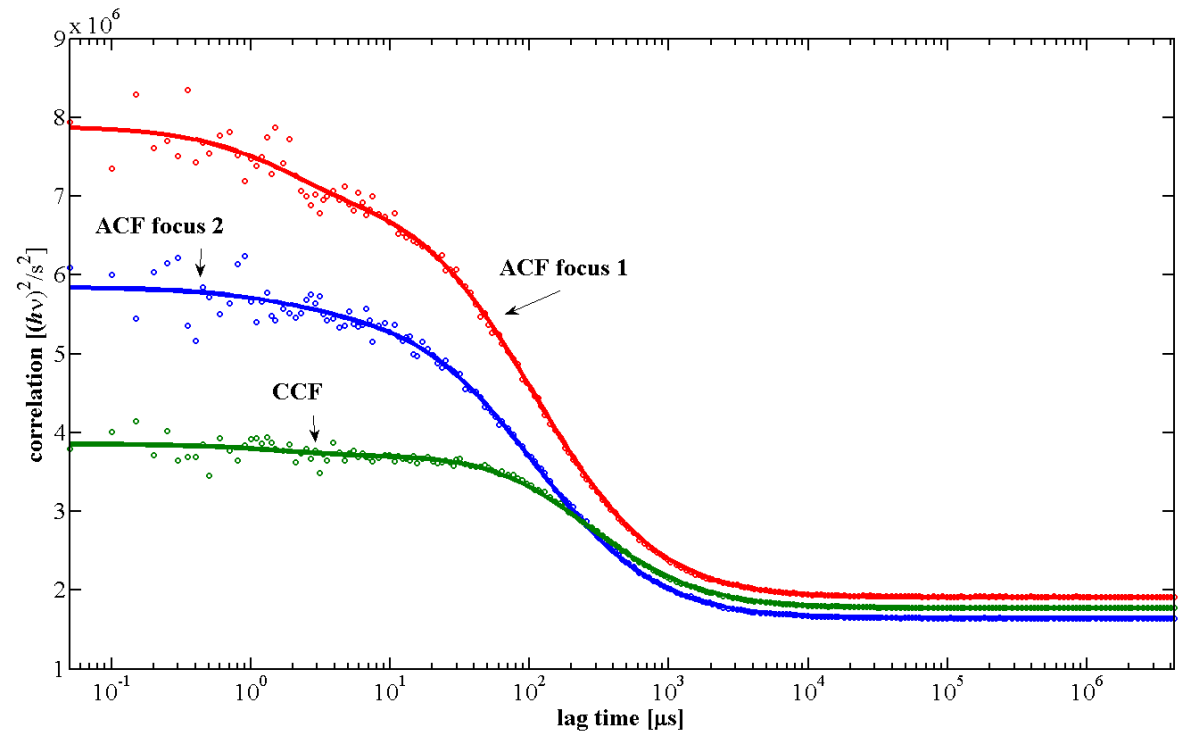

Figure 23: Exemplary auto- and crosscorrelation functions for OregonGreen 488 in solution.

The autocorrelation function is given by

$$
g(\tau)=\langle I(t) I(t+\tau)\rangle
$$

where $I(t)$ and $I(t+\tau)$ denote the fluorescence intensities at times $t$ and $t+\tau$, respectively, and the angular brackets denote averaging over time $t$. The autocorrelation function can also be expressed in terms of the diffusion of molecules into and out of the focus. Therefore, we consider the probability to detect a photon at a position $\vec{r}_{1}$ in one detection volume, which is given by the Molecule Detection Function (MDF) $U\left(\vec{r}_{1}\right)$ at this position. The probability to detect a photon at a different position $\vec{r}_{2}$ is described by the MDF at the respective new position $U\left(\vec{r}_{2}\right)$. The probability, that a molecule diffuses from position $\vec{r}_{1}$ to position $\vec{r}_{2}$ within a time $\tau$ can be described by the fundamental solution of the diffusion equation

$$
G(\vec{r}, \tau)=\frac{1}{(4 \pi D \tau)^{\frac{3}{2}}} \exp \left(-\frac{\left|\vec{r}_{2}-\vec{r}_{1}\right|^{2}}{4 D \tau}\right)
$$

where $D$ denotes the molecule's diffusion coefficient. Therefore, the autocorrelation $g(t)$ can be expressed as the product of these probability contributions. Averaging over all possible initial and final positions of the molecule yields

$$
g(t)=c \epsilon^{2} \int_{V} d \vec{r}_{1} \int_{V} d \vec{r}_{2} U\left(\vec{r}_{2}\right) \frac{1}{(4 \pi D t)^{\frac{3}{2}}} \exp \left(-\frac{\left|\vec{r}_{2}-\vec{r}_{1}\right|^{2}}{4 D t}\right) U\left(\vec{r}_{1}\right)
$$


where $V$ is the sample volume, $c$ is the concentration and $\epsilon$ denotes the overall detection efficiencies in the corresponding detection volumes (i.e. the two foci). While in the ACF, photons from one and the same detection volume are correlated, the $\mathrm{CCF}$ correlates photons from one detection volume with those detected in the other one and takes into account the spatial separation between the two foci. For a lateral shift $\delta$ along the $x$-axis perpendicular to the optical axis of the microscope, the CCF is given by

$$
g(t, \delta)=c \epsilon^{2} \int d \vec{r}_{1} \int d \vec{r}_{2} U\left(\vec{r}_{2}\right) \frac{1}{(4 \pi D t)^{\frac{3}{2}}} \exp \left(-\frac{\left(\vec{r}_{1}-\vec{r}_{2}-\hat{x} \delta\right)^{2}}{4 D t}\right) U\left(\vec{r}_{1}\right)
$$

where $\hat{x}$ denotes the unit vector along the $x$-axis. $\epsilon^{2}$ is in this case the square of the geometric mean of the total detection efficiency over both detection volumes.

In order to accurately evaluate 2fFCS data, it is crucial to use an appropriate model function for the MDF. It was shown that a Gauss-Lorentzian function is a suitable approximation for the MDF of a confocal microscope ${ }^{30}$ :

$$
U(\vec{r})=\frac{\kappa(z)}{w^{2}(z)} \exp \left(-\frac{2}{w^{2}(z)}\left[x^{2}+y^{2}\right]\right)
$$

Here, $x$ and $y$ denote transversal coordinates perpendicular to the optical axis $z=0$. In essence, equation 10 is a modified three-dimensional Gaussian function and states that, in each plane perpendicular to the optical axis, the MDF can be approximated by a Gaussian distribution of width $w(z)$ and amplitude $\frac{\kappa(z)}{w^{2}(z)}$. The width is given by

$$
w(z)=w_{0}\left[1+\left(\frac{\lambda_{e x} z}{\pi w_{0}^{2} n}\right)^{2}\right]^{\frac{1}{2}}
$$

which is the scalar approximation for the radius of a diverging laser beam with beam waist radius $w_{0}$. The function $\kappa(z)$ is defined as

$$
k(z)=1-\exp \left(-\frac{2 \alpha^{2}}{R^{2}(z)}\right)
$$

where $R(z)$ is given by

$$
R(z)=R_{0}\left[1+\left(\frac{\lambda_{e m} z}{\pi R_{0}^{2} n}\right)^{2}\right]^{\frac{1}{2}}
$$

$\lambda_{e x}$ and $\lambda_{e m}$ denote the excitation and center emission wavelength, respectively. $n$ is the refractive index of the immersion medium (water), $\alpha$ is the radius of the confocal aperture divided by the magnification, and $w_{0}$ and $R_{0}$ are model parameters. 
The MDF (equation 10) and the parameterization (equation 11-equation 13) can be substituted into equation 9 which yields the following expression for the CCF:

$$
g(t, \delta)=\frac{c \epsilon^{2}}{4} \sqrt{\frac{\pi}{D t}} \int d z_{1} \int d z_{2} \frac{\kappa\left(z_{1}\right) \kappa\left(z_{2}\right) \exp \left[-\frac{\left(z_{2}-z_{1}\right)^{2}}{4 D t}-\frac{2 \delta^{2}}{8 D t+w^{2}\left(z_{1}\right)+w^{2}\left(z_{2}\right)}\right]}{8 D t+w^{2}\left(z_{1}\right)+w^{2}\left(z_{2}\right)}
$$

The resulting model curve is globally fitted with a linear least-square model with $c \epsilon^{2}, D, w_{0}$ and $R$ as fit parameters. The distance $\delta$ between the foci is defined by the properies of the Nomarski prism and can be determined precisely by 2fFCS measurements of dyes or polymer beads.

\subsubsection{2fFCS in Lipid Bilayers}

As opposed to diffusion in solution, where molecules can move randomly in three dimensions, diffusion in lipid membranes is limited to the bilayer plane and is therefore a two-dimensional process. As in the three-dimensional case, the $\operatorname{MDF} U(\vec{r})$ describes the position-dependent probability to excite and detect a photon at a given position. If $U_{j}(\vec{r})$ denotes the MDF of the $j$ th focus, the lag-time dependent part of the auto- and crosscorrelation functions for the diffusion of molecues within a plane placed at a position $z$ along the optical axis is given by

$$
g_{j k}(t, z)=\frac{1}{4 D t} \int d \vec{\rho}_{2} \int d \vec{\rho}_{1} U_{k}\left(\vec{\rho}_{2}, z\right) \exp \left(-\frac{\left|\vec{\rho}_{1}-\vec{\rho}_{2}-\vec{\delta}_{j k}\right|^{2}}{4 D t}\right) U_{j}\left(\vec{\rho}_{1}, z\right)
$$

where $g_{j k}(t, z)$ denotes the correlation function of the fluorescence signal from focus $j$ against that from focus $k, D$ is the diffusion coeffcient and $\vec{\rho}_{1,2}$ are radial position vectors perpendicular to the optical axis. $\vec{\delta}_{j k}$ is the lateral distance between the foci. If the two foci overlap exactly $(j=k)$, the focus distance is $\vec{\delta}_{j k}=0$. For laterally shifted foci $(j \neq k)$, the focus distance vector becomes non-zero $\vec{\delta}_{j k}=\delta$. Both integrations extend over the whole plane.

For three-dimensional diffusion in solution, the approximation that the molecular detection function $U(\vec{r})$ is the same for both detection volumes holds, despite focus distortion or slight axial shifts of the foci. In case of two-dimensional membrane diffusion, however, this assumption is not valid anymore. Because the foci have different diameters, individual MDFs $U_{j}$ and $U_{k}$ need to be considered for each focus ( $j$ and $k$ ), respectively. 
In the next step, the calculated correlation functions are fitted with a 2fFCS diffusion model. For diffusion within a plane, the radial distribution of the MDFs at position $z$ can be approximated by a two-dimensional Gaussian distribution function

$$
U_{j}(\vec{\rho}, z) \propto \exp \left(-\frac{2 \vec{\rho}^{2}}{w_{j}^{2}}\right)
$$

where $w_{j}$ denotes the width of the Gaussian distribution for the $j$ th focus. Using this assumption, the correlation functions can (up to a constant factor) be expressed as

$$
\tilde{g}_{j k}(t, z)=\frac{1}{w_{j}^{2}+w_{k}^{2}+8 D t} \exp \left(-\frac{2 \delta_{j k}^{2}}{w_{j}^{2}+w_{k}^{2}+8 D t}\right)
$$

with $j$ and $k$ both being either to 1 or 2 . The model functions $\tilde{g}_{j k}(t, z)$ are used for fitting the experimental data, where $\tilde{g}_{11}(t, z)$ and $\tilde{g}_{22}(t, z)$ are the autocorrelation functions, and $\tilde{g}_{12}(t, z)$ and $\tilde{g}_{21}(t, z)$ are the crosscorrelation functions. When globally fitting the correlation curves, the beam waist diameters $w_{1}$ and $w_{2}$ and the diffusion coefficient $D$ are used as fit parameters. 


\subsubsection{Performance of 2fFCS in Lipid Membrane Measure- ments}

To study the performance of 2fFCS for diffusion measurements in bilayers and estimate the impact of mispositioning the foci with respect to the bilayer plane, model calculations of diffusion measurements at different $z$ positions were performed. The parameters used in the calculations were set to match the experimental ones. The numerical aperture of the objective is 1.2 and the objective is considered optically perfect for a refractive index of 1.33, i.e. for focusing and imaging in water. The focal distance of the objective is assumed to be $3 \mathrm{~mm}$ and the focal distance of the tube lens is $180 \mathrm{~mm}$, which corresponds to a magnification of $60 \times$ at the confocal aperture. The aperture diameter is set to $150 \mu \mathrm{m}$. The excitation wavelength is $640 \mathrm{~nm}$, the peak emission wavelength is $670 \mathrm{~nm}$. Moreover, it is assumed that the laser beam focused through the objective into the sample has a Gaussian intensity profile. In order to check how this focusing affects the 2fFCS measurement, the $1 / e^{2}$-radius of the Gaussian profile was varied from $1.25 \mathrm{~mm}$ to $3.5 \mathrm{~mm}$, thus covering a range from relaxed to nearly diffraction-limited focusing which is illustrated in figure 24 .

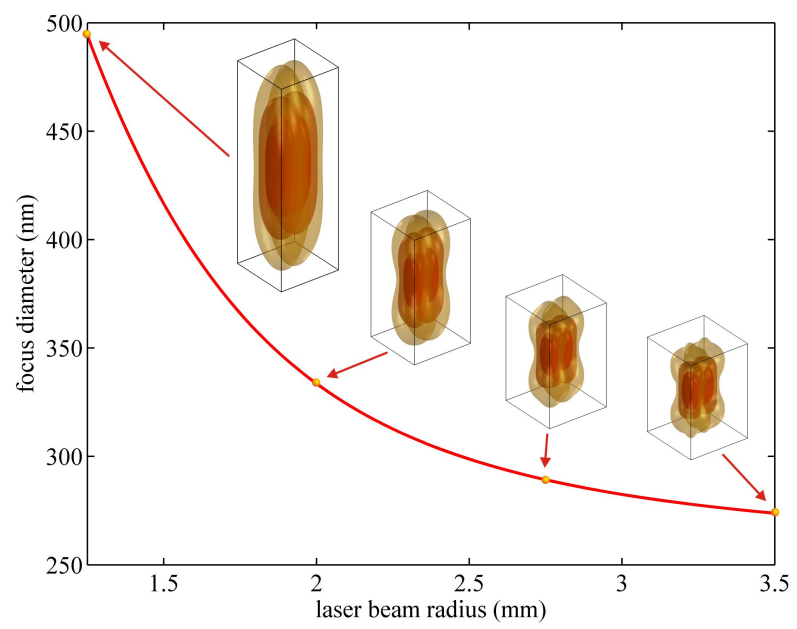

Figure 24: Connection between the radius of the laser beam which is coupled into the objective and the diameter of the resulting focus in sample space. Insets show the shape of the two overlapping MDFs for selected laser beam radii. Boxes have the same transversal size of $1.2 \mu \mathrm{m}$ by $1.6 \mu \mathrm{m}$. Shown are the three iso-surfaces for both foci, where the combined excitation and detection efficiency for a fluorescence photon has decreased to $1 / e, 1 / e^{2}$, and $1 / e^{3}$ of its maximum value in the center of the focus. 
The results of a 2fFCS measurement in a plane for ideal aberration-free conditions are depicted in figure 25, where the fitted values of the diffusion coeffcients are plotted as a function of the plane's position with respect to the focus' beam waist for different degrees of focusing. For laser beam radii between 1.25 and $2.25 \mathrm{~mm}$, the systematic error between fitted and actual value of the diffusion coefficient remains below $5 \%$ within a range of $\pm 0.5 \mu \mathrm{m}$ around the focal plane. However, this range narrows for tighter focusing which results in large systematic errors when the distance between the plane of diffusing molecules and the focal plane increases. The main reason for this is that for tighter focusing, the transversal excitation intensity profile can no longer be well approximated with a Gaussian distribution. This is, however, the basis of the correlation fit curves. Thus, since high accuracy combined with a low sensitivity for focus placement are advantageous for measurements in membranes, relaxed focusing is the setting of choice. This corresponds to focus beam waists of around $300-400 \mathrm{~nm}$. Moreover, these results indicate that $2 \mathrm{fFCS}$ is robust against vertical membrane fluctuations with amplitudes below 100 - $200 \mathrm{~nm}$ which often occur in GUVs $\underline{45}$.

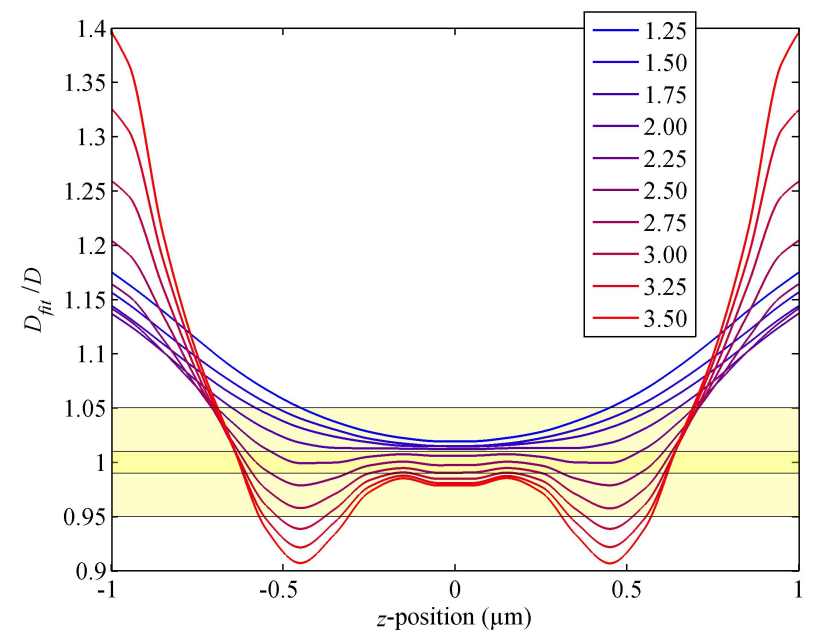

Figure 25: Modeled 2fFCS measurement of diffusion within a plane in an aberration-free system. Shown is the ratio of fitted values to actual values of the diffusion coeffcient as a function of the plane position with respect to the laser beam waist. Results are shown for different degrees of focusing. The legend indicates the radius values of the laser beam coupled into the objective in mm. Shaded areas indicate $1 \%$ and $5 \%$ error margins.

Since aberrations are nearly unavoidable in optical systems, it makes sense to ac- 
count for them as well. The most common causes of aberrations are cover slide thickness deviations and refractive index mismatch between the sample solution and the immersion medium on the objective. Most free-standing bilayer systems operate with a relatively large distance (on the order of $100 \mu \mathrm{m}$ ) between membrane and cover slide. Thus, even small mismatches can accumulate over the long optical path length and cause large aberrations.

To investigate the effect of aberrations caused by refractive index mismatch, we assume that the sample solution has a refractive index of 1.36 while the immersion medium, water, has a refractive index of 1.33 , and that the focal plane of the objective is located $100 \mu \mathrm{m}$ above the cover slide. The computational result for a model 2fFCS measurement at different $z$-positions along the optical axis is shown in figure 26 . Here and hereafter, only the case of relaxed focusing (laser beam radius below $2 \mathrm{~mm}$ ) is considered.

Depicted are the fitted diffusion coefficients with respect to the exact value (top panel), the fluorescence intensities (middle panel) and the molecular brightness (bottom panel) as a function of the $z$-position along the optical axis. The model curves purposefully start at a $z$-position larger than $100 \mu \mathrm{m}$ because in a real experiment, refractive index mismatch causes a displacement of the foci deeper into the solution when positioning them $100 \mu \mathrm{m}$ above the cover slide by moving the objective accordingly. The intensity and molecular brightness can be calculated in the following way. For a fixed molecular concentration (molecules per unit area), the mean number of molecules within the detection area is proportional to

$$
V_{e f f}(z)=\frac{\left[\int d \rho U(\rho, z)\right]^{2}}{\int d \rho U^{2}(\rho, z)}
$$

where $U(\rho, z)$ is a two-dimensional Gaussian distribution. The mean observable fluorescence intensity of one molecule is proportional to

$$
\bar{I}(z)=\int d \rho U(\rho, z)
$$

Therefore, the average observable molecular brightness for molecules diffusing within a plane at position $z$ is proportional to $\bar{I}(z) / V_{\text {eff }}(z)$ which is plotted in the lower panel of figure 26 .

The second main reason for aberrations are cover-slide thickness deviations. Stateof-the-art water immersion objectives are designed to take the presence of a glass cover slide of specific thickness between objective and sample into account. The objective used in our setup has an adjustment ring with which a specific cover slide 


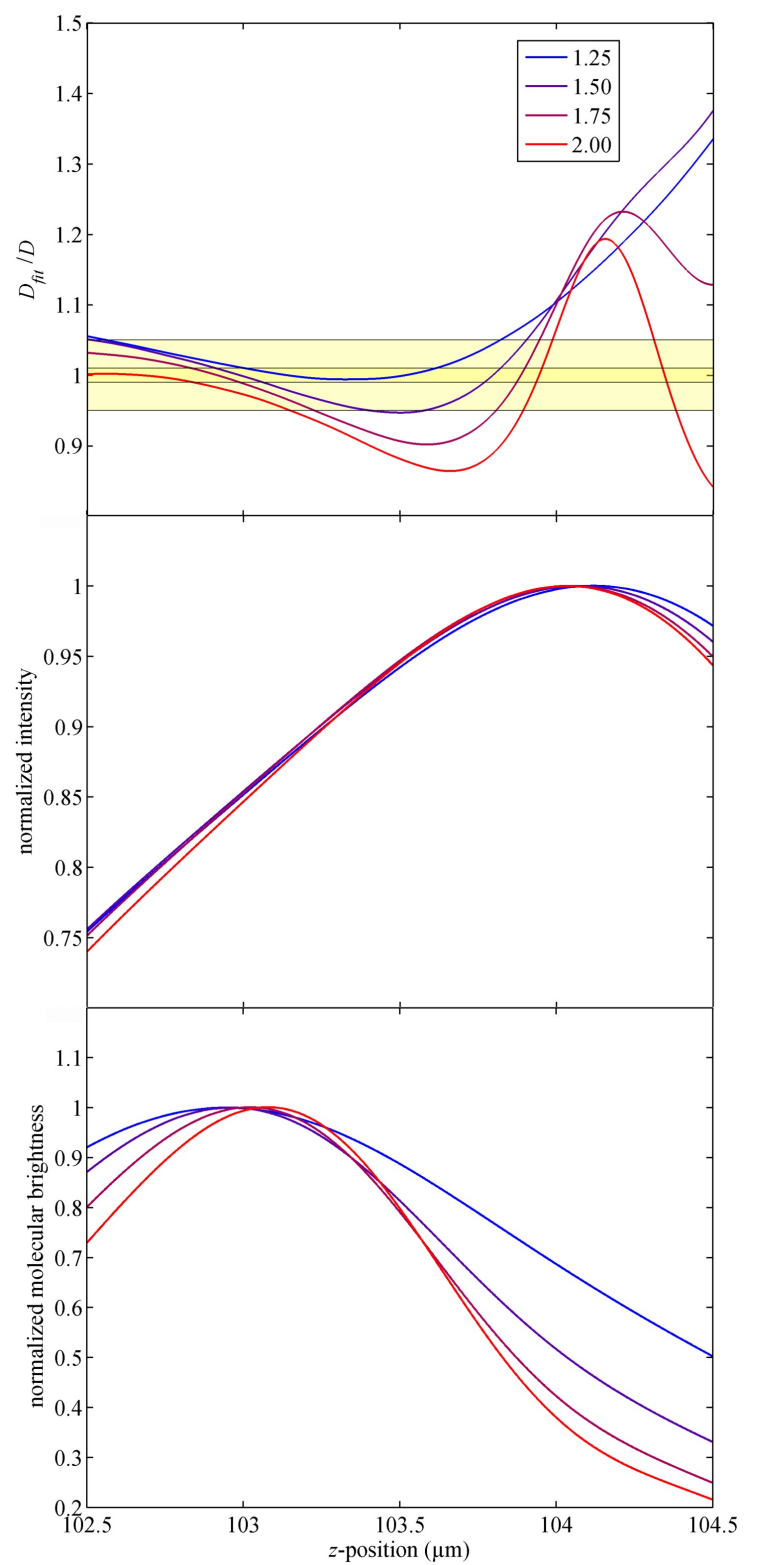

Figure 26: Modeled 2fFCS measurement with aberrations due to refractive index mismatch. Top panel: Fitted diffusion coefficient relative to its exact value. The legend indicates the radius values of the laser beam coupled into the objective in $\mathrm{mm}$. Shaded areas indicate $1 \%$ and $5 \%$ error margins. Middle panel: Mean fluorescence intensity (average over plane). Bottom panel: Molecular brightness. All curves are plotted as a function of the z-position along the optical axis.

thickness can be matched. In practice, however, cover slides rarely have the exact thickness indicated by the supplier, and positioning the ring on the objective is rather unprecise rendering correct adjustment very difficult. Therefore, deviations on the order of $10 \mu \mathrm{m}$ between actual cover slide thickness and the value which the objective 
is adjusted to are mostly unavoidable. Since FCS is extremely sensitive to changes in the size and shape of the MDF, these small deviations already influence the experiment's outcome quite drastically. The result of a model 2fFCS measurement including aberrations caused by cover slide thickness deviations of $10 \mu \mathrm{m}$ is shown in figure 27.

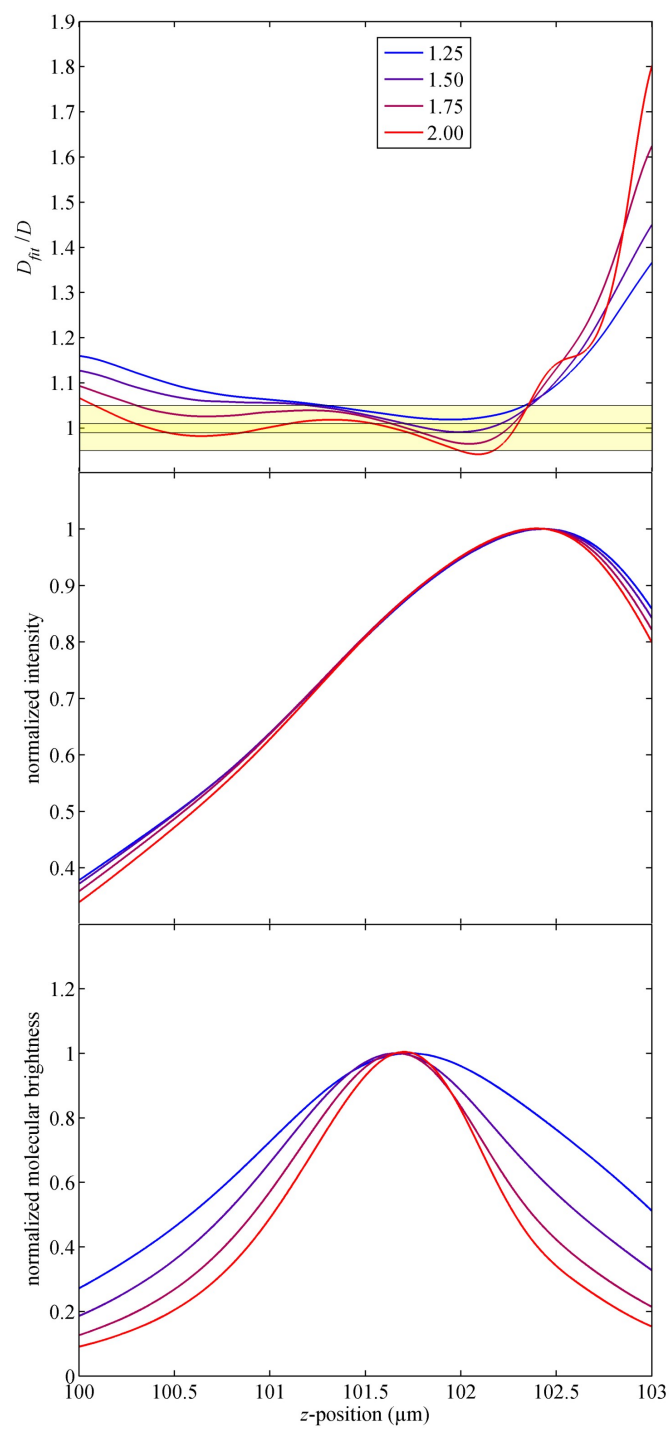

Figure 27: Modeled 2fFCS measurement with aberrations due to cover slide thickness deviations. Top panel: Fitted diffusion coefficient relative to its exact value. The legend indicates the radius values of the laser beam coupled into the objective in $\mathrm{mm}$. Shaded areas indicate $1 \%$ and $5 \%$ error margins. Middle panel: Mean fluorescence intensity (average over plane). Bottom panel: Molecular brightness. All curves are plotted as a function of the z-position along the optical axis.

These modeled measurements including aberrations have an important consequence for real experiments where the laser foci have to be positioned exactly on the bilayer. 
Figure 26 and figure 27 show that the maximum fluorescence intensity does not coincide with the correct position on the membrane, i.e. the region of least systematic error of the fitted diffusion coefficient. The maximum of fluorescence intensity is right-shifted with respect to this region in both cases, regardless of whether the aberrations were caused by refractive index mismatch or cover slide thickness deviations. In both cases, spherical aberrations cause a displacement between the maximum of the detection efficiency and the minimum beam waist of the focused laser beam along the optical axis. As a consequence, the transversal profile of the MDF becomes non-Gaussian very quickly when moving away from the plane of smallest focus diameter. Positioning the foci according to the maximum fluorescence intensity therefore leads to a systematic overestimation of the diffusion coefficient. This problem is not specific to 2fFCS but will also occur in line-scan and scanning-focus FCS. The only exception is $z$-scan FCS which allows for accurate positioning but at the expense of very long measurement times of typically 30 - 40 minutes.

Comparing the top and bottom panels of figure 26 and figure 27, however, shows that the maximum molecular brightness coincides with the area of least systematic error in diffusion coefficients. It therefore provides a very accurate tool for determining the correct focus position on the bilayer. Moreover, this positioning method is very fast (typically 3 - 5 minutes), thus allowing precise positioning in free-standing lipid bilayer systems, i.e. GUVs and BLMs. 
2. Materials And Methods

\subsubsection{2fFCS Measurements}

Lipid diffusion in membranes was measured under different buffer conditions in order to study the effect of mono- and divalent ions in neutral and negatively charged bilayers. Neutral bilayers were generated using a mixture of 60 weight $\%$ POPE and 40 weight\% POPC. Negatively charged bilayers were formed using a POPC/POPE/DOPG mixture with 1 weight\% DOPG. The buffer conditions used are listed in table 1 .

Table 1: Lipid and buffer compositions used for 2fFCS measurements of lipid diffusion.

\begin{tabular}{lcl}
\hline \multicolumn{1}{c}{ Lipid mixture } & $\begin{array}{c}\text { Ion concentration } \\
\text { varied }\end{array}$ & \multicolumn{1}{c}{ Buffer } \\
\hline POPC/POPE & $\mathrm{Ca}^{2+}$ & $50 \mathrm{mM} \mathrm{TRIS}, 150 \mathrm{mM} \mathrm{NaCl}$, \\
& & $5 \mathrm{mM} \mathrm{KCl}$ \\
POPC/POPE & $\mathrm{K}^{+}$ & $50 \mathrm{mM}$ TRIS, $150 \mathrm{mM} \mathrm{NaCl}$, \\
& & $5 \mathrm{mM} \mathrm{KCl}$ \\
POPC/POPE & $\mathrm{Na}^{+}$ & $50 \mathrm{mM}$ TRIS \\
& & $($ except at first point: \\
& & $50 \mathrm{mM}$ TRIS, $5 \mathrm{mM} \mathrm{KCl})$ \\
POPC/POPE/DOPG & $\mathrm{Ca}^{2+}$ & $50 \mathrm{mM}$ TRIS, $150 \mathrm{mM} \mathrm{NaCl}$, \\
& & $5 \mathrm{mM} \mathrm{KCl}$ \\
POPC/POPE/DOPG & $\mathrm{K}^{+}$ & $50 \mathrm{mM}$ TRIS \\
& & $50 \mathrm{mM}$ TRIS \\
\hline
\end{tabular}

For all buffers used, the refractive index has been determined to be 1.34, which is slightly above the refractive index of pure water (1.33). The refractive indices were measured using a refractometer (Carl Zeiss, Jena, Germany) at room temperature. The buffers were prepared by dilution of a stock solution of higher concentration in order to minimize weighing errors especially in the calcium series. Several concentrations of this series $\left(5 \mathrm{mM}, 6 \mathrm{mM}\right.$ and $8 \mathrm{mM} \mathrm{CaCl}_{2}$ ) were also measured with 
buffers made from different stock solutions to quantify the error. The same diffusion coefficients were obtained independent of the stock solution used, indicating that the error is within $5 \%$. An overview of the ion concentrations investigated is given in table 2 .

Table 2: Investigated ion concentrations in lipid diffusion measurements in POPC/POPE and $P O P C / P O P E / D O P G$.

\begin{tabular}{ccc}
\hline Ion & concentration & relative ionic strength \\
\hline $\mathrm{Na}^{+}$ & $c /$ mM & $I_{\text {rel }} / \mathbf{~ m M}$ \\
& 0 & 0 \\
& 3 & 3 \\
& 15 & 15 \\
& 30 & 30 \\
\hline $\mathrm{K}^{+}$ & 0 & 0 \\
& 15 & 15 \\
& 30 & 30 \\
\hline $\mathrm{Ca}^{2+}$ & 0 & 0 \\
& 1 & 3 \\
& 3 & 9 \\
& 5 & 15 \\
6 & 18 \\
& 8 & 24 \\
& 10 & 30 \\
\hline
\end{tabular}

The relative ionic strengths $I_{r e l}$ are listed as well. $I_{r e l}$ describes the difference between the ionic strength of the solution after addition of the respective ions and the ionic strength of the buffers given in table $11 I_{\text {buffer }+ \text { salt }}-I_{\text {buffer }}$.

All protein diffusion measurements in solution and in the membrane, as well as the lipid diffusion measurements for investigating the Saffman-Delbrück model have been performed in PBS ( $\mathrm{pH}$ 7.4). All measurements have been done at $22{ }^{\circ} \mathrm{C}$. Measurement time was always 10 minutes, except when specifically stated otherwise. All measurements were performed at a laser power of $3 \mu \mathrm{W}$ to avoid photobleaching. For measurements of proteins in solution, the respective samples were either diluted 1:100, i.e. below the surfactant's critical micelle concentration (cmc), or the surfactant was removed by size-exclusion chromatography using a Sephadex G-25 column. 
KcsA was relatively stable after surfactant removal and both methods yielded the same results. In contrast, $\mathrm{EcClC}$ and $\mathrm{AcrB}$ were very unstable upon surfactant removal. Size-exclusion chromatography yielded more homogeneous samples for Ec$\mathrm{ClC}$ and $\mathrm{AcrB}$ and was thus performed before 2fFCS measurements in solution. All samples were freshly prepared before each measurement and only used once for a 10-minute measurement. 


\section{Lipid Diffusion in Black Lipid Membranes}

\subsection{Application of the Maximum Molecular Brightness Method for Focus Positioning on the BLM}

Lipid diffusion in BLMs was measured with 2fFCS, which requires precise adjustment of the foci on the bilayer. Therefore, $x / z$-images of the pore and the membrane were taken prior to each measurement (figure 28).

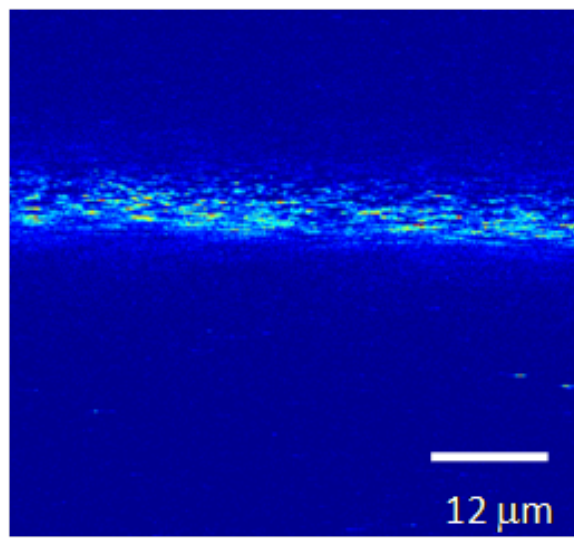

Figure 28: $x / z$-Image of a POPC/POPE BLM with head-group-labeled

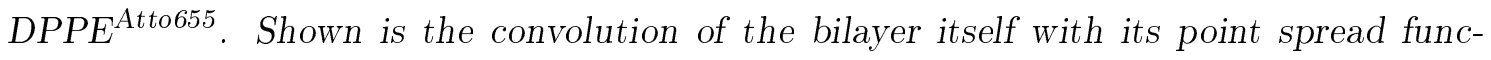
tion which makes it appear broader than the actual bilayer thickness.

As was shown in the previous section, the position of maximum intensity does not necessarily yield correct adjustment of the foci on the bilayer. Instead, the maximum molecular brightness was found to provide a more robust method for positioning. In order to test the applicability of the maximum molecular brightness as a positioning tool and check for aberrations in our system, $z$-scans across the BLM were performed. Start and end point of the scans were chosen from the $x / z$-image (figure 28) to ensure that the membrane is fully covered during the scan. The resulting diffusion coeffcients are depicted in the top panel of figure 29, along with the intensity (middle panel) and the molecular brightness, i.e. the counts per second per molecule (bottom panel).

Since multiple positions had to be covered during the $z$-scan, the measurement time for each point was set to $200 \mathrm{~s}$ in order to avoid mechanical drift of the setup and decrease the risk of bilayer movement or rupture during the experiment. The obtained curves are in good qualitative agreement with the theoretical data presented in figure 27. Therefore, it can be concluded that aberrations are present in the opti- 


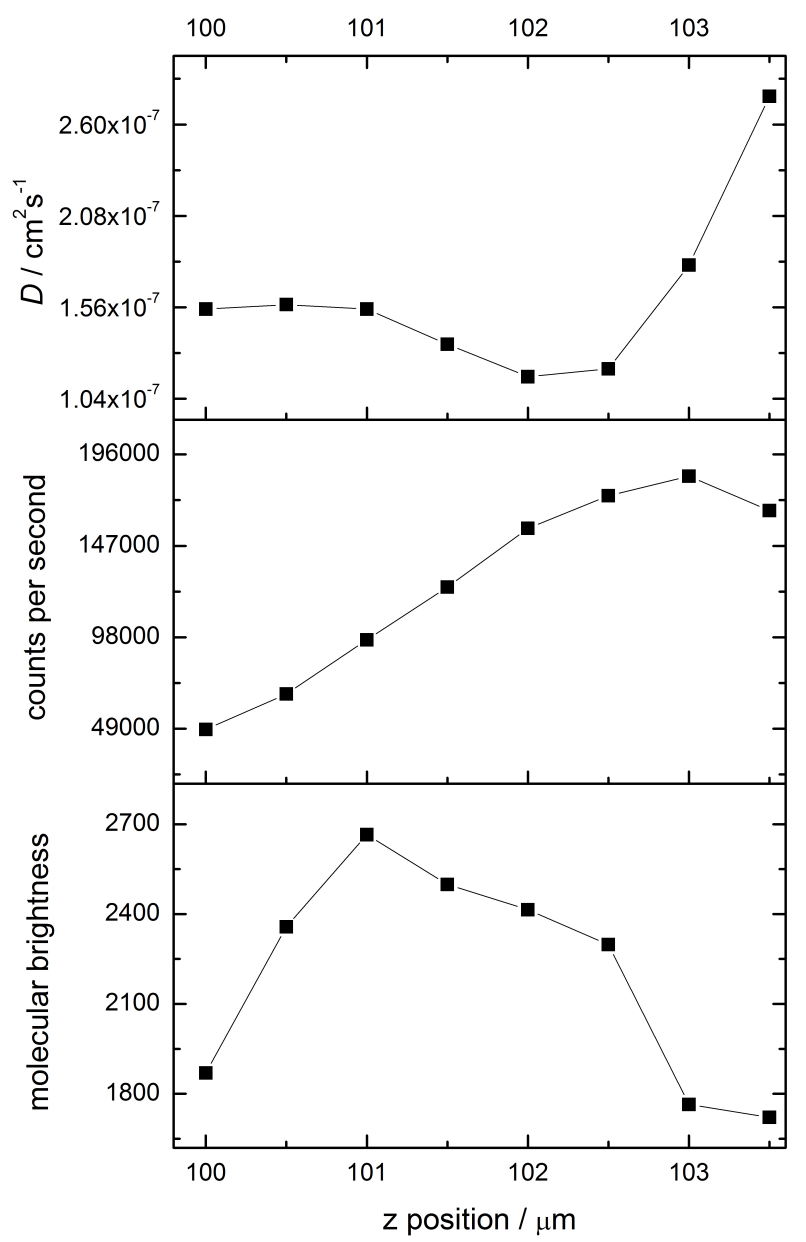

Figure 29: z-Scan across the membrane with 2fFCS. Shown are the diffusion coeffcients (top panel), the fluorescence intensity (middle panel) and the molecular brightness, i.e. the count rate per molecule (bottom panel) as a function of focus position.

cal system. These aberrations are mostly caused by cover slide thickness deviations and are partly due to refractive index mismatch since the used buffer had a slightly higher refractive index (1.34) as compared to pure water (1.33).

Figure 29 illustrates again, that the maximum intensity does not coincide with the correct diffusion coefficient, which corresponds to the minimum of the $D$ vs. $z$ position curve in the top panel. Instead, the maximum molecular brightness corresponds well to the minimum diffusion coefficent and thus provides a fast and robust alternative for precise focus adjustment. The drastic increase in diffusion coefficients between $z=102.5 \mu \mathrm{m}$ and $z=103 \mu \mathrm{m}$ can be attributed to the fact, that the intensity profile becomes non-Gaussian because the bilayer moves out of focus, i.e. the plane of maximum molecular brightness. Since the data evaluation relies on the Gaussian approximation, the resulting fits for ACF and CCF worsen dramatically 
and the obtained values for the diffusion coefficient deviate strongly from its actual value.

These findings show that $2 \mathrm{fFCS}$ can be used to measure diffusion processes in membranes with high accuracy. The maximum molecular brightness provides a robust tool for correct positioning of the foci. This procedure would not work for single focus FCS, since due to optical aberrations, the focus diameter in a specific plane can change from measurement to measurement. Exact knowledge of the MDF and thus the shape of the focus is, however, an essential requirement in single focus FCS. Therefore, severely wrong diffusion coefficients would be obtained with FCS using the maximum molecular brightness for focus adjustment. The only method with comparable performance is $z$-scan FCS, which requires much longer measurement times (about 30-40 minutes) compared to the maximum molecular brightness method (about 3 minutes).

In summary, diffusion measurements in BLMs with 2fFCS can be performed as follows: After acquiring an $x / z$-image of the membrane perpendicular to the bilayer plane, the maximum intensity can be obtained from the image. Short FCS measurements around the position of maximum intensity can then be performed to find the position of maximum molecular brightness, which is used for focus adjustment in the experiment. Since the bilayer can move out of focus or rupture during the experiment, the measurement times have to be kept short (the maximum measurement time in the presented experiments was 10 minutes) and the foci have to be readjusted before each measurement. Bilayer movement out of focus or membrane rupture can be detected in the resulting correlation curves, because they lead to a pronounced decrease in count rate and a loss of correlation. For evaluation, only correlation curves of the intact bilayer in focus are considered. This makes $2 \mathrm{fFCS}$ a robust and highly accurate method even when determining diffusion coefficients in free-standing lipid bilayer systems like GUVs or BLMs and offers the possibility to work also in more complicated systems as well as in live cells.

\subsection{Influence of Solvent within Black Lipid Membranes}

Black Lipid Membranes are generated using the painting technique (described in detail in the introduction), which generally requires the lipids to be dissolved in an organic solvent in order to stably span the membrane over a large pore. This technique thus bears the risk, that small amounts of organic solvent remain within 
the bilayer. Since residual solvent would change the membrane viscosity, it would in turn severely affect the obtained diffuion coefficients. The influence of potentially remaining dodecane inside the BLM was therefore checked by comparing lipid diffusion in the used BLM system with lipid diffusion in solvent-free GUVs.

Lipid diffusion measurements in GUVs were performed in a $3.4 \%$ aqueous sugar (1:1 mixture of glucose and sucrose) solution. The viscosity of this solution was previously determined by measuring Alexa647 succinimidyl ester in the respective sugar mixture and was found to be $0.99 \mathrm{mPa} \cdot \mathrm{s}$ at $22{ }^{\circ} \mathrm{C}$. The obtained viscosity essentially matches the viscosity of pure water $\left(0.96 \mathrm{mPa} \cdot \mathrm{s}\right.$ at $\left.22{ }^{\circ} \mathrm{C}\right)$. Thus, the results for lipid diffuion in GUVs and BLMs can be compared directly.

In BLMs, a lipid diffusion coeffcient of $D_{\mathrm{BLM}}=11.6 \pm 0.6 \mu \mathrm{m}^{2} \mathrm{~s}^{-1}$ was measured. In GUVs, a lipid diffusion coeffcient of $D_{\mathrm{BLM}}=11.5 \pm 0.6 \mu \mathrm{m}^{2} \mathrm{~s}^{-1}$ was obtained. The viscosity-corrected results for BLMs and GUVs match perfectly, indicating that the effect of remaining solvent inside the BLM is negligibly small.

\subsection{Influence of Mono- and Divalent Ions}

2fFCS including the newly developed positioning technique were first applied to study lipid diffusion in membranes. In particular, the effect of mono- and divalent ions on lipid diffusion in neutral and charged bilayers was investigated. A lipid mixture of 60 weight\% POPE and 40 weight\% POPC was used to generate neutral BLMs. These lipids are abundant in biological systems and form a homogeneous bilayer with a phase transition temperature of $22{ }^{\circ} \mathrm{C}$. In order to obtain negatively charged bilayers, 1 weight\% DOPG was added to the POPC/POPE mixture, which also yields a homogeneous membrane. For 2fFCS measurements, fluorescently la-

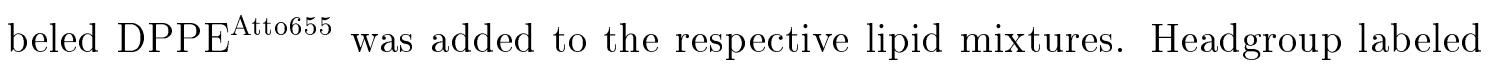
lipids were chosen to avoid any effect of the fluorescent dye on the phase behavior of the lipid mixture or the lipid diffusion. Moreover, only small concentrations of labeled lipids were required for 2fFCS measurements. Addition of DPPE ${ }^{\text {Atto655 in a }}$ molar ratio of 1:4000000 with respect to POPE was found to be sufficient. Slightly higher concentrations of labeled lipids decrease the amplitude of the correlation curves but do not affect the resulting diffusion coefficient. Therefore, addition of small amounts of fluorescently labeled DPPE ${ }^{\text {Atto655 }}$ should not influence the diffusion processes or phase behavior of the respective BLM mixtures.

The obtained auto- and crosscorrelation curves could be fitted well with the twodimensional diffusion model described in the theory section (figure 30. 


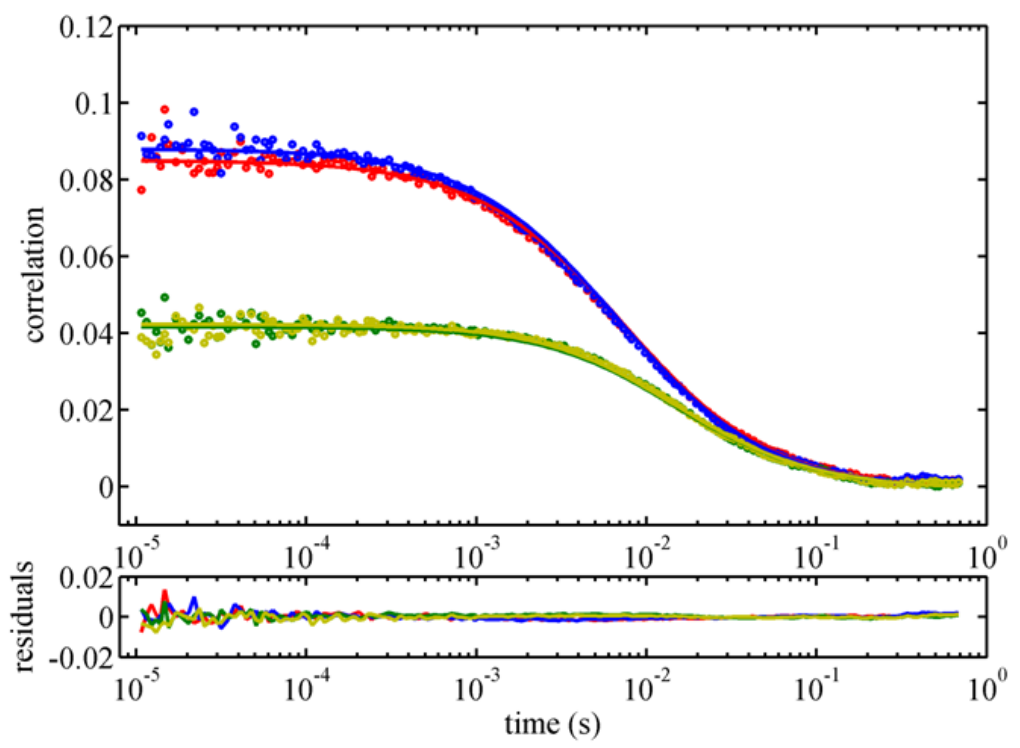

Figure 30: Autocorrelation (red, blue) and crosscorrelation curves (yellow, green) for lipid diffusion of DPPE ${ }^{\text {Atto655 }}$ in a POPC/POPE BLM. Solid lines are fits to the data (circles). The lipid mixture contained $6 \mathrm{mg} / \mathrm{mL} \mathrm{POPE}, 4 \mathrm{mg} / \mathrm{mL}$

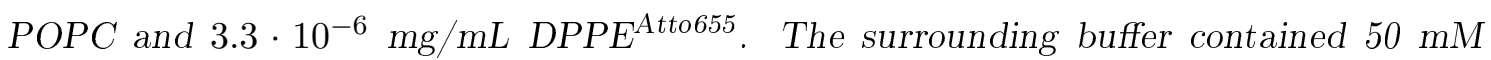
TRIS (pH 7.5), $150 \mathrm{mM} \mathrm{NaCl,} 5 \mathrm{mM} \mathrm{KCl}$ and $3 \mathrm{mM} \mathrm{CaCl}_{2}$

The membranes were generated in buffers of different ionic strengths. Moreover, the concentrations of monovalent sodium and potassium ions and divalent calcium ions were varied. The used buffer compositions are listed in table 1. Whether BLMs were newly formed in the respective buffer or an already existing bilayer was perfused with a buffer of different ionic strength did not influence the obtained diffusion coefficents. In case of BLM perfusion, special care was taken to flush the chip with sufficient amounts of solvent to ensure complete buffer exchange. After perfusion, the system was allowed to equilibrate for 10 minutes.

Each measurement was performed at least three times for 10 minutes. The collected photons were divided into bunches of $10^{6}$ photons per bunch. One measurement yielded on average 60 bunches. If less bunches were obtained during the measurement, e.g. due to bilayer rupture, the measurements were repeated to generate a similar amount of bunches and ensure statistical accuracy. This was necessary, especially at low ionic strengths, since the BLMs were very unstable under these conditions. The obtained diffusion coefficients are shown in figure 31, The respective standard deviation was $5 \%$ or less, except for measurements at very low ionic strength where the error increases due to the instability of the bilayer. 


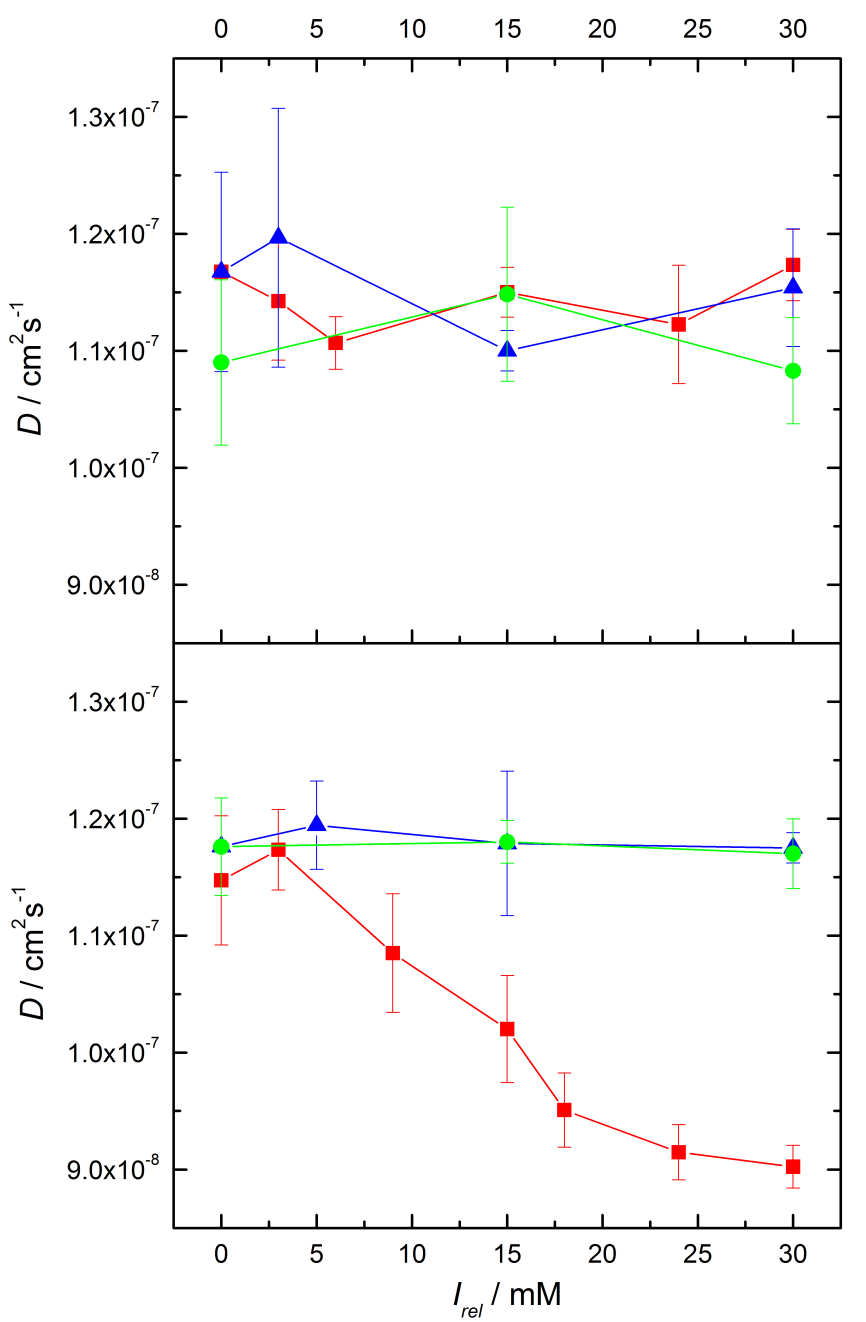

Figure 31: Influence of mono- and divalent cations on lipid diffusion in neutral (top panel) and negatively charged BLMs (bottom panel). Red curves: variation of $\mathrm{CaCl}_{2}$ concentration, blue curves: variation of $\mathrm{KCl}$ concentration, green curves: variation of $\mathrm{NaCl}$ concentration. Buffers used are listed in table 1 .

Overall, variation of the ionic strength in neutral BLM systems did not lead to a systematic change in the lipid's diffusion coefficient (figure 31, top panel). None of the added cations $\left(\mathrm{Ca}^{2+}, \mathrm{K}^{+}, \mathrm{Na}^{+}\right)$affected lipid diffusion in POPC/POPE BLMs. The obtained fluctuations in diffusion coefficients are solely due to membrane instabilities caused by the low ionic strength.

In comparison to neutral BLMs, charged lipid bilayers were much more stable at low ionic strengths and thus less prone to fluctuations. Consequently, the resulting correlation curves were easier to fit and the obtained diffusion coefficients scatter much less. Similar to the case of neutral systems, lipid diffusion in charged POPC/POPE/DOPG membranes remained constant upon variation of the potas- 
sium or sodium concentration up to $30 \mathrm{mM}$ (figure 31, blue and green curves in the lower panel). By comparing these values with the first point of the red curve in the lower panel of figure 31, where lipid diffusion was measured in $50 \mathrm{mM}$ TRIS with $5 \mathrm{mM} \mathrm{KCl}$ and $150 \mathrm{mM} \mathrm{NaCl}$ (see table 1), it can be seen that the diffusion coefficient remains constant even when increasing the sodium concentration to $150 \mathrm{mM}$. Thus, it can be concluded that monovalent ions also do not influence lipid diffusion in charged BLMs. Potassium and sodium ions might be able to penetrate into the bilayer's head group region. This, however, does not seem influence the bilayer structure and viscosity to such an extent (if any) that lipid diffusion is slowed down. This is contradictory to the results obtained in ref. ${ }^{46}$, where conventional singlefocus FCS and molecular dynamics simulations were used to determine lipid diffusion coefficients in supported lipid bilayers. There, a drastic decrease in lipid mobility of over $50 \%$ compared to the original value is reported in a neutral POPC membrane upon addition of $100 \mathrm{mM} \mathrm{NaCl}$. Both, charged and uncharged dyes in the SLB yielded the same result. A decrease in lipid diffusion coefficients of about $20 \%$ upon addition of sodium ions was also observed by Hof et al. ${ }^{47}$. In this study, SLBs consisting of DOPC were investigated using single-focus FCS. Thus, despite using rather similar systems, i.e. SLBs combined with FCS, the results obtained in both studies differ substantially. Therefore, especially with respect to the results reported above for lipid diffusion in free-standing bilayers, the drastic influence of sodium ions on lipid diffusion in questionable.

Both studies use SLBs. This is problematic when studying diffusion processes since the support can severely influence the mobility within the membrane ${ }^{12}$. Compared to our measurements, the obtained diffusion coefficients in ref. ${ }^{47}$ have much larger standard deviations of up to $16 \%$. These large errors can be attributed to the fact that SLB preparation in buffers of low ionic strength is challenging and yields unstable membrane systems. Moreover, only two concentrations (0 mM and $150 \mathrm{mM}$ $\mathrm{NaCl}$ ) were investigated. Considering these experimental details, the influence of sodium ions in ref. ${ }^{47}$ might also be insignificant and therefore agree with the results found in this study. Moreover, the results obtained here are in good agreement with with those found in ref. ${ }^{48}$, where pulsed field gradient NMR was used for diffusion measurements in lipid membranes. There, lipid diffusion was also not influenced by monovalent ions.

In contrast to neutral lipid bilayers, where divalent ions did not have an effect on lipid mobility, diffusion processes in charged lipid bilayers were strongly influenced by 
addition of calcium ions. Lipid diffusion in negatively charged POPC/POPE/DOPG membranes slowed down significantly upon addition of divalent calcium ions. This effect became prominent after addition of $3 \mathrm{mM} \mathrm{CaCl}_{2}$ to the respective buffer which is illustrated in the lower panel of figure 31. A severe decrease in lipid mobillity is observed with increasing calcium concentration between $3 \mathrm{mM}$ and $6 \mathrm{mM} \mathrm{CaCl}$. The curve then flattens between $6 \mathrm{mM}$ and $10 \mathrm{mM} \mathrm{CaCl}_{2}$.

The observed decrease in lipid diffusion coefficients can be attributed to electrostatic interactions between the divalent ions and the negatively charged lipid head groups. The calcium ions are able to bridge the negatively charged lipids together thereby increasing the viscosity of the bilayer. As a consequence, lipid mobility within the membrane is decreased. The flattening of the curve at high calcium concentrations can be explained by a saturation effect. With increasing calcium concentration, more and more lipids will be in complex with calcium ions until at very high concentrations (10 $\mathrm{mM}$ or higher) a large calcium excess is present. Thus, all lipids are bridged and no significant increase in the membrane viscosity is obtained any more.

Overall, the investigated calcium ion concentrations are well above the intracellular calcium concentration of biological cells, which is usually in the nanomolar or, for specific cellular functions, in the micromolar range ${ }^{49}$. This range is entirely covered by the presented data if buffer solutions without and with $1 \mathrm{mM} \mathrm{CaCl}_{2}$ are compared. Extracellular calcium ion concentrations, however, are in the millimolar range ${ }^{49}$. Moreover, the intracellular concentration of magnesium, which is also a divalent ion, ranges between $5-20 \mathrm{mM}[\underline{50}$. Therefore, the observed decrease in lipid diffusion in the investiagted concentration range of divalent cations seems to be physiologically relevant and may well affect lipid diffusion in biological cells. 


\section{Membrane Protein Diffusion in Black Lipid Mem- branes}

The mathematical description of protein diffusion in membranes is challenging and different models have been proposed which vary strongly in their predictions. In particular, the Saffman-Delbrück model finds a logarithmic dependence of the protein's diffusion coefficient on its hydrodynamic radius $\left(D \propto \ln \frac{1}{R}\right)$, whereas Gambin et al. suggested a Stokes-Einstein-like behavior with an inverse proportionality between diffusion coefficient and hydrodynamic radius $\left(D \propto \frac{1}{R}\right)$.

In order to test the validity of these models, the diffusion of lipids and differently sized proteins inside a BLM was investigated with 2fFCS. The same POPC/POPE lipid mixture as above was used, since it yields stable, homogeneous bilayers at $22{ }^{\circ} \mathrm{C}$. Working with homogeneous membrane model systems simplifies the comparison when studying proteins with different structural features. In the homogeneous BLM used for the experiments, membrane viscosity and thickness are assumed to be constant upon protein reconstitution. This allows for directly comparing the resulting lipid and protein diffusion coefficients.

For diffusion measurements, we have chosen proteins which do not aggregate inside the membrane, which cover a broad size range of one order of magniture and which are well-characterized in terms of crystal structure and functionality. In particular, the heme-protein Cytochrome-B5 (CytB5), which is involved in electron transport, the potassium channel KcsA, the chloride channel EcClC, and the multi-drug efflux pump AcrB were investigated. All proteins including their structural information are listed in table 3 .

The cylindrical radii of the proteins' transmembrane domains were estimated from the respective crystal structures. The differences between the radii obtained from cystallization of proteins, the hydrodynamic radii in solution and the cylindrical radii used in the Saffman-Delbrück model are assumed to be negligible in comparison to the experimental error.

For CytB5, all published structures omit the transmembrane domain. However, it is known that it consists of one $\alpha$-helix ${ }^{51}$. The protein's cylindrical radius was thus estimated to be $0.7 \mathrm{~nm}$, i.e. $0.5 \mathrm{~nm}$ for the fixed backbone plus two C-C distances for the side chains.

Two different approaches were chosen to incorporate the proteins into the bilayer: 
Table 3: Investigated lipids and proteins. The estimated radii $R_{\text {Lit }}$ were taken from the respective crystal structures. Diffusion coefficients $D$ were determined by 2 fFCS. All diffusion measurements were performed at $22{ }^{\circ} \mathrm{C}$. The standard deviation of the measurements is given in brackets. For calculating the standard deviation, six to ten 10-minute measurements were performed for each protein and each reconsitution method, generating a minimum of 80 bunches (with 1 million photons per bunch) for each point.

\begin{tabular}{ccccc}
\hline Protein & Structure & $\mathbf{R}_{\mathbf{L i t}} / \mathbf{n m}$ & $\begin{array}{c}\boldsymbol{D} / \mu \mathbf{m}^{2} \mathbf{s}^{-1} \\
\text { direct } \\
\text { addition }\end{array}$ & $\begin{array}{c}\boldsymbol{D} / \mu \mathbf{m}^{2} \mathbf{s}^{-1} \\
\text { SNARE } \\
\text { fusion }\end{array}$ \\
\hline DPPE (Lipid) & monomer & 0.4 & $11.6( \pm 0.6)$ & - \\
Cyt B5 & monomer & 0.7 & $10.2( \pm 0.6)$ & $10.4( \pm 0.8)$ \\
KcsA & monomer & 1.2 & $9.3( \pm 0.5)$ & - \\
& tetramer & 2.4 & - & $7.8( \pm 0.8)$ \\
EcClC & monomer & 1.8 & $8.5( \pm 0.5)$ & - \\
& dimer & 2.8 & - & $7.5( \pm 0.8)$ \\
AcrB & monomer & 2.1 & $8.5( \pm 0.8)$ & - \\
& trimer & 3.6 & - & $7.2( \pm 0.4)$ \\
\hline
\end{tabular}

direct addition of the respective protein solution to the buffer surrounding the membrane, which lead to incorporation of monomers, and SNARE-mediated vesicle fusion, which allowed for reconstitution of oligimeric protein species. The differences in reconstituted protein species is also reflected by the diffusion coefficients obtained for the respective methods as listed in table 3 .

\subsection{Protein Reconstitution via Direct Addition}

In the first reconstitution approach, fluorescently labeled proteins in aqueous buffer were added directly to the chip. Protein incorporation into the BLM was monitored by taking images of the membrane in the $x / z$-direction, i.e. perpendicular to the bilayer plane. The observed fluorescence inside the BLM could be attributed to the incorporated proteins (figure 32 .

Knowledge of the incorporated protein species, i.e. the structure of the protein inside the membrane, is essential for investigating the applicability of different diffusion models, as they all rely on the comparison between the protein's diffusion coefficient and its radius. To determine the oligomeric state of the proteins inside 


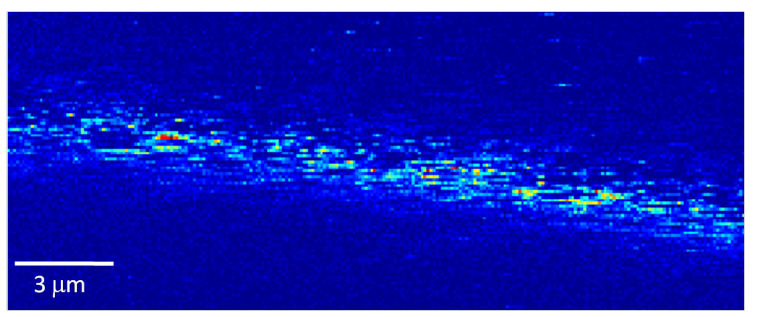

Figure 32: $x / z$-image of $K c s A^{\text {Alexa647 }}$ in POPC/POPE BLM.

the membrane after direct addition, experimental conditions were created which are equivalent to the situation inside the chip prior to protein insertion into the bilayer. Therefore, fluorescently labeled KcsA was first measured in surfactant solution. This corresponds to the protein solution which is added to the chip. These measurements revealed correlation curves which could not be fitted in a meaningful way (figure 33), indicating an extremely polydisperse sample. The reason for this polydispersity is that the proteins are stabilized by surfactant micelles of different size.

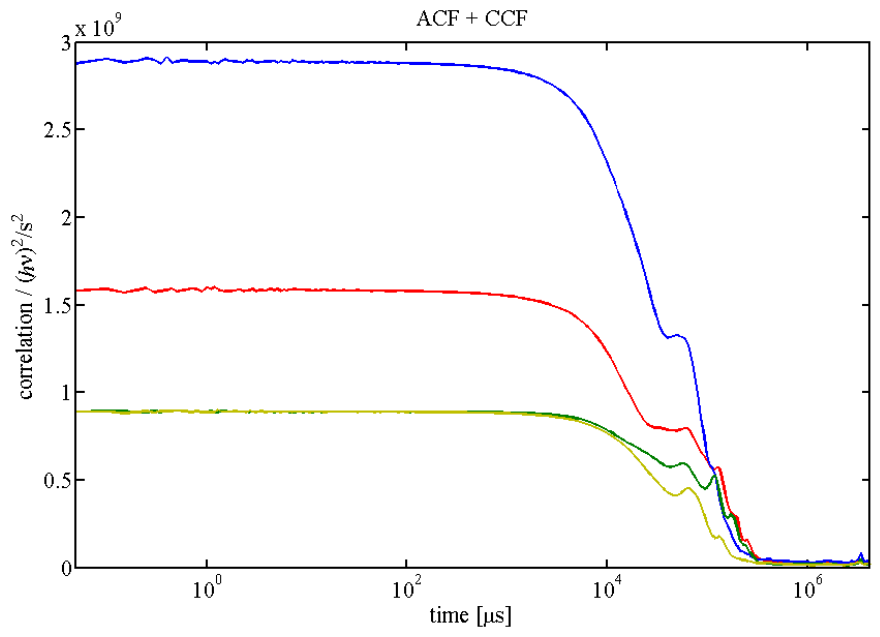

Figure 33: 2fFCS measurement of $\mathrm{KcsA}^{\mathrm{Alexa647}}$ in surfactant solution. Correlation curves indicate an extremely polydisperse sample.

Upon addition to the chip, the protein solution is diluted. The dilution factor is initially about 1:60. During BLM formation, the chip is then flushed with additional solvent (typically at least $1 \mathrm{~mL}$ ) leading to an even higher dilution of the protein solution. The overall dilution factor can thus be estimated to be at least 1:100 prior to protein insertion into the membrane. Therefore, the surfactant concentration inside the chip is decreased well below its cmc of $2 \mathrm{mM}$ during the experiment. Consequently, the KcsA solution was diluted 1:100 and a second 2fFCS measurement in solution was performed. The correlation curves obtained from the diluted sample 
were well-defined and could be fitted perfectly with a single diffusion coefficient (figure 34 indicating a homogeneous, monodisperse solution.

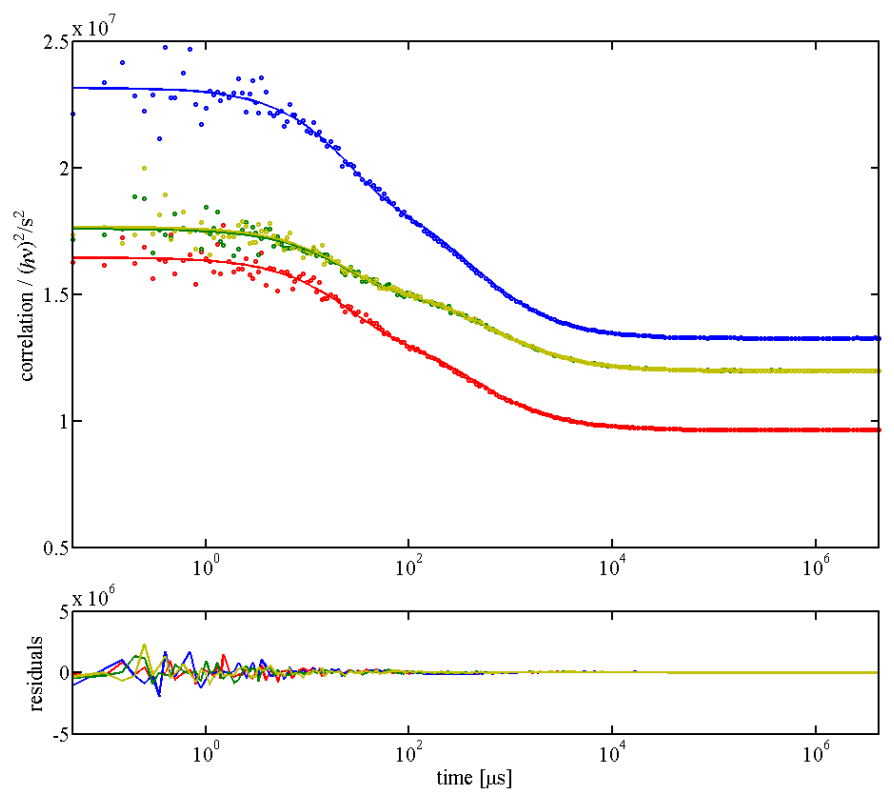

Figure 34: 2fFCS measurement of $\mathrm{Kcs}^{\text {Alexa647 }}$ in solution below the surfactant's cmc. Correlation curves indicate a monodisperse sample. The resulting diffusion coefficient corresponds to KcsA monomer diffusion.

From the measured diffusion coefficient $D$, the (hydrodynamic) radius $R$ of the protein can be calculated using the Stokes-Einstein equation for three-dimensional diffusion

$$
D=\frac{k_{B} T}{6 \pi \mu R}
$$

where $k_{B}$ denotes the Boltzmann constant, $T$ the temperature and $\mu$ the solvent vis$\operatorname{cosity}\left(\mu_{22^{\circ} \mathrm{C}}=0.96 \mathrm{mPa} \cdot \mathrm{s}\right)$. The measurement was performed at room temperature, i.e. $T=295 \mathrm{~K}$. The obtained size of the protein corresponds to the monomeric form of KcsA. The same measurements were performed for EcClC and AcrB, which also yielded diffusion coefficients that indicate the presence of the respective monomers (table 4).

To further confirm the structural state of the proteins within the bilayer, a second control experiment was performed with KcsA. It was previously shown, that this specific potassium channel can disassemble and form monomers upon heating 36 . Therefore, fluorescently labeled KcsA after purification (i.e. in presence of surfactant) was heated following the protocol in ref. ${ }^{36}$. 
Table 4: Protein diffusion in solution after surfactant removal. The radii $R$ were calculated using the Stokes-Einstein equation for three-dimensional diffusion with $T=295 \mathrm{~K}$ and $\mu_{22^{\circ} \mathrm{C}}=0.96 \mathrm{mPa} \cdot \mathrm{s}$ for the aqueous $\mathrm{PBS}$ buffer. The literature values $R_{\text {Lit }}$ were taken from the respective crystal structures.

\begin{tabular}{cccc}
\hline Protein & $\mathbf{R}_{\mathbf{L i t}} / \mathbf{n m}$ & $\boldsymbol{D} / \boldsymbol{\mu m}^{2} \mathbf{s}^{-1}$ & $\boldsymbol{R} / \mathbf{n m}$ \\
\hline $\mathrm{KcsA}$ & 1.2 & $280 \pm 7$ & 0.8 \\
$\mathrm{EcClC}$ & 1.8 & $120 \pm 3$ & 1.9 \\
$\mathrm{AcrB}$ & 2.1 & $107 \pm 6$ & 2.1 \\
\hline
\end{tabular}

The formation of monomers was then shown using sodium dodecyl sulfate polyacrylamide gel electrophoresis (SDS PAGE). The left side of figure 35 shows the SDS PAGE of KcsA after purification without heating, the right side of figure 35 depicts the SDS PAGE of KcsA after heating. The protein is fully converted into its monomeric form after heating as shown in the SDS PAGE.
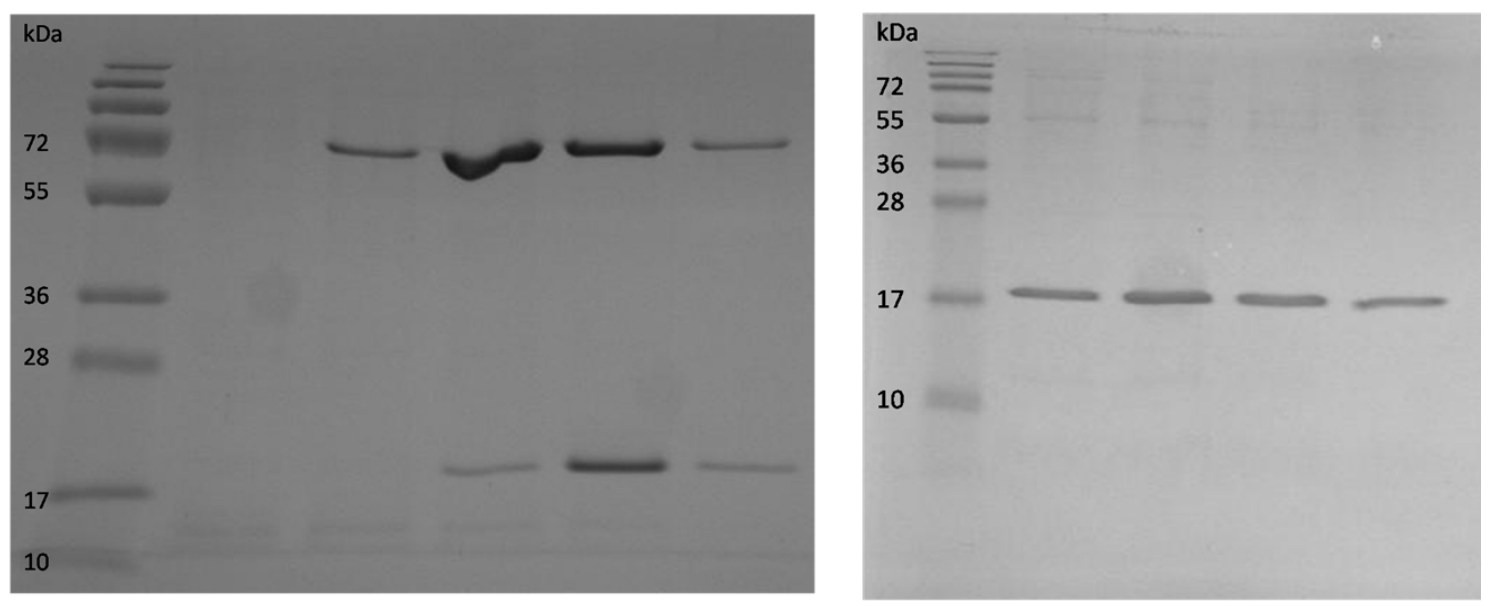

Figure 35: SDS PAGE of KcsA after purification without heating (left) and with heating to $95{ }^{\circ} \mathrm{C}$ for 10 minutes (right). Left lanes in gels show the marker, the other lanes represent the elution fractions from NiNTA purification via His-Tag. Without heating, two bands are observed corresponding to the monomeric $(\approx 18 \mathrm{kDa})$ and tetrameric $(\approx 67 \mathrm{kDa})$ form of KcsA. After heating, only the monomeric from is present.

The monomers generated via heating were then incorporated into a BLM in order to perform 2fFCS and determine their diffusion coefficient within the bilayer. Therefore, the heated KcsA sample was added to the chip. Fluorescence imaging was performed in order to check whether the protein incorporated into the BLM. Since the bilayer was visible and the protein is the only fluorescenly labeled species, the 
fluorescence image revealed that KcsA indeed incorporated into the membrane. The measured diffusion coefficient was $9.1 \pm 0.2 \mu \mathrm{m}^{2} \mathrm{~s}^{-1}$. Without prior heating, a diffusion coefficient of $9.3 \pm 0.5 \mu^{2} \mathrm{~s}^{-1}$ was obtained. An examplary correlation curve is shown in figure 36 .
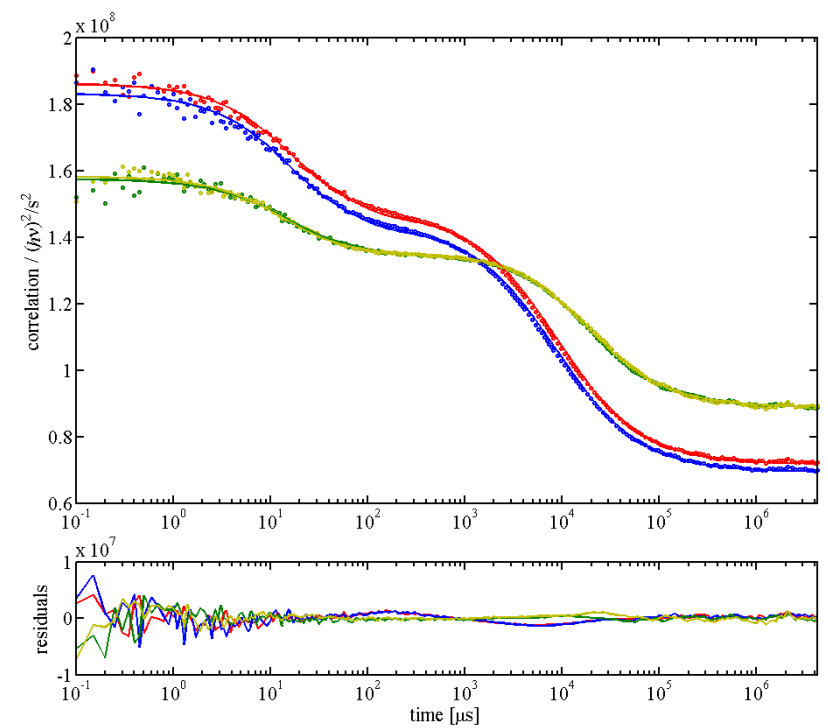

Figure 36: Autocorrelation (blue and red) and crosscorrelation curves (green

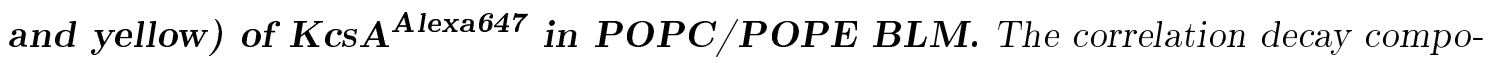
nent at short times is caused by triplet-state photophysics of the dye (Alexa647) and was fitted with an additional exponential term.

The mean values of the diffusion coeffcients obtained with and without heating only differ by $1.4 \%$, which suggests that in both measurements the same species was observed. This leads to the conclusion, that direct addition yields reconstitution of monomers into the bilayer.

\subsection{Protein Reconstitution via SNARE-mediated Vesicle Fu- sion}

The second approach chosen to reconstitute proteins into the bilayer was SNAREmediated vescile fusion. "SNARE" stands for soluble N-ethylmaleimide-sensitivefactor attachment receptor. SNAREs are the key machinery for membrane fusion in eukaryotic cells and seem to mediate membrane fusion in all trafficking steps of the secretory pathway $\underline{52}$. The neuronal SNARE complex consists of three different proteins, Syntaxin-1, SNAP-25 and Synaptobrevin-2, whose primary structures are shown in figure 37 . 

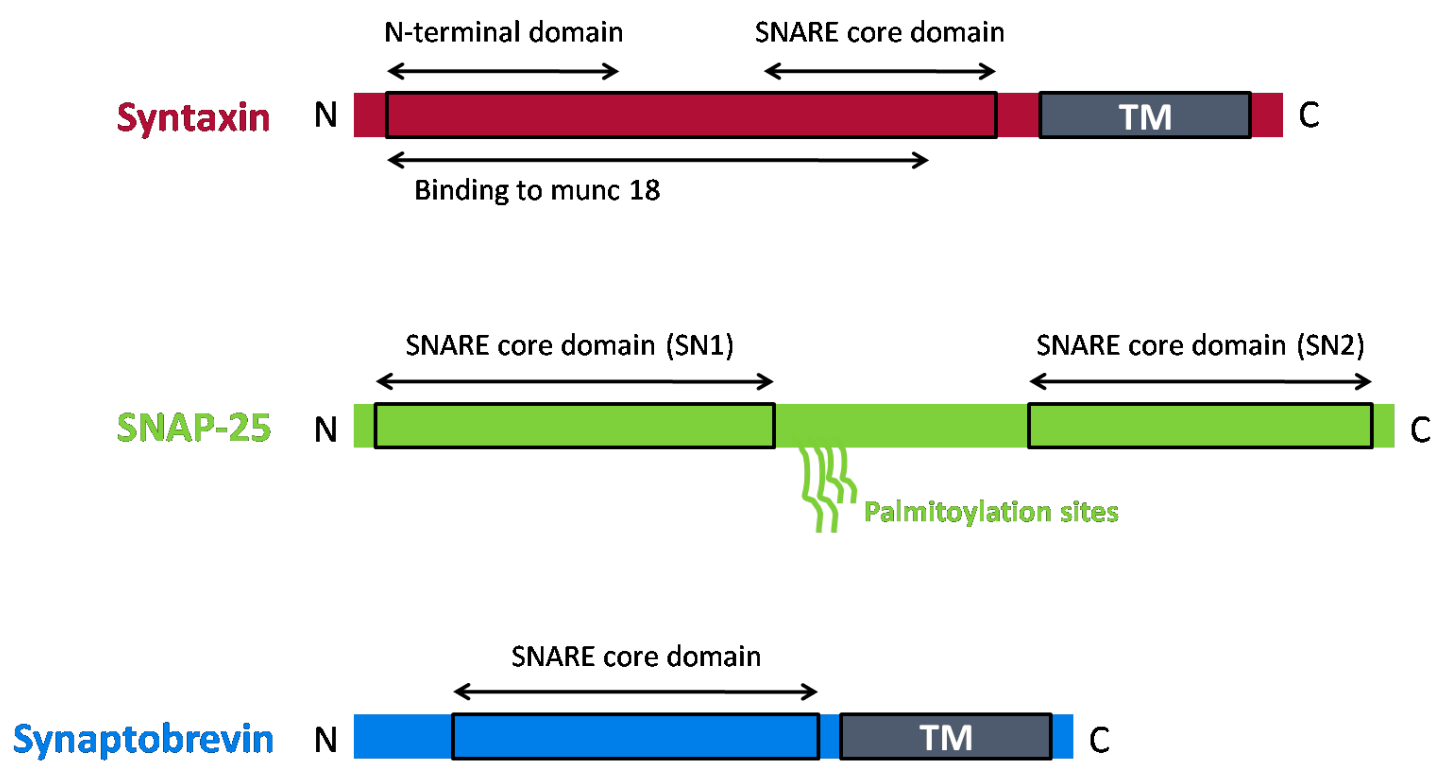

Figure 37: Primary structures of Syntaxin (red), SNAP-23 (green) and Synaptobrevin (blue). Syntaxin-1 used in the present study lacks the $N$-terminal regulatory Habc domain. Figure adapted from ref. $[53]$.

Syntaxin-1 and Synaptobrevin-2 are integral membrane proteins and thus contain a transmembrane domain. SNAP-25 binds to the respective SNARE core domains outside of the membranes forming a four-helix bundle which is illustrated in figure 38 .

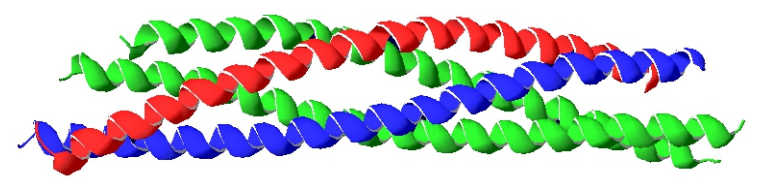

Figure 38: X-ray crystal structure of the core of the neuronal SNARE complex with Syntaxin (red), SNAP-25 (green) and Synaptobrevin (blue) (upper part). Structure represents the cis state, i.e. the fully folded state after fusion. Structure taken from the RCSB protein data bank (1URQ).

In order to allow for fusion, the SNARE proteins have to be localized in opposing membranes. The driving force for membrane fusion is the formation of a fourhelix bundle which leads to a release of free energy. The complex formation tightly connects the two membranes and initiates fusion which is illustrated in figure 39 . Afterwards, SNARE complexes can be "recycled" through dissociation which is mediated by specific proteins called N-ethylmaleimide-sensitive factor (NF) in vivo ${ }^{52}$. In our in vitro system, the SNARE complexes remain bound after membrane fusion. 


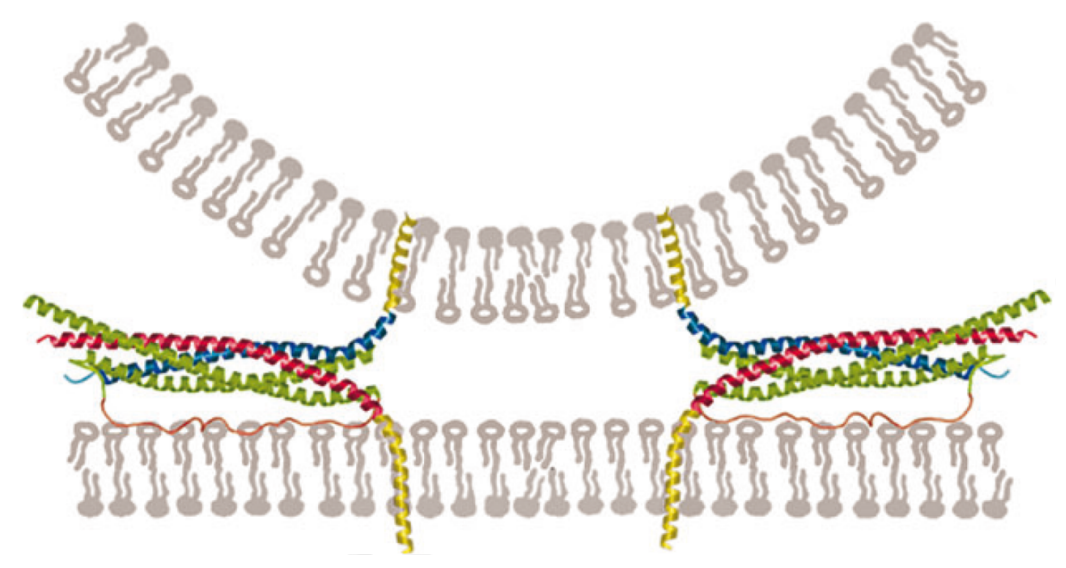

Figure 39: Model of two SNARE complexes in the trans state, which dock a liposome to a SLB in vitro. Figure taken from ref. [53].

For reconstitution, the fluorescently labeled protein of interest was incorporated into large unilamellar vesicles (LUVs) together with the SNARE-protein Synaptobrevin-2 (Syb). $\Delta \mathrm{N}$ complexes, consisting of the SNARE-proteins Syntaxin-1A residues 183288 and SNAP-25, were incorporated into the BLM. Binding Syntaxin and SNAP-25 together prior to fusion leads to a significant increase in fusion speed as compared to regular SNARE-complexes, where SNAP25 and Syntaxin are not previously bound. Fusion of Synaptobrevin with the $\Delta \mathrm{N}$ complex proceeds within 1-2 minutes 34 .

The structure of membrane proteins reconstituted using SNARE-mediated vesicle fusion was exemplary investigated for KcsA using electrophysiology measurements. KesA was chosen because it provides larger currents and longer opening times compared to EcClC. Therefore, electrophysiolgy measurements of KcsA are easier to perform since the channel openings and the resulting conductance steps can be observed even in the presence of relatively high background noise.

Another advantage of $\mathrm{KcsA}$ is that the tetramer forms one transmembrane pore, i.e. the channel is only functional in its fully assembled form. EcClC, however, is a dimer consisting of two separate pores. It has been suggested that these pores function independently, even in monomeric $\mathrm{EcClC}$ [5455]. Thus, distinguishing EcClC monomers and dimers by means of electrophysiology would be much more difficult than distinguishing KcsA monomers and tetramers. AcrB is a multi-drug efflux pump which only works in complex with two other proteins, AcrA and TolC 56. Therefore, its functionality could not be investigated by means of electrophysiology with the experimental setup used in this study. 
Electrophysiology measurements of KcsA were performed in a BLM consisting of POPC/POPE and additionally $1 \mathrm{mg} / \mathrm{mL}$ DOPG, since KcsA gating requires the presence of negatively charged lipids ${ }^{[36}$. To ensure that the addition of negatively charged lipids does not influence protein mobility, KcsA diffusion in a POPC/POPE/ DOPG membrane was measured with 2fFCS as a control experiment. Diffusion coefficients of KcsA in both lipid mixtures were found to be identical. KcsA gating also required PBS buffer of $\mathrm{pH} 4$ containing $400 \mathrm{mM} \mathrm{KCl}$ on both sides of the membrane. Channel opening could be detected using $-200 \mathrm{mV}$ and $+200 \mathrm{mV}$ pulses (figure 40).

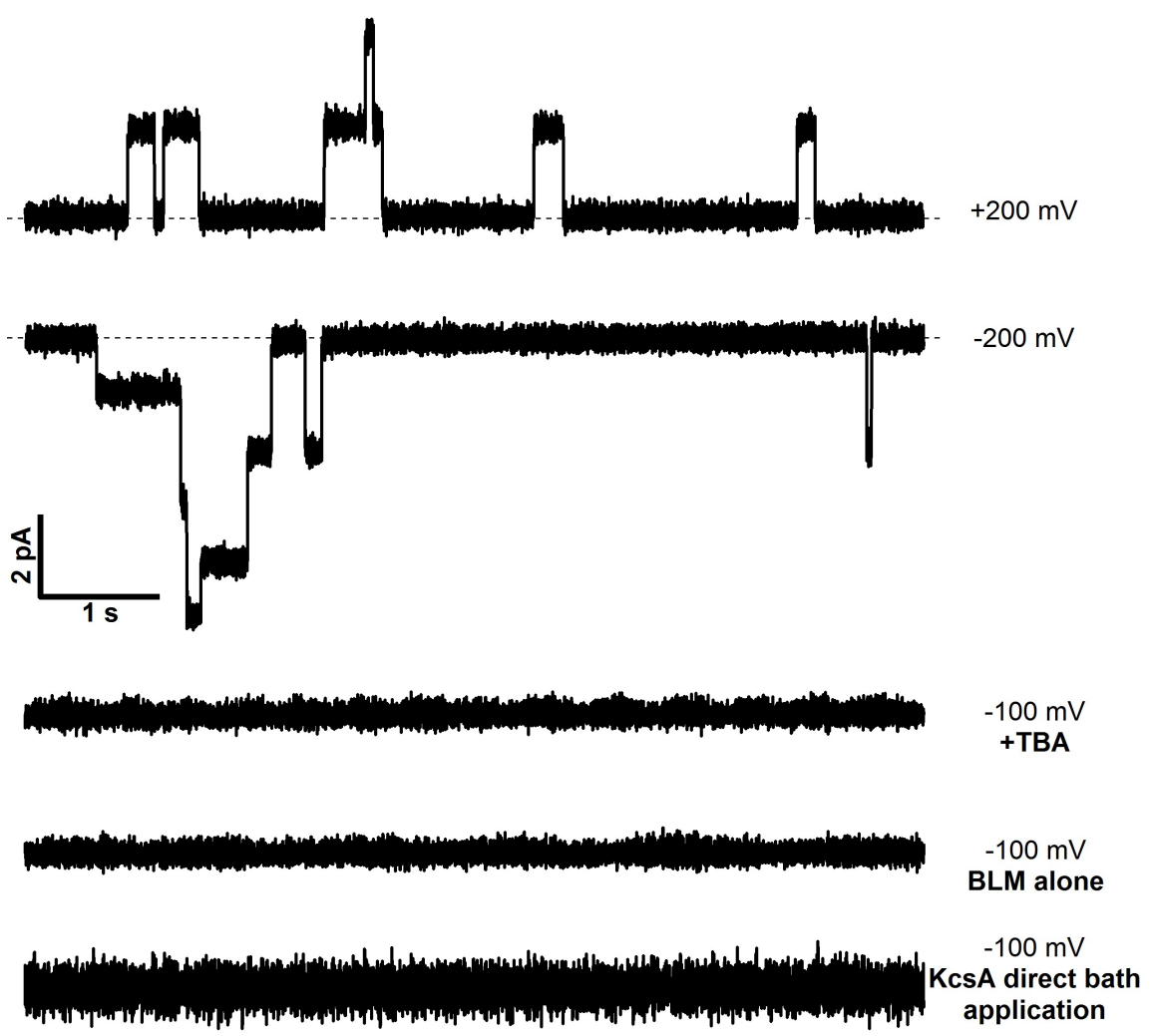

Figure 40: Electrophysiology measurements of KcsA in POPC/POPE/DOPG bilayer. PBS ( $\mathrm{pH}$ 4) with $400 \mathrm{mM} \mathrm{KCl}$ was used as buffer solution. Top: KcsA (tetramer) incorporated via SNARE-mediated vesicle fusion. Bottom: Control experiments. KcsA openings blocked with TBA, BLM without protein and KcsA (monomer) reconstituted via direct addition.

On average, $2.6 \mathrm{pA}$ and $2.3 \mathrm{pA}$ were detected per burst at $-200 \mathrm{mV}$ and $+200 \mathrm{mV}$, respectively. Moreover, a sub-conductance state was measured which is known to exist for $\mathrm{KcsA}[57$ and which was about half the conductance. There are no conductance values published for the exact conditions used in our experiment. While the published data suggest larger values ${ }^{35137}$, it has also been shown that sodium 
ions can partially block KcsA channels and decrease the measured conductance 35 . Since we are working in PBS buffer which contains large amounts of sodium ions, it is likely that the channels in our experiment are also partially blocked. Thus, it can be concluded that the obtained data is consistent with the studies previoulsy published.

To ensure that the observed conductance steps can be attributed to functional KcsA channels within the BLM, several control experiments were performed. First of all, the potassium channels were blocked using tetra-butyl ammonium (TBA) $\stackrel{37}{\text {. TBA }}$ was added to a final concentration of approximately $0.08 \mathrm{mM}$. Upon addition, the conductance steps vanished completely. Secondly, the bilayer was measured without any incorporated proteins to confirm that the conductance steps are due to KcsA gating and do not correspond to any artifacts. The membrane itself did not yield any conductance steps showing that the observed gating is actually due to KcsA incorporation. Finally electrophysiology measurements of directly added KcsA were performed which also did not yield any conductance steps. Therefore, no functional KcsA channels are present in the bilayer upon direct addition.

\subsection{Influence of the Reconstitution Method on the Mecha- nism of Membrane Insertion}

The results obtained in the previous sections suggest that direct addition leads to incorporation of the monomeric form, while SNARE-mediated vesicle fusion yields incorporation of the oligomeric form of the respective proteins. These differences in reconstitution behavior can be attributed to the differences in experimental conditions, in particular the surfactant concentration.

When directly added to the chip, the protein solution is diluted below the surfactant's cmc. As a consequence, the proteins are not stabilized by surfactant micelles anymore. Removal of the surfactant micelles and the resulting destabilization of the protein oligomers leads to the disassembly of proteins into their monomeric form. It is unlikely that all surfactant molecules are removed from the protein surface due to the strong hydrophobic interactions between the surfactant tails with the protein's transmembrane domains. Thus, it is hypothesized that upon dilution, a few surfactant molecules remain attached to the proteins. These surfactant molecules are able to stabilize the protein monomers in solution for a short period of time. This time-span seems to be sufficient for the monomers to insert into the lipid bilayer. The protein-lipid interactions inside the membrane should be much more favorable 
than the interactions with single surfactant molecules, thus resulting in monomer incorporation into the BLM while releasing the remaining surfactant molecules into the surrounding buffer solution.

This theory is supported by protein diffusion measurements in solution before and after dilution below the surfactant's cmc. Before dilution, the sample is polydisperse because the proteins are stabilized by micelles of different size. After dilution, the micelles are destroyed and the sample becomes monodisperse. The obtained diffusion coefficients correspond to the radii of monomers, which indicates that the surfactants remaining on the proteins are closely attached and do not change their size significantly. After monomer incorportation into the bilayer, the proteins could theoretically reassemble. This was, however, not observed in our system which could be attributed to the extremely small concentrations of about one protein per $\mu \mathrm{m}^{2}$.

In contrast, SNARE-mediated vesicle fusion leads to incorporation of proteins in their oligimeric form, because they are highly stabilized during the entire experiment. This is accomplished either by the presence of surfactant micelles or by lipid bilayers (i.e. LUVs or BLMs) upon surfactant removal. The incorporation of proteins into LUVs prior to membrane fusion proceeds at much higher concentrations (in the $\mu \mathrm{M}$ range) compared to the actual measurement and the direct addition pathway (nM to $\mathrm{pM}$ ). Therefore, disassembly of the protein oligomers is unlikely.

For CytB5, the results upon direct addition and SNARE-mediated vesicle fusion are identical, because this protein functionally only exists in the monomeric form. Moreover, CytB5 is purified in a surfactant-free manner, meaning that it is stable in solution before insertion into the membrane and thus does not aggregate or denature.

\subsection{Models for describing Protein Diffusion in Membranes}

Using 2fFCS for measuring protein diffusion in membranes yielded highly accurate quantitative results for the diffusion coeffcients. The obtained diffusion data was plotted against the cylindrical protein radii and fitted with the classical SaffmanDelbrück model (equation 1), the Stokes-Einstein-like model suggested by Gambin et al. (equation 2), and the HPW-based model developed by Petrov and Schwille (equation 3). The fit parameter for all models is the product of membrane viscosity and membrane height $\mu_{m} h$. The result is shown in figure 41 .

The obtained diffusion data is almost perfectly described by the logarithmic scal- 


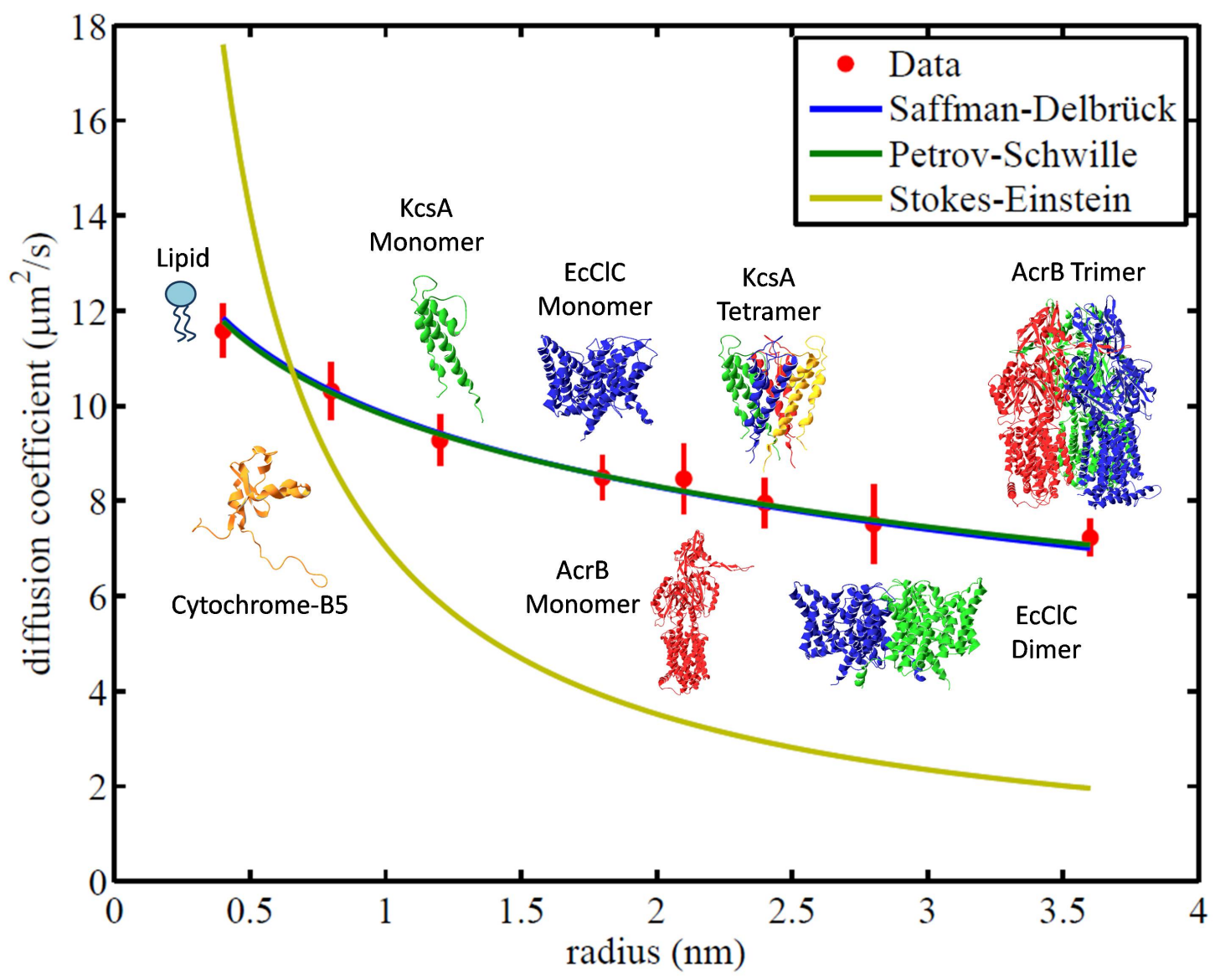

Figure 41: Saffman-Delbrück vs. Stokes-Einstein model. The investigated species are the lipid DPPE and the proteins Cytochrome B5 (depicted without transmembrane domain), KcsA, EcClC and AcrB. The monomeric forms of the proteins were reconstituted via direct addition. The protein oligomers were incorporated into the POPC/POPE BLM via SNARE-mediated vesicle fusion. DPPE was labeled with Atto655, all proteins were labeled with Alexa647. Additionally the HPW-based model by Petrov and Schwille [4] was fitted, which reproduces the classical Saffman-Delbrück result within the size range investigated. The fit parameter for all models was the product of membrane viscosity and thickness $\mu_{m} h$

ing proposed by the Saffman-Delbrück model. In contrast, the linear $1 / R$-scaling suggested by Gambin et al. fails completely in describing protein diffusion in membranes. As expected and previously pointed out theoretically ${ }^{4}$, the HPW-based model reproduces the classical Saffman-Delbrück result for the investigated protein radii. Thus, we can conclude that, despite its simplicity, the Saffman-Delbrück is able to describe protein diffusion in membranes in the studied size range. 
It was shown that for larger membrane inclusions, the logarithmic scaling $\left(D \propto \ln \frac{1}{R}\right)$ becomes invalid and a linear $D \propto \frac{1}{R}$ scaling occurs ${ }^{58}$. The crossover from $D \propto \ln \frac{1}{R}$ to $D \propto \frac{1}{R}$ is determined by the reduced radius $\epsilon^{\prime}$ which is defined as

$$
\epsilon^{\prime}=\frac{R \cdot 2 \mu_{s}}{\mu_{m} h}
$$

with the protein radius $R$, the solvent viscosity $\mu_{s}$, the membrane viscosity $\mu_{m}$ and the membrane thickness $h$. The Saffman-Delbrück model is valid for $\epsilon^{\prime}<0.1$, while it fails completely for $\epsilon^{\prime}>1^{4}$. For the investigated system, the Saffman-Delbrück model is thus valid up to a protein radius of $R=8 \mathrm{~nm}$. This crossover radius can be calculated by inserting the system specific parameters $\mu_{s}=0.96 \mathrm{mPa} \cdot \mathrm{s}$, $\mu_{m}=39.5 \mathrm{mPa} \cdot \mathrm{s}, h=3.8 \mathrm{~nm}$ and $\epsilon^{\prime}=0.1$ into equation 21. The crossover radius is much larger than the investigated protein radii. Therefore, the Saffman-Delbrück model indeed yields an accurate description of protein diffusion in this study.

Whereas the values for the measured diffusion coefficients are very precise, deviations might occur in the radii since the radius values were taken from protein crystal structures and might not match the hydrodynamic or cylindrical protein radii exactly. This should, however, be a minor concern because even relatively large deviations in $R$ of up to $0.5 \mathrm{~nm}$ in either direction would not influence the scaling behavior significantly.

Due to the high accuracy of the diffusion data, the Saffman-Delbrück model can be used to calculate membrane viscosities. Assuming a bilayer thickness of $3.8 \mathrm{~nm} \stackrel{59}{\text {, a }}$ viscosity of $39.5 \mathrm{mPa} \cdot \mathrm{s}$ is obtained from the Saffman-Delbrück equation (equation 1) with $T=295 \mathrm{~K}$. This value is smaller compared to the membrane viscosities reported, which range from 75 to $150 \mathrm{mPa} \cdot \mathrm{s}$. This discrepancy could be explained by differences in the used lipid mixtures and measurement techniques. It can be ruled out that the smaller membrane viscosity obtained in our system is due to residual organic solvent inside the BLM, because exactly the same diffusion coefficient was measured in painted BLMs and solvent-free GUVs prepared with the same lipid mixtures.

A more recent study 61 found membrane viscosities ranging from 3 - $150 \mathrm{mPa} \cdot \mathrm{s}$ in the liquid-disordered phase of domain forming lipid mixtures. The result obtained from the Saffman-Delbrück model fits well into this range.

The obtained diffusion coefficients in this study show a large discrepancy in com- 
parison with the study by Ramadurai et al. ${ }^{23}$. The values found by Ramadurai et al. are about $45 \%$ smaller than the diffusion coefficients measured with $2 \mathrm{fFCS}$. In contrast, their suggested lipid diffusion coefficient of $11.4 \pm 0.7 \mu \mathrm{m}^{2} \mathrm{~s}^{-1}$ corresponds well with the value obtained in this study. However, this makes their lipid diffusion coefficient about twice as high as the diffusion coefficient of the smallest investigated protein Synaptobrevin-2 (Syb), while the difference in radii between lipids and Syb is only $0.1 \mathrm{~nm}$. These deviations might be caused by aggregation of the proteins inside the membrane. This is likely, because GUVs were prepared by drying and rehydration. The respective proteins were therefore reconstituted into LUVs which, after addition of detergent, were dried. This process is inherently accompanied by a change in membrane phases from liquid-disordered $\left(\mathrm{L}_{\mathrm{D}}\right)$ of LUVs in solution to liquid-ordered $\left(\mathrm{L}_{\mathrm{O}}\right)$ in the dried lipid film. This phase transition leads to hydrophobic mismatch between proteins and bilayer in the lipid film. Upon rehydration of the lipid film with an aqueous buffer, GUVs form spontaneously due to electrostatic repulsion between the negatively charged lipids used. Sucrose is added to the aqueous buffer to prevent aggregation of proteins. Tuning the sucrose concentration exactly, however, is absolutely essential. Too much sucrose will prevent GUV formation because the sucrose molecules form a hydration shell around the lipids and would thus prevent the necessary phase transition from liquid-ordered $\left(\mathrm{L}_{\mathrm{O}}\right)$ back to liquiddisordered $\left(\mathrm{L}_{\mathrm{D}}\right)$. If the sucrose concentration is too low, the proteins will aggregate inside the membrane. Precise adjustment of these concentration is therefore crucial but experimentally challenging. Taking this GUV preparation method into account, aggregation of the proteins within the membrane seems likely, which would explain the small values of the obtained diffusion coefficients.

Additionally, the measured diffusion coefficients for larger proteins scatter significantly and the Saffman-Delbrück scaling is only indicated by comparing these values with the diffusion coefficients of Syb and WALP23. Lipid diffusion has been neglected completely. Comparing protein to lipid mobility in these measurements strongly points towards protein aggregation problems during preparation.

Much care has been taken to avoid aggregation in the present study. Proteins were reconstituted either directly or via SNARE-mediated vesicle fusion into the BLM. Both processes proceed without drying or rehydration in the presence of proteins. Another problem in the Ramadurai study could be the use of single-focus FCS, which is extremely sensitive to optical imperfections such as refractive index mismatch, laser beam astigmatism, or cover slide thickness deviations. Absolute values for the diffusion coefficient can only be obtained by calibrating the FCS system with a dye 
of known size and diffusion coefficient, which can be problematic. Moreover, precise alignment of the focal plane with the GUV is challenging.

In contrast, 2fFCS provides a much more robust and accurate tool for diffusion measurements in membranes. Using BLMs instead of GUVs provides a more stable membrane system and allows for working at physiological conditions. Focus positioning with the maximum molecular brightness instead of performing $z$-scans yields a much faster and more accurate alingment method, especially when taking into account that GUVs are much more prone to fluctuations compared to BLMs. Thus, the diffusion coefficients obtained here have a higher accuracy. The measured data nicely match the radii of the investigated species, including lipid diffusion. 


\section{Conclusions \& Outlook}

In this thesis, lipid and protein diffusion in Black Lipid Membranes was investigated using 2fFCS. In order to achieve accurate results, correct positioning of the foci on the membrane is absolutely essential. The maximum molecular brightness was found to be a fast and robust method for finding the correct focus position. It is about ten times faster than $z$-scan FCS and is therefore an attractive alternative for positioning, especially in systems such as BLMs and GUVs.

This newly developed positioning method was used to measure lipid diffusion in neutral POPC/POPE and negatively charged POPC/POPE/DOPG BLMs. Moreover, the effect of monovalent sodium and potassium ions as well as divalent calcium ions on lipid diffusion was investigated. It was shown, that monovalent ions do not influence lipid diffusion regardless of whether the membrane is neutral or charged. The resulting diffusion coefficients in both bilayers were simlar.

Calcium ions do not influence the diffusion in neutral membranes. Addition of $\mathrm{Ca}^{2+}$ to negatively charged bilayers, however, decreases lipid diffusion significantly because calcium ions can link two negatively charged lipids together, thereby increasing the membrane viscosity.

Combining 2fFCS with a Black Lipid Membrane system also allowed for highly accurate measurements of protein diffusion in lipid bilayers. In order to characterize protein diffusion in membranes, we investigated the applicability of the SaffmanDelbrück model ${ }^{1]}$, the Stokes-Einstein-like model suggested by Gambin et al. ${ }^{2,}$, and the HPW-based model dervied by Petrov and Schwille ${ }^{4}$. Therefore, proteins of different sizes were reconstituted into the bilayer. The covered size range of protein radii was one order of magnitude.

Comparing the obtained diffusion coefficients, a $D \propto \ln 1 / R$ scaling is observed. The Saffman-Delbrück model is therefore able to describe diffusion of proteins of various sizes and shapes despite its simplicity. Our measurements also show that the HPW-based model reproduces the Saffman-Delbrück results in the size range investigated. This confirms the theoretical study previously performed by Petrov and Schwille 4 .

After validating the Saffman-Delbrück model, it could be used to precisely determine the viscosity of the lipid membrane, which has been challenging before. We obtained a membrane viscosity of $39.5 \mathrm{mPa} \cdot \mathrm{s}$ for the used POPC/POPE mixture. 
The established technique allows for investigating several aspects of lipid-protein interactions and membrane characteristics, which were previously difficult to study. First of all, the influence of lipid size and charge on protein diffusion can be investigated by varying the lipid composition. Moreover, the buffer composition of the surrounding medium can be varied, in particular the concentrations of mono- and divalent ions, to study not only their effect on lipid diffusion but also on protein diffusion further. This approach could finally lead to reconstitution of proteins into more complicated lipid systems which do not only contain phospholipids but also sphingolipids and cholesterol. In this way, the model membrane systems can be tailored to mimic biological membranes more closely. As opposed to the model systems used so far, biological membranes are highly crowded environments. Preparing membranes including high concentrations of proteins to generate a crowded environment will be the next step in approaching real biological systems in vitro.

Another aspect in studying lipid-protein interactions is the co-diffusion of lipids and proteins in membranes. Thus far, it is not clear whether proteins diffuse by themselves or whether there is a "dragging effect", i.e. the protein is surrounded by a small lipid shell which co-diffuses with the respective protein of interest. The size of the lipid shell could be influenced by the protein's size and shape, since these would directly influence the amount of interactions between proteins and lipids. In this respect, it would also be interesting to consider hydrophobic mismatch between proteins and lipids, since this effect might well influence the size of a co-diffusing lipid shell.

In order to investigate this co-diffusion, Dual-color Fluorescence Crosscorrelation Spectroscopy (2cFCCS) is the method of choice. In 2cFCCS, lipids and proteins are labeled with two different dyes which can be excited at different wavelengths. Crosscorrelation of both colors then allows for determining the binding behavior. If the lipids form a shell around the proteins, their co-diffusion yields a high crosscorrelation amplitude because the motion of both, lipids and proteins, is correlated. In case of free diffusion of proteins and lipids, the obtained crosscorrelation amplitude would be very low since the motion of lipids and proteins is uncorrelated. Moreover, since the crosscorrelation amplitude is inversely proportional to the particle number, 2cFCCS even allows for quantifying the amounts of co-diffusing lipids bound to the protein.

Aside from studying interactions between lipids and proteins, there is a great need to characterize the membranes themselves more accurately. One open question is 
the effect of membrane tension on protein diffusion. The membrane tension is a parameter which has been very challenging to measure thus far. Using our BLM system in combination with the 2fFCS setup, a new approach for tension measurements is possible, which involves incorporating fluorescently labeled lipids into the membrane and then moving a metal surface towards the bilayer. The proximity to the metal (gold) surface quenches the fluorescence within the membrane. The lifetime of the fluorescence dye is thus highly dependent on the distance to the metal surface. Due to this strong dependence, changes in the fluorescence lifetime are also directly linked to changes in membrane height. Fluorescence Lifetime Correlation Spectroscopy ( $(\overline{\text { FLCS }})$ then allows for calculating a correlation function between lifetime fluctuations and fluctuations in the membrane height.

The lifetime can be accurately measured using TCSPC. Since the changes in lifetime (i.e. the amount of quenching) is highly distance dependent, this method allows for determining the position of the membrane with respect to the metal surface very accurately. Membrane undulations can be monitored with about $200 \mathrm{~nm}$ spacial and about $10 \mathrm{~ns}$ temporal resolution. Using appropriate mathematical models, the membrane tension can be extracted from the measured membrane height correlation function.

In summary, combination of dual-focus FCS with Black Lipid Membranes yields highly precise diffusion coefficients in lipid bilayers, setting a new benchmark for membrane diffusion data as well as for determining lipid bilayer viscosity. The developed measurement and positioning methods have great potential for furhter investigation of previously challenging tasks, such as precisely determining lipidprotein interactions and accurately measuring membrane tensions. 


\section{Appendix}

\section{Determination of the Lipid Bilayer Thickness}

Precise determination of the bilayer thickness is challenging. Commonly used methods for measuring the membrane height are neutron and x-ray scattering ${ }^{62}$. In order to determine the bilayer thickness of the POPC/POPE membrane used in this study, we followed a new approach based on Förster Resonance Energy Transfer (FRET). Therefore, a BLM was formed using the Ionovation Bilayer Explorer and the perfusion channels above and below the pore were flushed each with a different dye. The dye concentrations were chosen so that a high concentration of acceptor dye (Alexa547) was present in the lower compartment, while a small concentration of donor dye (Atto488) was present in the upper compartment. The basic idea behind this approach is, that for every donor molecule, which is coming close to the membrane, an acceptor molecule is present on the other side of the bilayer providing a FRET partner at any given time. The experiment is illustrated in figure 42 .

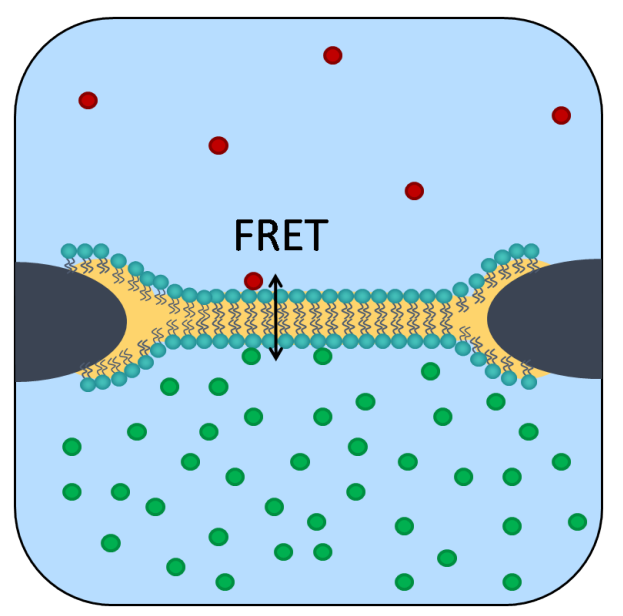

Figure 42: Determination of the membrane thickness using Förster Resonance Energy Transfer (FRET).

The FRET efficiency is determined by lifetime measuremetns. Therefore, the lifetime of the donor is determined in presence $\left(\tau_{\mathrm{DA}}\right)$ and in abscence of the acceptor $\left(\tau_{\mathrm{D}}\right)$. With the obtained lifetimes, the FRET transfer efficiency $E$ can be calculated according to

$$
E=1-\frac{\tau_{\mathrm{DA}}}{\tau_{\mathrm{D}}}
$$

The distance $r$ between the dyes, which corresponds to the bilayer thickness, is given 
by

$$
r=\sqrt[6]{\frac{R_{0}^{6}(1-E)}{E}}
$$

where $R_{0}$ denotes the Förster radius. The Förster radius is defined as the distance at which the FRET efficiency has decreased to $50 \%$ of its original value. $R_{0}$ for Atto488 (donor) and Alexa647 (acceptor) was calculated according to

$$
R_{0}^{6}=\frac{9000(\ln 10) \kappa^{2} Q_{D}}{128 \pi^{5} N n^{4}} \int_{0}^{\infty} F_{D}(\lambda) \epsilon_{A}(\lambda) \lambda^{4} d \lambda
$$

where $\kappa$ is the orientation factor which is assumed to be $\frac{2}{3}, Q_{D}$ is the quantum efficiency of the donor, $N$ denotes Avogadro's number and $n$ is the refractive index of the medium. $F_{D}(\lambda)$ denotes the normalized fluorescence intensity of the donor in the wavelength range $\lambda$ to $\lambda+\Delta \lambda . \epsilon_{A}(\lambda)$ is the acceptor's extinction coefficient at wavelength $\lambda$. For Atto488 and Alexa647, a Förster radius of $R_{0}=5.0813 \mathrm{~nm}$ was determined.

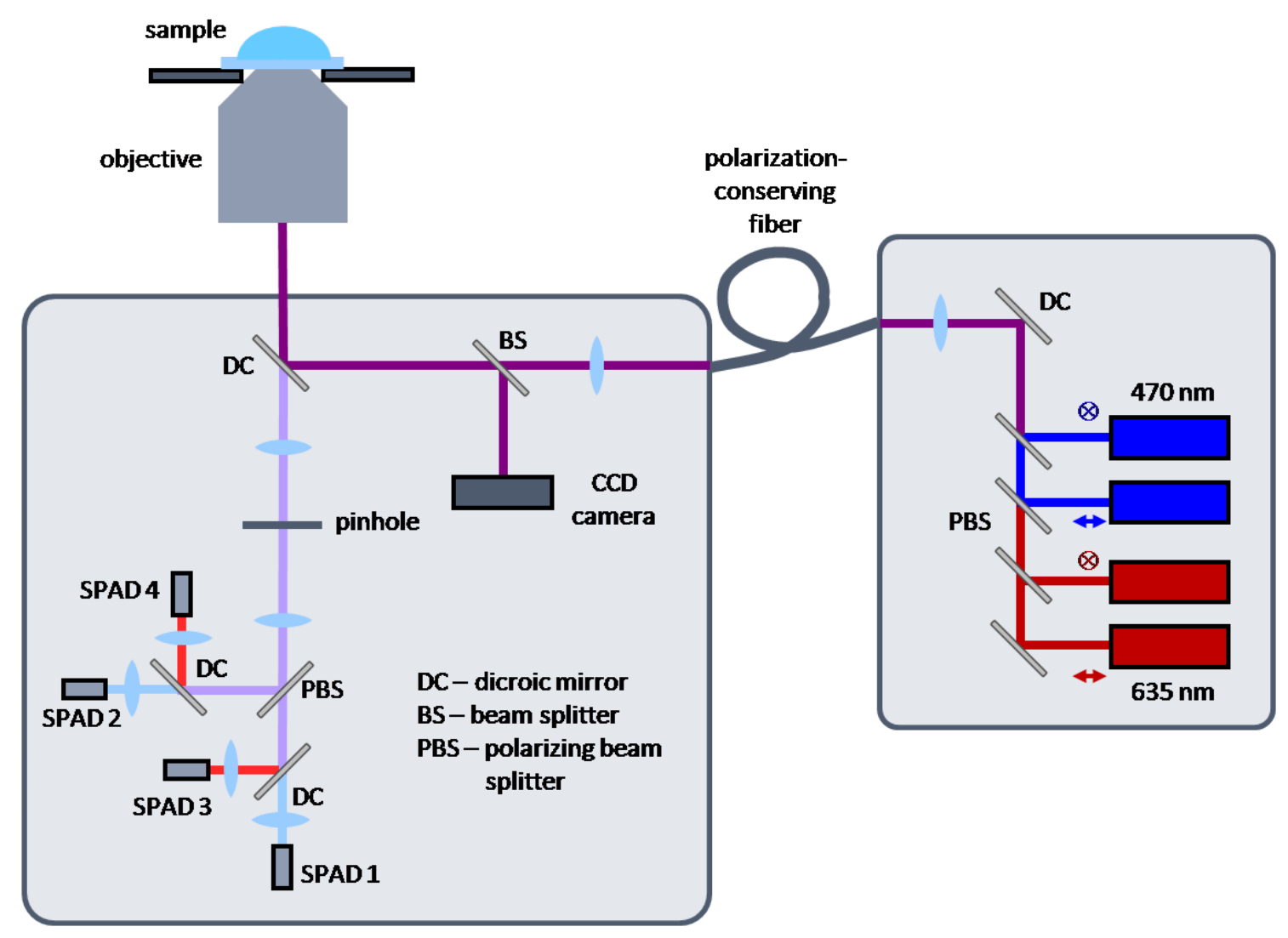

Figure 43: FRET measurement setup.

The used measurement setup is shown in figure 43. Two pulsed lasers of different colors (480 nm and $635 \mathrm{~nm}$ ) are used to excite the different dyes in donor only and 
acceptor only measurements. For FRET measurements, the sample is excited with the blue $(480 \mathrm{~nm})$ lasers while detection is done in the red channels.

In order to find the correct position for the lifetime measurements, an $x / z$-image of the pore was taken prior to the FRET measurements (figure 44). The membrane was visible upon addition of Atto488 but not when only Alexa647 was added. This indicates that the donor Atto488 attaches to the bilayer. For lifetime measurements, the foci were then positioned on the bilayer.

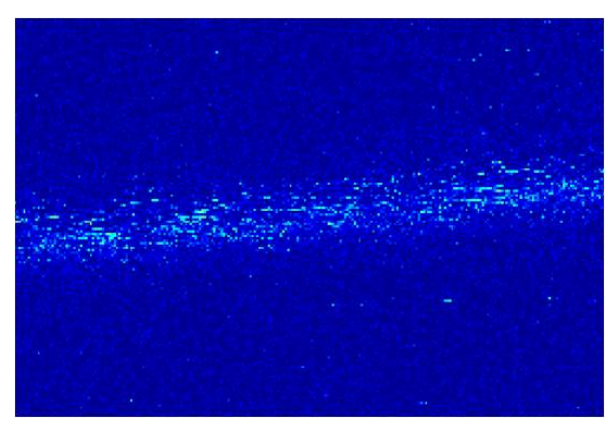

Figure 44: $x / z$-image of the membrane with Atto488 in the upper and Alexa647 in the lower compartment of the chip.

For the preliminary measurements performed so far, the fluorescent dyes were added directly into the chip instead of perfusing the BLM to avoid contamination of the tubing connected to the Bilayer Explorer. $10 \mu \mathrm{L} 30 \mathrm{nM}$ Atto488 maleimide and $30 \mu \mathrm{L} 1.5 \mu \mathrm{M}$ Alexa647 succinimidyl ester were added into the upper and the lower chamber, respectively. The $480 \mathrm{~nm}$ lasers were used for excitation and detection was done in the blue channel. The resulting lifetimes of the donor in presence $\left(\tau_{\mathrm{DA}}\right)$ and in abscence $\left(\tau_{\mathrm{D}}\right)$ of the acceptor in the lower chamber were measured and their distance was calculated using equation 23 .

Lifetime measurements were performed 10 times for 15 minutes each and a distance, i.e. membrane thickness, of $r=4.57 \pm 0.32 \mathrm{~nm}$ was obtained. This bilayer thickness is slightly larger but at least in the same range as the previously reported value of $r=3.8 \mathrm{~nm}^{59}$.

Further experiments need to be performed to validate the obtained preliminary result. Therefore, membranes consisting of lipids with different chain lengths need to be synthesized. FRET measurements should then reveal different $r$-values corresponding to different membrane thicknesses. 


\section{Bibliography}

[1] P. G. Saffman, M. Delbrück, "Brownian motion in biological membranes" Proceedings of the National Academy of Sciences of the United States of America (1975) 72(8), 3111-3113.

[2] Y. Gambin, R. Lopez-Esparza, M. Reffay, E. Sierecki, N. S. Gov, M. Genest, R. S. Hodges, W. Urbach, "Lateral mobility of proteins in liquid membranes revisited" Proceedings of the National Academy of Sciences of the United States of America (2006) 103(7), 2098-2102.

[3] A. Benda, M. Benes, V. Marecek, A. Lhotsky, W. T. Hermens, M. Hof, "How To Determine Diffusion Coefficients in Planar Phospholipid Systems by Confocal Fluorescence Correlation Spectroscopy" Langmuir (2003) 19(10), 4120-4126.

[4] E. P. Petrov, P. Schwille, "Translational diffusion in lipid membranes beyond the Saffman-Delbrück approximation" Biophysical Journal (2008) 94(5), L41L43.

[5] E. Gorter, F. Grendel, "On biomolecular layers of lipoids on the chromocytes of the blood" The Journal of Experimental Medicine (1925) 41(4), 439-443.

[6] J. F. Danielli, H. Davson, "A contribution to the theory of permeability of thin films" Journal of Cellular and Comparative Physiology (1935) 5(4), 495-508.

[7] S. J. Singer, Garth L. Nicolson, "The fluid mosaic model of the structure of cell membranes" Science (1972) 175(4023), 720-731.

[8] G. Caldieri, R. Buccione, "Aiming for invadopodia: organizing polarized delivery at sites of invasion" Trends in Cell Biology (2010) 20(2), 64-70.

[9] H.-X. Zhou, "Crowding Effects of Membrane Proteins" Journal of Physical Chemistry B (2009) 113(23), 7995-8005.

[10] A. A. Brian, H. M. McConnell, "Allogeneic stimulation of cytotoxic T cells by supported planar membranes" Proceedings of the National Academy of Sciences (1984) 81(19), 6159-6163. 
[11] E. Sackmann, "Supported membranes: scientific and Practical Applications" Science (1996) 271(5245), 43-48.

[12] T. Dertinger, I. von der Hocht, A. Benda, M. Hof, J. Enderlein, "Surface sticking and lateral diffusion of lipids in supported bilayers" Langmuir (2006) 22(22), 9339-9344.

[13] J. C. Stachowiak, D. L. Richmond, T. H. Li, A. P. Liu, S. H. Parekh, D. A. Fletcher, "Unilamellar vesicle formation and encapsulation by microfluidic jetting" Proceedings of the National Academy of Sciences (2008) 105(12), 46974702.

[14] P. Walde, K. Cosentino, H. Engel, P. Stano, "Giant Vesicles: preparations and applications" ChemBioChem (2010) 11(7), 848-865.

[15] M. I. Angelova, D. S. Dimitrov, "Liposome electroformation" Faraday Discussions of the Chemical Society (1986) 81(0), 303-311.

[16] K. Akashi, H. Miyata, H. Itoh, K. Kinosita Jr, "Preparation of giant liposomes in physiological conditions and their characterization under an optical microscope" Biophysical Journal (1996) 71(6), 3242-3250.

[17] N. Rodriguez, F. Pincet, S. Cribier, "Giant vesicles formed by gentle hydration and electroformation: a comparison by fluorescence microscopy" Colloids and Surfaces B: Biointerfaces (2005) 42(2), 125-130.

[18] L. R. Montes, A. Alonso, F. M. Goni, L. A. Bagatolli, "Giant Unilamellar Vesicles electroformed from native membranes and organic lipid mixtures under physiological conditions" Biophysical Journal (2007) 93(10), 3548-3554.

[19] D. L. Richmond, E. M. Schmid, S. Martens, J. C. Stachowiak, N. Liska, D. A. Fletcher, "Forming giant vesicles with controlled membrane composition, asymmetry, and contents" Proceedings of the National Academy of Sciences (2011) 108(23), 9431-9436.

[20] S. H. White, "Analysis of the torus surrounding planar lipid bilayer membranes" Biophysical Journal (1972) 12(4), 432-445.

[21] M. Montal, P. Mueller, "Formation of bimolecular membranes from lipid monolayers and a study of their electrical properties" Proceedings of the National Academy of Sciences (1972) 69(12), 3561-3566. 
[22] M. Kocun, T. D. Lazzara, C. Steinem, A. Janshoff, "Preparation of solvent-free, pore-spanning lipid bilayers: modeling the low tension of plasma membranes" Langmuir (2011) 27(12), 7672-7680.

[23] S. Ramadurai, A. Holt, V. Krasnikov, G. van den Bogaart, J. A. Killian, B. Poolman, "Lateral diffusion of membrane proteins" Journal of the American Chemical Society (2009) 131(35), 12650-12656.

[24] A. Naji, A. J. Levine, P. A. Pincus, "Corrections to the Saffman-Delbrück mobility for membrane bound proteins" Biophysical Journal (2007) 93(11), L49-L51.

[25] G. Guigas, M. Weiss, "Influence of hydrophobic mismatching on membrane protein diffusion" Biophysical Journal (2008) 95(3), L25-L27.

[26] C. Bräuchle, D.C. Lamb, J. Michaelis, Single Particle Tracking and Single Molecule Energy Transfer, Wiley VCH Weinheim (2009).

[27] D. Axelrod, D. E. Koppel, J. Schlessinger, E. Elson, W. W. Webb, "Mobility measurement by analysis of fluorescence photobleaching recovery kinetics" Biophysical Journal (1976) 16(9), 1055-1069.

[28] R. Rigler, E.L. Elson, Fluorescence Correlation Spectroscopy, Springer Berlin (2001).

[29] J. Ries, S. Chiantia, P. Schwille, "Accurate determination of membrane dynamics with line-scan FCS" Biophysical Journal (2009) 96(5), 1999-2008.

[30] T. Dertinger, V. Pacheco, I. von der Hocht, R. Hartmann, I. Gregor, J. Enderlein, "Two-Focus Fluorescence Correlation Spectroscopy: A New Tool for Accurate and Absolute Diffusion Measurements" ChemPhysChem (2007) 8(3), $333-333$.

[31] J. Ries, P. Schwille, "New concepts for fluorescence correlation spectroscopy on membranes" Physical Chemistry Chemical Physics (2008) 10(24), 3487-3497.

[32] R. Dutzler, E. B. Campbell, M. Cadene, B. T. Chait, R. MacKinnon, "X-ray structure of a $\mathrm{ClC}$ chloride channel at $3.0 \AA$ reveals the molecular basis of anion selectivity" Nature (2002) 415(6869), 287-294.

[33] H. I. Zgurskaya, H. Nikaido, "Bypassing the periplasm: reconstitution of the AcrAB multidrug efflux pump of Escherichia coli" Proceedings of the National Academy of Sciences (1999) 96(13), 7190-7195. 
[34] A. V. Pobbati, A. Stein, D. Fasshauer, "N- to C-terminal SNARE complex assembly promotes rapid membrane fusion" Science (2006) 313(5787), 673676.

[35] L. Heginbotham, M. LeMasurier, L. Kolmakova-Partensky, C. Miller, "Single streptomyces lividans $\mathrm{K}+$ Channels: functional asymmetries and sidedness of proton activation" The Journal of General Physiology (1999) 114(4), 551-560.

[36] F. I. Valiyaveetil, Y. Zhou, R. MacKinnon, "Lipids in the structure, folding, and function of the KcsA K+ channel" Biochemistry (2002) 41(35), 10771-10777.

[37] J. D. Faraldo-Gómez, E. Kutluay, V. Jogini, Y. Zhao, L. Heginbotham, B. Roux, "Mechanism of intracellular block of the KcsA K+ channel by tetrabutylammonium: insights from X-ray crystallography, electrophysiology and replicaexchange molecular dynamics simulations" Journal of Molecular Biology (2007) 365(3), 649-662.

[38] J. Lakowicz, Principles of Fluorescence Spectroscopy, Third Edition, Springer Science+Business Media New York (2006).

[39] B. K. Müller, E. Zaychikov, C. Bräuchle, D. C. Lamb, "Pulsed Interleaved Excitation" Biophysical Journal (2005) 89(5), 3508-3522.

[40] C. B. Müller, K. Weiß, W. Richtering, A. Loman, J. Enderlein, "Calibrating Differential Interference Contrast Microscopy with dual-focus Fluorescence Correlation Spectroscopy" Opt. Express (2008) 16(6), 4322-4329.

[41] T. Dertinger, A. Loman, B. Ewers, C. B. Müller, B. Krämer, J. Enderlein, "The optics and performance of dual-focus Fluorescence Correlation Spectroscopy" Opt. Express (2008) 16(19), 14353-14368.

[42] M. Wahl, I. Gregor, M. Patting, J. Enderlein, "Fast calculation of fluorescence correlation data with asynchronous time-correlated single-photon counting" Opt. Express (2003) 11(26), 3583-3591.

[43] D.V. O'Connor, D. Phillips, Time-correlated Single Photon Counting, Academic Press London (1984).

[44] W. Becker, Advanced Time-correlated Single Photon Counting Techniques, Springer Berlin (2005). 
[45] A.-S. Smith, S. F. Fenz, K. Sengupta, "Inferring spatial organization of bonds within adhesion clusters by exploiting fluctuations of soft interfaces" $E P L$ (Europhysics Letters) (2010) $\mathbf{8 9}(2), 28003$.

[46] R. A. Boeckmann, A. Hac, T. Heimburg, H. Grubmüller, "Effect of sodium chloride on a lipid bilayer" Biophysical Journal (2003) 85(3), 1647-1655.

[47] R. Vacha, S. W. I. Siu, M. Petrov, R. A. Boeckmann, J. Barucha-Kraszewska, P. Jurkiewicz, M. Hof, M. L. Berkowitz, P. Jungwirth, "Effects of alkali cations and halide anions on the DOPC lipid membrane" The Journal of Physical Chemistry A (2009) 113(26), 7235-7243.

[48] A. Filippov, G. Oraedd, G. Lindblom, "Effect of $\mathrm{NaCl}$ and $\mathrm{CaCl} 2$ on the lateral diffusion of zwitterionic and anionic lipids in bilayers" Chemistry and Physics of Lipids (2009) 159(2), 81-87.

[49] B. Alberts, A. Johnson, J. Lewis, M. Raff, K. Roberts, P. Walter, Molecular biology of the cell - 5th edition, Garland Science, Taylor \& Francis Group New York (2007).

[50] W. Jahnen-Dechent, M. Ketteler, "Magnesium basics" Clinical Kidney Journal (2012) 5(Suppl 1), i3-i14.

[51] T. A. Clarke, S.-C. Im, A. Bidwai, L. Waskell, "The role of the length and sequence of the linker domain of cytochrome B5 in stimulating cytochrome P450 2B4 catalysis" Journal of Biological Chemistry (2004) 279(35), 3680936818.

[52] R. Jahn, R. H. Scheller, "SNAREs - engines for membrane fusion" Nature Reviews Molecular Cell Biology (2006) 7(9), 631-643.

[53] A. T. Brunger, K. Weninger, M. Bowen, S. Chu, "Single-molecule studies of the neuronal SNARE fusion machinery" Annual Review of Biochemistry (2009) 78(1), 903-928.

[54] J. L. Robertson, L. Kolmakova-Partensky, C. Miller, "Design, function and structure of a monomeric ClC transporter" Nature (2010) 468(7325), 844-847.

[55] R. Dutzler, "A structural perspective on ClC channel and transporter function" FEBS Letters (2007) 581(15), 2839-2844. 
[56] M. A. Seeger, A. Schiefner, T. Eicher, F. Verrey, K. Diederichs, K. M. Pos, "Structural asymmetry of AcrB trimer suggests a peristaltic pump mechanism" Science (2006) 313(5791), 1295-1298.

[57] R. Blunck, H. McGuire, H. C. Hyde, F. Bezanilla, "Fluorescence detection of the movement of single KcsA subunits reveals cooperativity" Proceedings of the National Academy of Sciences (2008) 105(51), 20263-20268.

[58] B. D. Hughes, B. A. Pailthorpe, L. R. White, "The translational and rotational drag on a cylinder moving in a membrane" Journal of Fluid Mechanics (1981) 110, 349-372.

[59] R. Chen, D. Poger, A. E. Mark, "Effect of high pressure on fully hydrated DPPC and POPC bilayers" The Journal of Physical Chemistry B (2010) 115(5), 10381044.

[60] W. L. C. Vaz, F. Goodsaid-Zalduondo, K. Jacobson, "Lateral diffusion of lipids and proteins in bilayer membranes" FEBS Letters (1984) 174(2), 199-207.

[61] P. Cicuta, S. L. Keller, S. L. Veatch, "Diffusion of liquid domains in lipid bilayer membranes" The Journal of Physical Chemistry B (2007) 111(13), 3328-3331.

[62] Norbert Kucerka, Mu-Ping Nieh, John Katsaras, "Fluid phase lipid areas and bilayer thicknesses of commonly used phosphatidylcholines as a function of temperature" Biochimica et Biophysica Acta (BBA) - Biomembranes (2011) 1808(11), 2761-2771. 


\section{List of Figures}

1 Biological Membrane. . . . . . . . . . . . . . . . . 4

2 Chemical structure of cholesterol. . . . . . . . . . . . 5

3 Lipid Phases. . . . . . . . . . . . . . . . . 5

$4 \quad$ Schematic of Supported Lipid Bilayers. . . . . . . . . . . . . . . . 6

5 Giant Unilamellar Vesicles. . . . . . . . . . . . . . . . . . . 7

$6 \quad$ Formation of GUVs by lipid film hydration. $\ldots \ldots \ldots$. . . . . 7

7 Microfluidic jetting for GUV preparation. . . . . . . . . . . . 8

$8 \quad$ Black Lipid Membrane formation via painting. . . . . . . . . . . . . 10

$9 \quad$ Preparation of a solvent-free BLM from a lipid monolayer. . . . . . . 10

$10 \quad$ BLM formation upon spreading GUVs on a porous substrate. . . . . . 11

11 Saffman-Delbrück model. . . . . . . . . . . . . . . . . . . . . . . 13

12 Bilayer Slide used for BLM preparation. . . . . . . . . . . . . . 18

13 Home-built electroformation chamber for GUV synthesis. . . . . . . . 19

14 Cytochrome B5 monomer. . . . . . . . . . . . . . 20

15 KcsA tetramer. . . . . . . . . . . . . . . . . 20

16 EcClC dimer. . . . . . . . . . . . . . . . . . . . . . 21

17 AcrB trimer. . . . . . . . . . . . . . . . . . . 22

18 Jablonski diagram. . . . . . . . . . . . . . . . . . . 25

19 Dual-Focus FCS Setup. . . . . . . . . . . . . . . . . . 27

20 Generation of auto- and crosscorrelation functions. . . . . . . . . . . 29

21 Principle of time-correlated single photon counting (TCSPC). . . . 30

22 Classical TCSPC setup. . . . . . . . . . . . . . . . 31

23 Exemplary auto- and crosscorrelation functions. . . . . . . . . . 32

24 Connection between the radius of the laser beam which is coupled into the objective and the diameter of the resulting focus in sample

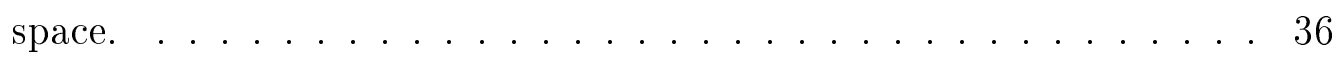

25 Modeled 2fFCS measurement of diffusion within a plane in an aberrationfree system. . . . . . . . . . . . . . . . . 37

26 Modeled 2fFCS measurement with aberrations due to refractive index mismatch. . . . . . . . . . . . . . . . . 39

27 Modeled 2fFCS measurement with aberrations due to cover slide thickness deviations. . . . . . . . . . . . . . . . . 40 40

$28 x / z$-Image of a POPC/POPE BLM . . . . . . . . . . . . . 45

$29 \quad z$-Scan across the membrane with 2fFCS. . . . . . . . . . . . . 46 
$30 \quad$ Autocorrelation and crosscorrelation curves for lipid diffusion of DPPE ${ }^{\text {Atto65 }}$ in a POPC/POPE BLM. . . . . . . . . . . . . . . . . 49

31 Influence of mono- and divalent cations on lipid diffusion. . . . . . . . 50

$32 x / z$-image of KcsA ${ }^{\text {Alexa647 }}$ in POPC/POPE BLM. . . . . . . . . . . . 55

33 2fFCS measurement of KesA ${ }^{\text {Alexa647 }}$ in surfactant solution. . . . . . . 55

34 2fFCS measurement of KesA ${ }^{\text {Alexa647 }}$ in solution below the surfactant's cmc. . . . . . . . . . . . . . . . 56

35 SDS PAGE of KcsA after purification without heating and with heating to $95{ }^{\circ} \mathrm{C}$ for 10 minutes. . . . . . . . . . . . . . 57

36 Autocorrelation and crosscorrelation curves of Kcs ${ }^{\text {Alexa647 }}$ in POPC/POPE BLM. . . . . . . . . . . . . . . 58

37 Primary structures of Syntaxin-1, SNAP-23 and Synaptobrevin-2. . . 59

38 X-ray crystal structure of the core of the neuronal SNARE complex. . 59

39 Model of two SNARE complexes in the trans state, which dock a liposome to a SLB in vitro . . . . . . . . . . . . . . . . . . . . 60

40 Electrophysiology measurements of KcsA in POPC/POPE/DOPG bilayer. . . . . . . . . . . . . . . . 6 6 . . . . . . . 64

41 Saffman-Delbrück vs. Stokes-Einstein model. . . . . . . . . . . . . . . 64

42 Determination of membrane thickness with FRET . . . . . . . . . 73

43 FRET measurement setup. . . . . . . . . . . . . . . . . . . 74

$44 x / z$-image of the membrane with Atto488 in the upper and Alexa647 in the lower compartment of the chip. . . . . . . . . . . . 75 


\section{Acronyms}

2cFCCS Dual-color Fluorescence Crosscorrelation Spectroscopy

2fFCS Dual-focus Fluorescence Correlation Spectroscopy

ACFs autocorrelation functions

ADC analog-to-digital converter

AMP amplifier

APDs avalanche photodiodes

Biotinyl-PE 1,2-dioleoyl-sn-glycero-3-phosphoethanol-amine-N-(biotinyl) sodium salt

BLMs Black Lipid Membranes

CCFs Crosscorrelation Functions

CFD Constant Fraction Discriminator

cmc critical micelle concentration

CytB5 Cytochrome-B5

DM Decyl maltoside

DOPG 1,2-di-(9Z-octadecenoyl)-sn-glycero-3-phospho-(1'-rac-glycerol) sodium salt

DPPE ${ }^{\text {Atto655 1,2-dihexadecanoyl-sn-glycero-3-phosphoethanolamine }}$

E.coli Escherichia coli

FCS Fluorescence Correlation Spectroscopy

FRAP Fluorescence Recovery After Photobleaching

FWHM Full Width Half Maximum

FLCS Fluorescence Lifetime Correlation Spectroscopy

FRET Förster Resonance Energy Transfer 
GUVs Giant Unilamellar Vesicles

HEPES 4-(2-hydroxyethyl)-1-piperazineethanesulfonic acid

HPW Hughes, Pailthorpe, and White

IPTG isopropyl $\beta$-D-1-thiogalactopyranoside

LUVs Large Unilamellar Vesicles

MDF Molecule Detection Function

MLVs Multilamellar Vesicles

MWCO molecular weight cut-off

N.A. numerical aperture

NSF N-ethylmaleimide-sensitive factor

Ni-NTA Nickel nitrioltriacetic acid

OG Octyl glycoside

PBS phosphate buffered saline

PI protease inhibitor

PIE $\quad$ pulsed interleaved excitation

PIPES piperazine-N,N'-bis(2-ethanesulfonic acid)

PMSF phenylmethylsulfonyl fluoride

PN Particle Number

POPC 1-palmitoyl-2-oleoyl-sn-glycero-3-phosphocholine

POPE 1-palmitoyl-2-oleoyl-sn-glycero-3-phosphoethanolamine

SDS PAGE sodium dodecyl sulfate polyacrylamide gel electrophoresis

SLBs Supported Lipid Bilayers

SNARE soluble N-ethylmaleimide-sensitive-factor attachment receptor

SPADs $\quad$ Single Photon Avalanche Diodes 
SPT Single Particle Tracking

SUVs Small Unilamellar Vesicles

Syb Synaptobrevin-2

TAC time-to-amplitude converter

TBA tetra butyl ammonium

TCSPC time-correlated single photon counting 


\section{Acknowledgments}

Jörg Enderlein möchte ich dafür danken, dass ich meine Doktorarbeit in seiner Gruppe anfertigen durfte. Deine Tür war immer offen, sogar als ich noch nicht in Göttingen angefangen hatte. Vielen Dank für all deine Hilfe, deine Anregungen und dafür, dass du dir immer Zeit für Erklärungen und Gespräche genommen hast. Ich freue mich sehr, dass ich so viel von dir lernen durfte.

Sarah Köster \& Andreas Janshoff, meinem Thesis Committee, möchte ich danken für die vielen hilfreichen Diskussionen, Vorschläge und die große Unterstützung.

Geert van den Bogaart, you always took time to discuss my project with me. Thank you for all the helpful advice and suggestions. You always had another good idea in mind, patiently explained the "biology wodoo" to me and provided me with the SNAREs. I appreciate your help very much.

I would also like to thank Fabio Villardi \& Blanche Schwappach for providing me with generous amounts of Cytochrome-B5 and for answering so many of my questions about it.

Andreas Neef danke ich besonders für seine Hilfe bei den ElektrophysiologieMessungen.

Bei Raimund Dutzler bedanke ich mich für seine vielen hilfreiche Anregungen und Diskussionen zum Thema EcClC.

Außerdem möchte ich mich bei der gesamten Arbeitsgruppe für die gute Atmosphäre und Hilfsbereitschaft bedanken, speziell bei:

Ingo Gregor für seine Hilfe mit dem 2fFCS-Aufbau und viele hilfreiche Anregungen und Erklärungen während der gesamten Zeit.

Stefanie Kramer dafür, dass sie mir die Proteinaufreinigung gezeigt und viele Fragen zur Arbeit in einem Biolabor beantwortet hat. Außerdem vielen Dank, dass du immer schnell und zuverlässig alles bestellt hast, was wir brauchten.

Mira Prior, LaTeX-Queen, Enten-Mama und Hotelzimmmer-Mitbewohnerin. Danke, dass ich immer auf dich zählen konnte und du immer für mich da warst. Danke für all deine Hilfe, ob LaTeX, Physik oder Lebensfragen und dafür, dass ich dich mit all meinen Sorgen und Nöten über Membranproteine und Lipide nerven durfte. Du hast dir geduldig auch zum zwanzigsten Mal meine Vorträge am Abend vorher angehört, was ich dir hoch anrechne (ich glaube, du kannst mittlerweile mitsprechen...). Vielen Dank für's Beruhigen, gute Zureden und für deine Versorgung mit Kaugum- 
mis, Kokosmakronen und Seifenblasen besonders in schlechten Zeiten. Du bist mein ausgelagertes gutes Gewissen und mein Fels in der Brandung.

Phillip Kroehn, Mitstreiter, Wegbegleiter, "Sternzeichen Schlingel". Ich danke dir für viele Überraschungen in und um meinen Schreibtisch. Deinen Sprung aus der Kiste und den Wecker in meinem Regal werde ich so schnell nicht vergessen. Du hast mich aufgeheitert, wenn es mir schlecht ging und hattest immer den passenden Kommentar parat. Deine (überwiegend schlechten ;-)) Witze werde ich schmerzlich vermissen. Du hast mir während der letzten Jahre oft geholfen, sei es mit einem offenen Ohr oder deiner überwältigenden Fähigkeit nützliche Dinge aus für mich völlig abstrusen Kombinationen zu bauen. Danke!

Christoph Pieper, lange Zeit der dritte im (Büro-)Bunde. Du hast mir vieles zu FCS und Physik erklärt und geduldig die Dekorationsversuche unseres Zimmers ertragen. Danke, dass du deine ThorLabs Snack-Boxen mit uns geteilt und uns gezeigt hast, wie akkurat gefeilte Fingernägel aussehen.

Anja Huss, Spitzenkonditorin und Führerscheinzwilling. Dir danke ich für deine vielen ausführlichen Erklärungen zu STORM und SOFI, viele lustige Mittagspausen, deine wunderbaren Backkünste und dafür, dass du verstehst was "Tembo, seine Arme weit" bedeutet.

Weiterhin möchte ich mich bei dir, Bernd Müller, dafür bedanken, dass du mich nach Göttingen geholt hast. Du hast mich schon während meinem Studium gefördert und mich mit 2fFCS bekannt gemacht. Danke für deine geduldigen Erklärungen, dafür, dass deine Tür immer offen war und besonders dafür, dass du mir den Einstieg in Göttingen sehr erleichtert hast.

Harald Reuter, dir danke ich dafür, dass du immer an mich geglaubt und mich ermutigt hast. Danke für deinen Rückhalt, dein Vertrauen und dafür, dass du bei allen Erfolgen, aber auch bei kleinen und großen Problemen immer da warst.

Meinen Eltern möchte ich danken, ohne deren Rückhalt und Untersützung mein Studium und meine Promotion nicht möglich gewesen wären. Ihr seid immer für mich da gewesen, habt mitgefiebert und mitgelitten, Daumen gedrückt und Kerzen im Kölner Dom angezündet. Ich möchte mich außerdem bei meiner gesamten Familie bedanken, speziell bei Anna Bischof, Lieblingscousine und Schwester-Ersatz, weil dir ein Vorbild zu sein mich in vielen Fällen zu Höchstleistungen angetrieben hat. 


\section{Education}

August 2010 - PhD in Biophysics, Georg-August University Göttingen August 2013

January 2010 Diploma in Physical Chemistry, RWTH Aachen University

- July 2010

August 2006 Vordiplom in Chemistry, RWTH Aachen University

October 2004 Chemistry studies at RWTH Aachen University

- July 2010

August 1995 High School - Gutenberg Gymnasium Bergheim

- June 2004

\section{Research Experience}

August 2010 - PhD Thesis in Biophysics, Georg-August University Göttingen

August 2013 Title: „Quantifying the diffusion of membrane proteins and peptides“ Supervisor: Prof. Jörg Enderlein

January 2010 Diploma Thesis in Physical Chemistry, RWTH Aachen University

- July 2010 Title: „Probing the interactions of (bio-)polymers with PNiPAM-based micro- and hydrogels by means of $2 \mathrm{fFCS}$ “

Supervisor: Prof. Walter Richtering

July 2008 - Research Internship in Organic Chemistry, UC Berkeley

December Title: "Towards the total synthesis of triscyclopropabenzene"

2008 Supervisor: Prof. Peter Vollhardt

April 2008 - Research Internship in Chemical Engineering, University of Delaware

June 2008 Title: „Self-assembly and rheology of worm-like micelle-colloid mixtures“ Supervisor: Prof. Norman J. Wagner

August 2007 - Student researcher in Physical Chemistry, RWTH Aachen University

October 2007 Implementation and calibration of a Dual-Focus Fluorescence Correlation Spectroscopy setup

Supervisor: Prof. Walter Richtering 


\section{Publications}

K. Weiß, A. Neef, S. Kramer, Q. Van, I. Gregor, J. Enderlein, "Quantifying the Diffusion of Membrane Proteins and Peptides in Black Lipid Membranes with 2-Focus Fluorescence Correlation Spectroscopy", Biophysical Journal, 2013, 105, 455 - 462.

C. Pieper, K. Weiß, I. Gregor, J. Enderlein, "Dual-focus Fluorescence Correlation Spectroscopy", Methods in Enzymology, 2013, 518, 175 - 204.

K. Weiß, J. Enderlein, "Lipid diffusion within Black Lipid Membranes measured with Dual-Focus Fluorescence Correlation Spectroscopy", ChemPhysChem, 2012, 13, 990 1000.

C.B. Müller, K. Weiß, A. Loman, J. Enderlein, W. Richtering, "Remote temperature measurements in femto-liter volumes using dual-Focus Fluorescence Correlation Spectroscopy", Lab on a Chip, 2009, 9, 1248 - 1253.

C.B. Müller, K. Weiß, W. Richtering, A. Loman, J. Enderlein, "Calibrating Differential Interference Contrast Microscopy with Dual-Focus Fluorescence Correlation Spectroscopy", Optics Express, 2008, 16, 4322 - 4329.

\section{Conferences}

\section{Talks}

"Quantifying the Diffusion of Membrane Proteins and Peptides“, DPG Spring Meeting of the Condensed Matter Section, Regensburg, 2013.

"Quantifying the Diffusion of Membrane Proteins and Peptides", 17th International Workshop on Single Molecule Spectroscopy, Berlin, 2011.

„Lipid and Protein Diffiusion in Black Lipid Membranes“, SFB 803 Symposium, Göttingen, 2011

„Investigation of interactions between Microgels and Polyelectrolytes by means of 2fFCS“, 16th International Workshop on Single Molecule Spectroscopy Berlin, 2010.

Posters

K. Weiß, J. Enderlein, „Quantifying Lipid and Protein Diffusion in Black Lipid Membranes by means of 2fFCS“, 18th International Workshop on Single Molecule Spectroscopy, Berlin, September 2012. 
K. Weiß, J. Enderlein, „Quantifying Lipid Diffusion in Black Lipid Membranes“, 56th Annual Meeting of the Biophysical Society, San Diego, CA, USA, 2012.

K. Weiß, C.B. Müller, J. Enderlein, W. Richtering, „Probing Colloids with 2fFCS“, Gordon Research Conference, Ventura, CA, USA, 2010.

K. Weiß, C.B. Müller, J. Enderlein, W. Richtering, „Probing Colloids with 2fFCS“, ACS Colloids Conference, Rayleigh, NC, USA, 2008. 